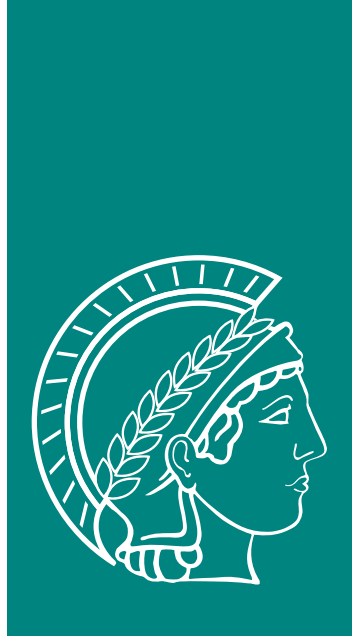

\title{
Structure and Dynamics of Ionic Liquid/Electrode Interfaces
}

\author{
Peter Reichert \\ Mainz 2014
}

Max-Planck-Institut für Polymerforschung Mainz Johannes Gutenberg-Universität Mainz 



\title{
Structure and Dynamics of Ionic Liquid/Electrode Interfaces
}

\author{
Dissertation \\ zur Erlangung des Grades \\ "Doktor der Naturwissenschaften" \\ im Promotionsfach Physik
}

Fachbereich Physik, Mathematik und Informatik der Johannes Gutenberg-Universität in Mainz

Peter Reichert geb. in Mainz

Mainz, April 2014 
Tag der mündlichen Prüfung:

Erster Berichterstatter:

Zweite Berichterstatterin:
25.06.2014

[In der elektronischen Fassung aus Datenschutzgründen entfernt] [In der elektronischen Fassung aus Datenschutzgründen entfernt] 


\section{Zusammenfassung}

$\mathrm{Zu}$ Beginn des zwanzigsten Jahrhunderts entwickelten Gouy, Chapman und Stern eine Theorie der elektrochemischen Doppelschicht in verdünnten Elektrolytlösungen. Diese erlaubt die Berechnung der Kapazität einer Elektrolyt/Elektroden-Grenzfläche aus der Ionenverteilung nahe der Elektrode. Nach einem Jahrhundert intensiver Forschung konnten entscheidende Fortschritte im Verständnis der Elektrolyt/Elektroden-Grenzfläche erzielt werden. Allerdings wirft die genaue molekulare Grenzflächenstruktur und deren Zusammenhang mit dem angelegten Elektrodenpotential gegenwärtig noch immer Fragen auf. Insbesondere ionische Flüssigkeiten, eine neue Klasse von lösungsmittelfreien Elektrolyten, können durch klassische Modelle nicht beschrieben werden. Jüngste Molekulardynamiksimulationen und phänomenologische Theorien haben versucht die Kapazität der ionischen Flüssigkeit/Elektroden-Grenzfläche durch die molekulare Struktur und die Dynamik der ionischen Flüssigkeit nahe der Elektrode zu erklären. Allerdings sind experimentelle Belege rar.

In der vorgelegten Arbeit wurde die Ionenverteilung einer ionischen Flüssigkeit nahe der Elektrodengrenzfläche und deren Reaktion auf ein angelegtes Elektrodenpotential mit submolekularer Auflösung untersucht. Hierfür wurde eine Probenzelle entworfen, welche sowohl für in situ Röntgenreflektometrie unter Potentialkontrolle als auch für elektrochemische Impedanzspektroskopiemessungen geeignet ist. Die Kombination von struktureller und elektrochemischer Information ermöglicht einen tiefen Einblick in das Wesen der elektrochemischen Doppelschicht. So wurden oszillierende Ladungsdichteprofile bestehend aus alternierenden Kation- und Anion-angereicherten Lagen nachgewiesen, welche sowohl bei anodischem als auch kathodischem Potential vorhanden sind. Diese Grenzflächenstruktur ist eine direkte Folge von intrinsischen Ladungskorrelationen, welche auch die Volumenstruktur prägen und als charakteristische Röntgenstrukturpeaks beobachtet wurden. Zur Beschreibung dieser Korrelationen wurden bereits existierende physikalisch motivierte Modelle erweitert und durch unabhängige Messungen bestätigt.

Die induzierte Relaxationsdynamik der Grenzflächenstruktur nach einem Potentialsprung wurde mittels zeitaufgelöster Röntgenreflektometrie mit einer Zeitauflösung unterhalb einer Millisekunde untersucht. Die gemessenen Relaxationszeiten stimmen mit Impedanzspektroskopieergebnissen überein. So konnten drei getrennte Prozesse auf drei unterschiedlichen Zeitskalen identifiziert werden. Der Ionentransport senkrecht zur Grenzfläche geschieht im Bereich einer Millisekunde. Ein zweiter Prozess, auf einer Zeitskala von hundert Millisekunden, konnte einer molekularen Umorientierung an der 
Grenzfläche zugeordnet werden. Schließlich wurde ein langsamer Prozess auf Minutenskala beobachtet, der möglicherweise einer lateralen Ordnung der adsorbierten Ionenlage zugeschrieben werden kann. 


\section{Abstract}

In the early 20th century, Gouy, Chapman, and Stern developed a theory to describe the capacitance and the spatial ion distribution of diluted electrolytes near an electrode. After a century of research, considerable progress has been made in the understanding of the electrolyte/electrode interface. However, its molecular-scale structure and its variation with an applied potential is still under debate. In particular for room-temperature ionic liquids, a new class of solventless electrolytes, the classical theories for the electrical double layer are not applicable. Recently, molecular dynamics simulations and phenomenological theories have attempted to explain the capacitance of the ionic liquid/electrode interface with the molecular-scale structure and dynamics of the ionic liquid near the electrode. However, experimental evidence is very limited.

In the presented study, the ion distribution of an ionic liquid near an electrode and its response to applied potentials was examined with sub-molecular resolution. For this purpose, a new sample chamber was constructed, allowing in situ high energy Xray reflectivity experiments under potential control, as well as impedance spectroscopy measurements. The combination of structural information and electrochmical data provided a comprehensive picture of the electric double layer in ionic liquids. Oscillatory charge density profiles were found, consisting of alternating anion- and cation-enriched layers at both, cathodic and anodic, potentials. This structure was shown to arise from the same ion-ion correlations dominating the liquid bulk structure that were observed as a distinct X-ray diffraction peak. Therefore, existing physically motivated models were refined and verified by comparison with independent measurements.

The relaxation dynamics of the interfacial structure upon potential variation were studied by time resolved X-ray reflectivity experiments with sub-millisecond resolution. The observed relaxation times during charging/discharging are consistent with the impedance spectroscopy data revealing three processes of vastly different characteristic time-scales. Initially, the ion transport normal to the interface happens on a millisecondscale. Another 100-millisecond-scale process is associated with molecular reorientation of electrode-adsorbed cations. Further, a minute-scale relaxation was observed, which is tentatively assigned to lateral ordering within the first layer. 


\section{Contents}

Zusammenfassung

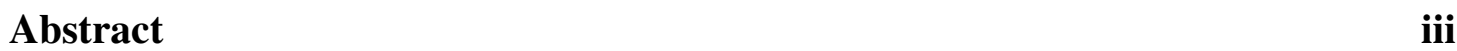

I IONIC LIQUIDS

1 Introduction $\quad 3$

2 Theory of liquids 5

2.1 Inter-particle forces . . . . . . . . . . . . . . 5

2.2 Total correlation function . . . . . . . . . . . . . . 6

2.3 Ion-ion correlations at the interface . . . . . . . . . . . 9

3 The electric double layer $\quad 11$

3.1 Poisson-Boltzmann theory of the diffuse double layer . . . . . . . . . . 11

3.2 Diffuse double layers in ionic liquids . . . . . . . . . . . . . . 14

3.3 Charging/discharging dynamics . . . . . . . . . . . . . 17

4 Structure and dynamics of ionic liquids

4.1 Bulk properties . . . . . . . . . . . . . . . 21

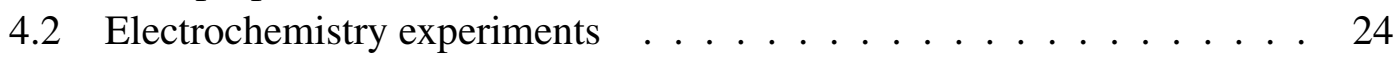

4.3 Interfacial structure $\ldots \ldots \ldots 26$

II MATERIALS AND METHODS 31

5 Material properties $\quad 33$

5.1 Ionic liquids . . . . . . . . . . . . . . . . . 33

5.2 Electrode materials . . . . . . . . . . . . . . . 38

6 X-ray scattering 43

6.1 The liquid structure factor . . . . . . . . . . . . . . 45

6.2 Teubner-Strey model . . . . . . . . . . . . . . 50 
6.3 X-ray reflectivity . . . . . . . . . . . . . . . 54

6.4 Modified distorted crystal model . . . . . . . . . . . . 56

7 Impedance spectroscopy $\quad 63$

7.1 Debye process . . . . . . . . . . . . . . . . 64

7.2 Electrode polarization . . . . . . . . . . . . . . . . 65

7.3 Capacitance model . . . . . . . . . . . . . . 66

7.4 Equivalent circuit . . . . . . . . . . . . . . . . . 68

8 Experimental setup and data aquisition $\quad \mathbf{7 1}$

8.1 Electrochemical sample cell for in situ X-ray reflectivity . . . . . . . 71

8.2 High energy X-ray reflectivity . . . . . . . . . . . . . 76

8.3 Impedance spectroscopy . . . . . . . . . . . . . . . . 82

8.4 Bulk X-ray diffraction . . . . . . . . . . . . . . . 82

III RESULTS AND DISCUSSION 85

9 The $[\text { bmpy }]^{+}[\text {FAP }]^{-} /$electrode interface $\quad 87$

9.1 Impedance spectroscopy results . . . . . . . . . . . . . . . . . . . 87

9.2 Interfacial structure . . . . . . . . . . . . . . . . 90

9.3 Dynamics . . . . . . . . . . . . . . . . . 93

10 The $[\text { tba }]^{+}[\text {FAP }]^{-} /$electrode interface $\quad 97$

10.1 Interfacial structure . . . . . . . . . . . . . . . . . . . 97

10.2 Dynamics . . . . . . . . . . . . . . . . . 100

$\begin{array}{ll}11 \text { Conclusions } & 101\end{array}$

$\begin{array}{ll}12 & \text { Other results and outlook } \\ \end{array}$

$\begin{array}{lr}\text { A Abbreviations and acronyms } & 105\end{array}$

$\begin{array}{lll}\text { B Symbols used in equations } & 107\end{array}$

$\begin{array}{ll}\text { Bibliography } & 111\end{array}$

$\begin{array}{ll}\text { Danksagung } & 127\end{array}$ 


\section{Part I}

\section{IONIC LIQUIDS}





\section{Chapter 1}

\section{Introduction}

Ionic liquids (ILs) are salts with melting points below $100{ }^{\circ} \mathrm{C}$. Their history ${ }^{1}$ dates back to 1914 when Walden reported the discovery of ethylammonium nitrate, a salt with a melting point of $12^{\circ} \mathrm{C}$ [167]. This temperature is remarkably low compared to the melting temperatures of other salts like sodium chloride $\left(801^{\circ} \mathrm{C}\right) .^{2}$ Recently, the topic draw increasing attention, after the first synthesis of air and water stable ILs was reported in 1992 [172]. Up to the present day a vast number of ILs has been synthesized. They commonly consist of large, asymmetric, molecular ions (figure 1.1). By altering the chemical structure of anion and cation, compounds with low vapor pressure, low melting point, high thermal stability, wide electrochemical window, and high conductivity have been synthesized. These superior electrochemical properties give rise to a wide range of possible applications of ILs as electrolytes in environmentally safe and energy efficient electrochemical processes, e.g. electroplating, batteries, organic solar cells, fuel cells and supercapacitors. In these applications the electrolyte/electrode interface plays a crucial role. Therefore, a detailed knowledge of the spatial arrangement of ILs near the liquid/solid interface is most desirable.

This thesis aims to elucidate the molecular-scale structure of the IL/electrode interface and its response to applied electrode potentials. It is divided into three parts. The first part IONIC LIQUIDS comprises an overview on the field of ionic liquids and the theoretic background necessary to understand the properties of ionic liquids. The fundamental thermodynamics of simple liquids are presented in Chapter 2. It is outlined how the inter-molecular structure arises from two-particle forces. Further, general asymptotic correlations are presented in bulk and at the interface. The interface between a charged wall and an electrolyte is discussed in Chapter 3. Here, models are presented that link the capacitance to the ion arrangement at the interface. The dynamic response of the ion arrangement upon electrode potential variation is shown to be dependent on the local charge concentration profile at the interface and on the macroscopic ion diffu-

\footnotetext{
${ }^{1}$ For a comprehensive overview on the history of ionic liquids, the interested reader is referred to $[170,171]$.

${ }^{2}$ Also salt mixtures can have a very low meting points. For instance, the eutectic melt of sodium chloride and aluminum chloride has a melting temperature of only $107^{\circ} \mathrm{C}$.
} 


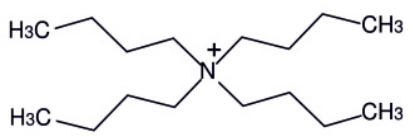

(a)

$[\mathrm{tba}]^{+}$

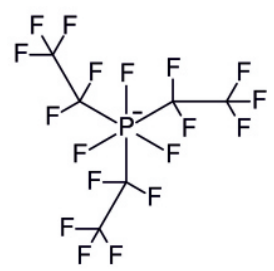

(b)

[FAP]

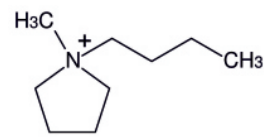

$[\mathrm{bmpy}]^{+}$

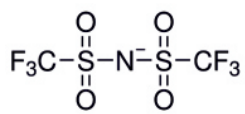

$[\mathrm{TFSI}]$

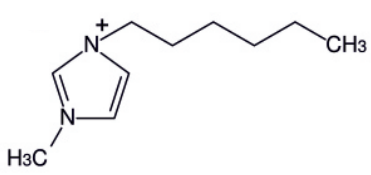

$[\mathrm{hmim}]^{+}$

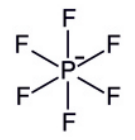

$\left[\mathrm{PF}_{6}\right]$

Figure 1.1: A selection of cations (a) and anions (b) used in ILs: tetrabutylammonium [tba $]^{+}$, 1-butyl-1-methylpyrrolidinium $[\text {bmpy }]^{+}, \quad$ 1-hexyl-3methylimidazolium $[\mathrm{hmim}]^{+}$, tris(pentafluoroethyl)trifluorophospate $[\mathrm{FAP}]^{-}$, bis(trifluoromethylsulfonyl)imide $[\mathrm{TFSI}]^{-}$, hexafluorophosphate $\left[\mathrm{PF}_{6}\right]^{-}$.

sion between the electrodes. Further, the impact of steric effects and ion-ion correlations is discussed. After presenting the theoretical basis, an overview on the recent experimental findings is given in Chapter 4. A common observation is that ILs exhibit strong ion-ion correlations in bulk and at the interface. They strongly effect charge transport and electrochemical properties.

The second part MATERIALS AND METHODS begins with a presentation of the ILs investigated in this work. In Chapter 5 the physical properties of the ILs $[\mathrm{bmpy}]^{+}[\mathrm{FAP}]^{-}$and $[\mathrm{tba}]^{+}[\mathrm{FAP}]^{-}$are presented, followed by a discussion on electrode materials. Chapter 6 outlines the fundamentals of X-ray scattering. This technique is used to extract structural information from bulk liquid and the liquid/solid interface. Phenomenological models are developed describing long range ion-ion correlations in bulk and at the interface. Chapter $\mathbf{7}$ gives an introduction in electrochemical methods and the general phenomenological description of relaxation processes. In Chapter 8 the experimental setup is described which was used to perform the in situ X-ray diffraction and impedance spectroscopy experiments.

The third part RESULTS AND DISCUSSION is divided into two chapters each comprising the results of an IL/electrode system under investigation. In Chapter 9 the results of impedance spectroscopy, X-ray reflectivity and bulk X-ray diffraction are combined, giving a comprehensive picture of the $[\mathrm{bmpy}]^{+}[\mathrm{FAP}]^{-} /$electrode interface. The content of this chapter is the basis of a manuscript, which will be published soon [121]. The results of the second IL [tba] ${ }^{+}[\mathrm{FAP}]^{-}$are discussed in Chapter 10 confirming the findings of the previous chapter. Finally, conclusions of thesis are drawn in Chapter 11 and its relevance to related fields of research and applications is discussed. 


\section{Chapter 2}

\section{Theory of liquids}

ILs can be described in the thermodynamic framework of classical liquids ${ }^{1}$. The liquid state of matter is distinguished from the crystalline solid state by a lack of long-range order. However, the great importance of collision processes leads to short-range correlations, which are not present in the gaseous state ${ }^{2}$. The comparable values of potential and kinetic energy result in a microscopic structure balancing inter-particle forces and thermal excitation. The following sections outline how the collective correlations arise from two-particle forces. Further, the asymptotic decay of the correlations is discussed both in bulk and at the interface. A comprehensive overview on the general thermodynamics of liquids can be found in literature [44, 62].

\subsection{Inter-particle forces}

In ILs, the inter-particle forces arise from different contributions to the potential $\phi_{\alpha \beta}(r)$ between ions of species $\alpha$ and $\beta$ separated by a distance $r$

$$
\phi_{\alpha \beta}(r)=\phi_{\alpha \beta}^{\mathrm{sr}}(r)+\frac{Z_{\alpha} Z_{\beta} e^{2}}{4 \pi \varepsilon_{r} \varepsilon_{0} r}-\frac{A_{\alpha \beta}}{r^{6}} .
$$

The short-range potential $\phi_{\alpha \beta}^{\mathrm{sr}}(r)$ results from the Pauli repulsion of overlapping electron orbitals and is approximately described by a term proportional to $r^{-12}$ as in the famous Lennard-Jones potential. The second term describes the Coulomb interaction between the ions of charge $Z_{\alpha, \beta}$ leading to repulsion for like ions and attraction for ions of different charge. In spite of its $r^{-1}$ dependence, the Coulomb interaction in ILs is effectively short-ranged due to screening by counter ions. The third, attractive dispersion term originates from several effects, which are all of $r^{-6}$ dependence. Namely, these are the Keesom interaction between permanent dipoles, the Debye interaction between induced dipoles, and the London dispersion interaction stemming from quantum

\footnotetext{
${ }^{1}$ For classical liquids, the de Broglie wavelength of a liquid particle is smaller than its diameter. Thus, quantum effects, e.g. giving rise to super-fluidity, can be neglected.

${ }^{2}$ below the critical point
} 
mechanical fluctuations of the electron cloud [22]. These interactions are generally summarized as van der Waals interaction with an overall amplitude of $A_{\alpha \beta}$. The potential (2.1) well describes the interaction in atomic molten salts like sodium chloride [127]. For ILs, which consist of large organic molecules, the inter-molecular potential is much more complex. One has to sum the contributions of all atomic sites in each molecule and include steric effects stemming from the intra-molecular structure [76]. Nevertheless, by applying the simple potential (2.1) it is possible to reproduce physical properties of ILs similar to results from MD simulations based on more complex inter particle forces [47, 103].

\subsection{Total correlation function}

The microscopic structure of liquids results from correlations balancing inter-particle forces and thermal excitation. A measure of this structure is the radial distribution function $g(r)$ (cf. section 6.1). It gives the average probability of finding a liquid particle at a distance $r$ from a test particle. The average is taken over an ensemble of micro states of the liquid. In the following it is more convenient to speak of correlations than distributions. Thus, the total correlation function is defined

$$
h(r)=g(r)-1 .
$$

The two functions differ in their asymptotic behavior. For large distances the probability of finding a second particle becomes unity, i.e. $g(r \rightarrow \infty)=1$, while the particles become more and more uncorrelated, i.e. $h(r \rightarrow \infty)=0$. The total correlation function $h(r)$ is accessible via neutron and X-ray scattering experiments. The scattered intensity is equal ${ }^{3}$ to the Fourier transform of $h(r)$, the so-called the liquid structure factor ${ }^{4}$

$$
S(q)=\frac{4 \pi \bar{\rho}}{q} \int_{0}^{\infty} h(r) r \sin (q r) \mathrm{d} r .
$$

The asymptotic behavior of $h(r)$ can be determined applying functional density theory [44, 62]. In this framework the total correlation function $h(r)$ can be deduced from the direct correlations based on the inter-particle potential (2.1). The total correlation function $h(r)$ and the direct correlation function ${ }^{5} c(r)$ are related via the OrnsteinZernike relation (OZR)

$$
h(r)=c(r)+\rho \int c\left(\left|\mathbf{r}-\mathbf{r}^{\prime}\right|\right) h\left(r^{\prime}\right) d \mathbf{r}^{\prime}
$$

\footnotetext{
${ }^{3}$ This is valid only in case of neutron scattering. In case of X-ray scattering the $q$-dependent atomic form factor has to be taken into account as discussed in chapter 6.

${ }^{4}$ This definition deviates from the mono-atomic structure factor (equation 6.19) usually given in textbooks [3] by not including self scattering of the atoms. This, however, is advantageous in case of X-ray scattering as will be discussed in chapter 6 .

${ }^{5}$ Equation (2.4) is often used to define $c(r)$.
} 

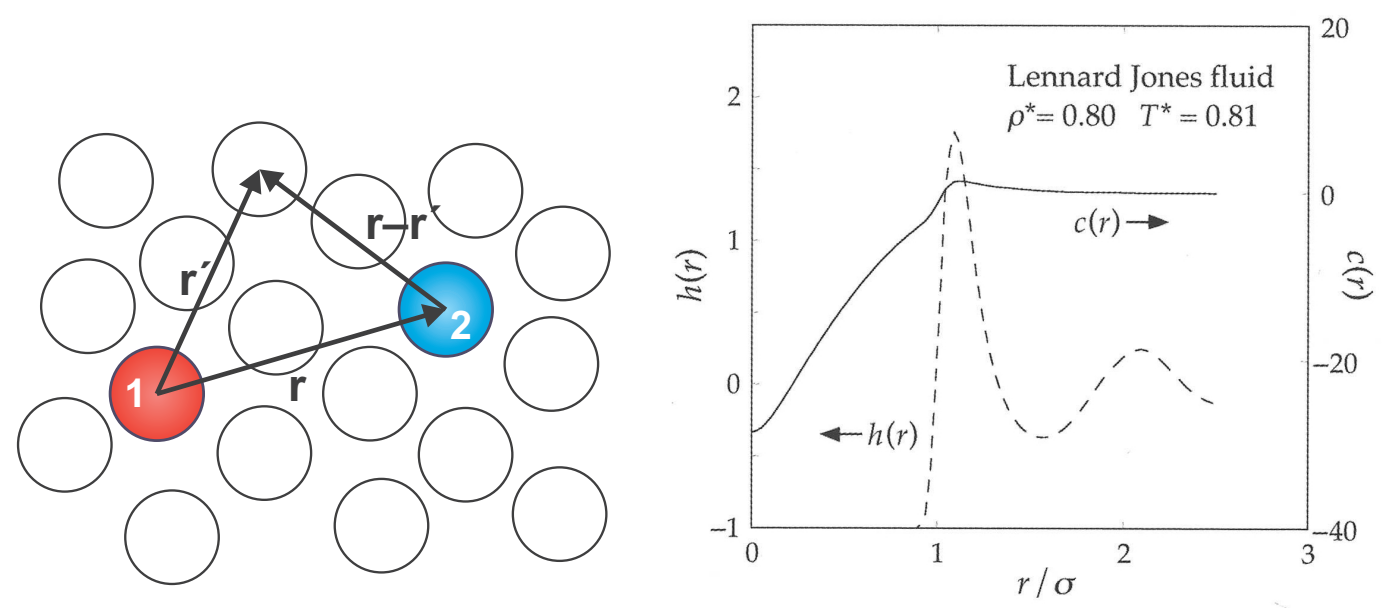

Figure 2.1: (a) The correlation between particle 1 and 2 is expressed by the OZR. It stems mainly from indirect correlations propagated by intermediate particles. (b) Total correlation function $h(r)$ and direct correlation function $c(r)$ obtained by Monte Carlo simulations for a Lennard-Jones fluid (taken from Hansen and McDonald [62]).

in a uniform and isotropic liquid [115]. This relation has the following physical interpretation (figure 2.1): the total correlation $h(r)$ between two particles is not only determined by the direct correlation $c(r)$ of the particles, but also by the indirect correlation propagated via a set of intermediate particles described by the second term in (2.4). The OZR can easily be generalized for multi-component systems [62] giving the partial total correlation functions

$$
h_{\alpha \beta}(r)=c_{\alpha \beta}(r)+\rho \sum_{\gamma} x_{\gamma} \int c_{\alpha \gamma}\left(\left|\mathbf{r}-\mathbf{r}^{\prime}\right|\right) h_{\gamma \beta}\left(r^{\prime}\right) d \mathbf{r}^{\prime}
$$

with the number concentration $x_{\gamma}=N_{\gamma} / N$ and the coefficients $\alpha, \beta$, and $\gamma$ representing the different constituents, e.g. cations and anions. The OZR can be solved in the mean spherical approximation where the direct correlation function is assumed to be proportional to the pair potential [62]

$$
c_{\alpha \beta}(r)=-\frac{1}{k_{\mathrm{B}} T} \phi_{\alpha \beta}(r) .
$$

Inserting (2.6) in (2.5) the OZR can be solved in Fourier space. In case of a binary mixture of cations and anions with a inter-particle potential of (2.1) neglecting the dispersion term, it can be shown $[44,91]$ that, asymptotically, all partial correlation functions yield either monotonic exponential decay

$$
r h_{\alpha \beta}(r) \sim A_{\alpha \beta} \exp \left(-\frac{r}{\xi}\right), \quad r \rightarrow \infty
$$

or an oscillatory behavior

$$
r h_{\alpha \beta}(r) \sim A_{\alpha \beta} \exp \left(-\frac{r}{\xi}\right) \cos \left(\frac{2 \pi r}{d}-\theta_{\alpha \beta}\right), \quad r \rightarrow \infty .
$$




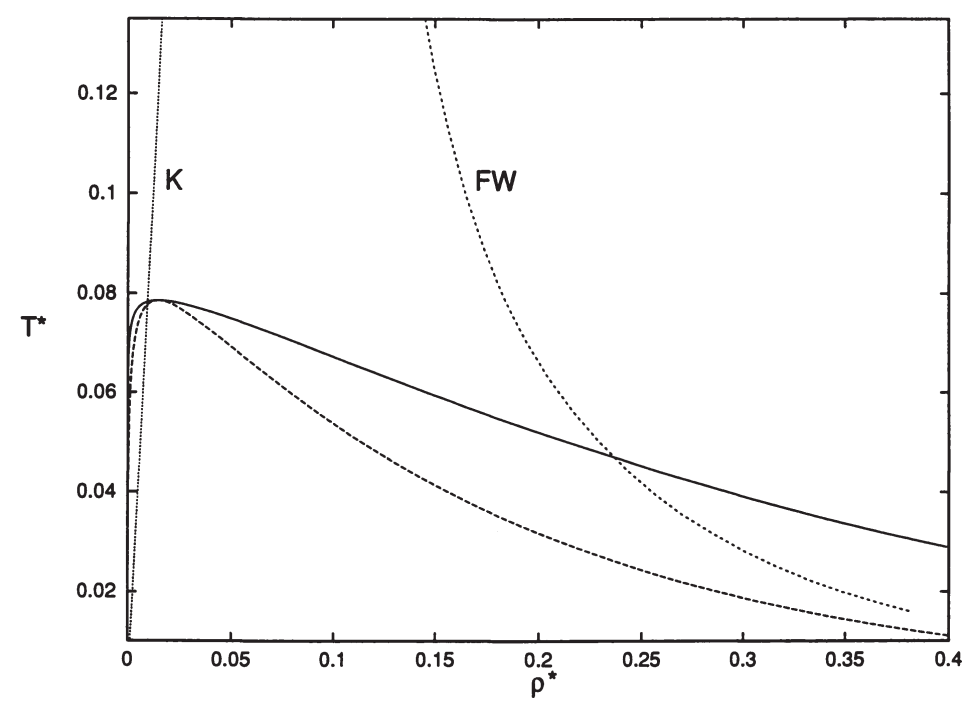

Figure 2.2: $\quad$ Phase diagram of a liquid consisting of charged hard spheres with equal diameter $R$ and charge $e Z$. The diagram is plotted vs. reduced temperature $T^{*}=k_{\mathrm{B}} \varepsilon R /(e Z)^{2}$ and total density $\rho^{*}=c R^{3}$. The solid line marks the liquid-vapor coexistence curve and the long-dashed curve is the related spinodals. The short dashed curve represents the Fisher Widom line (FW) and the dotted curve is the Kirkwood line (K). The cross-over lines were calculated using a generalized mean spherical approximation (taken from Evans and Leote de Carvalho [44]).

For all correlation functions the correlation length $\xi$ and the periodicity $d$ are the same, while the amplitude $A_{\alpha \beta}$ and phase $\theta_{\alpha \beta}$ are different. This also holds for the numbernumber total correlation function $h_{\mathrm{NN}}$ and the charge-charge total correlation function $h_{\mathrm{ZZ}}$ measuring the correlations in total number density and charge density, respectively [129]. Whether the asymptotic correlations are monotonous decaying (2.7) or oscillatory (2.8) depends on the thermodynamic state set by temperature and concentration. If Coulomb forces are present, charge oscillation occurs for small Debye screening length $\lambda_{\mathrm{D}}$ (cf. chapter 3), i.e. for $\lambda_{\mathrm{D}}$ being in the order of the particle diameter. The transition between monotonous and oscillatory decaying correlations is either called Fisher Widom line [50] or Kirkwood line [84]. The former is associated with transitions in number density, the other with transitions in concentration or charge density. Both transitions are represented by lines in the phase diagram (figure 2.2). In the presence of long-range dispersion forces, $h(r)$ will decay with $r^{-6}$ for $r \rightarrow \infty$. However, for high density, meaning high ion concentration in ILs, the oscillatory decaying term (2.8) dominates the correlation on intermediate range up to several intermolecular distances [44].

The partial total correlation functions (2.5) can be determined by scattering experiments (cf. chapter 6). The mesoscopic oscillatory correlations of form (2.8) lead to a so called first sharp diffraction peak at low wave vector transfer $q$. This phenomenon has been intensively studied in neutron scattering experiments of network-forming vitreous 
materials [129]. In ILs, however, a larger variety of correlations is present resulting in two or more low- $q$ peaks (cf. chapter4). These correlations originate from the large and asymmetric shape of the ions.

\subsection{Ion-ion correlations at the interface}

The interfacial structure of ILs at a solid wall results from the interplay between ion-wall interactions and ion-ion correlations. The ion-wall interactions include particle exclusion, van der Waals forces and Coulomb forces. Additionally, ions can be specifically adsorbed at the interface, e.g. due to hydrogen bonding and any physi- or chemisorption process. If the ion-wall interactions are short range or weak, the ion arrangement is dominated by ion-ion correlations already one or two molecular distances away from the wall. This is the case for Coulomb forces that are screened after the first ion layer, as the Debye length $\lambda_{\mathrm{D}}$ is usually smaller than an ionic diameter in ILs. Compared to Coulomb forces, van der Waals forces are weak. However, they are not directly screened and can influence the ions at long distance.

The general discussion of bulk correlations can be extended to interfacial correlations induced by short ranged ion-wall interactions. As stated in section 2.2, the total correlation function between a test particle and a second particle is dominated by interactions over intermediate particles. Thus, the potential of the test particle $\phi(r)$ can be considered as an external potential for the other particles. In analogy to the discussion in the previous section, a potential $V(z)$ of a wall acting on the particles at the interface should induce the same asymptotic behavior as in the bulk liquid. Indeed, one can show that the number density fluctuations $\rho(z)-\bar{\rho}$ from the mean number density $\bar{\rho}$ of a liquid adjacent to a wall has the asymptotic behavior [44]

$$
\rho(z)-\bar{\rho} \sim A_{\mathrm{W}} \exp \left(-\frac{z}{\xi}\right) \cos \left(\frac{2 \pi z}{d}-\theta_{\mathrm{W}}\right), \quad z \rightarrow \infty .
$$

The decay length $\xi$ and the periodicity $d$ are the same as in bulk while the amplitude $A_{\mathrm{W}}$ and phase $\theta_{\mathrm{W}}$ depend on the wall potential. The relation (2.9) has been calculated for a short range inter-particle potential without Coulomb forces and a wall potential decaying faster than the direct correlation function $c(r)$. However, the same asymptotic behavior should also hold for potentials including Coulomb forces as the the wall potential is screened on the sub molecular Debye length $\lambda_{\mathrm{D}}$ leading effectively to a short range interaction. So far, there has been only limited experimental evidence [102, 113]. 


\section{Chapter 3}

\section{The electric double layer}

At the liquid/solid interface between a charged wall and an electrolyte, the ions in the liquid form a so-called electric double layer (figure 3.1). The first layer consists of surface charge bound to the solid substrate. It can be comprised of electrons on metal electrodes and adsorbed ions in the so-called Stern layer. The second layer is formed by counter ions that are attracted from bulk liquid to the interface by Coulomb interaction. Thus, the electric field originating from the surface charge is screened and the bulk liquid is nearly field free. The electric double layer is of significant interest in surface and colloidal science $[22,97]$ and has been subject of investigation for a hundred years. In the early 20th century Gouy, Chapman and Stern developed a theory to describe the capacitance and the spatial ion distribution in diluted salt solutions near an electrode $[26,57,147]$. First attempts for extending this theory to highly concentrated solutions and molten salts were made in the early 50s, taking into account the finite size of ions and solvent molecules $[16,38,51,127]$. With the growing scientific interest in ionic liquids (ILs), i.e. electrolytes consisting solely of ions [167], this topic recently gained increasing attention [86]. In this chapter, the theory of Gouy Chapman and Stern is derived based of the Poisson-Boltzmann equation. As this theory is only valid for dilute salt solutions, some modified theories are presented thereafter, which are suitable for describing the electric double layer in highly concentrated electrolytes and ILs. Then, the dynamics of the double layer upon time dependent electrode potential variation is discussed. In fact, dilute and concentrated electrolytes show the same asymptotic response, illustrating its diffusive origin.

\subsection{Poisson-Boltzmann theory of the diffuse double layer}

Gouy and Chapman derived the electric potential in an electrolyte near a charged wall assuming a Boltzmann-distributed ion concentration. In this mean-field theory each ion is treated as a point charge interacting with the mean-field generated by the other ions. This model is only valid in the limiting case of dilute solutions and small potentials compared to the thermal excitation $k_{\mathrm{B}} T$. While it cannot describe the high ion concentrations of ILs, this model can be extended to highly concentrated electrolytes 

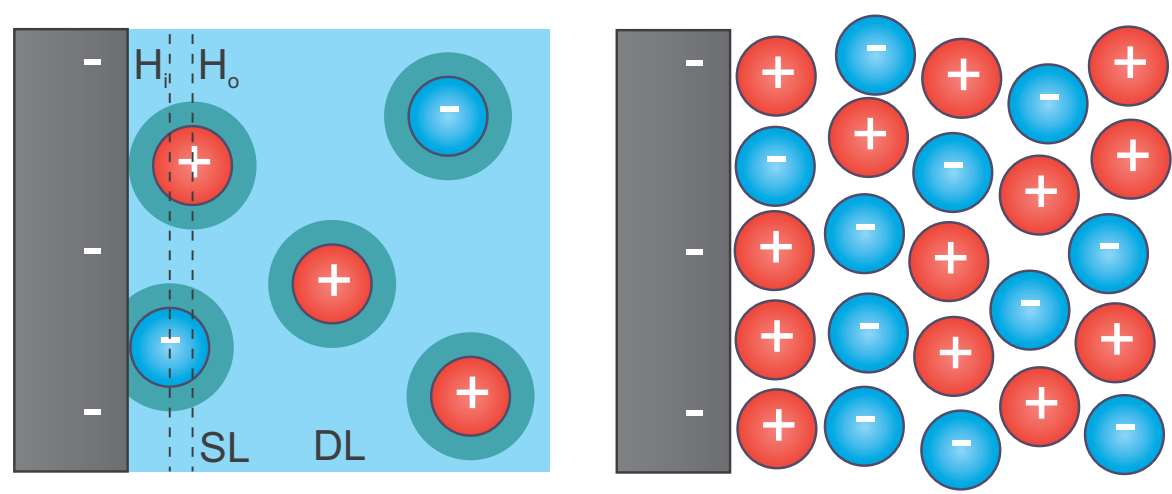

Figure 3.1: (a) Ion distribution in an aqueous solution near a charged wall. The Stern layer (SL) is located between the inner and the outer Helmholtz plane, $\mathrm{H}_{\mathrm{i}}$ and $\mathrm{H}_{\mathrm{o}}$ respectively. The diffuse layer DL is enriched with counter ions. (b) Ion distribution in an IL near a charged wall.

(cf. section 3.2).

The starting point to derive the structure of the diffuse double layer is electrostatics. In electrostatics the electric potential $\psi(z)$ is generally related to the charge $\rho_{\mathrm{c}}(z)$ via the Poisson equation ${ }^{1}$

$$
\frac{\mathrm{d}^{2}}{\mathrm{~d} z^{2}} \psi(z)=-\frac{\rho_{\mathrm{c}}(z)}{\varepsilon_{r} \varepsilon_{0}} .
$$

The charge can be written in terms of cation and anion concentrations $c_{\mathrm{c}}(z)$ and $c_{\mathrm{a}}(z)$, respectively, ${ }^{2}$

$$
\rho_{\mathrm{c}}(z)=e\left(c_{\mathrm{c}}(z)-c_{\mathrm{a}}(z)\right) .
$$

For dilute solutions the assumption of a Boltzmann distributed ion concentration is made

$$
c_{\mathrm{a} / \mathrm{c}}(z)=\bar{c} \mathrm{e}^{-\frac{W_{\mathrm{a} / \mathrm{c}}}{k_{\mathrm{B}} T}}
$$

where $\bar{c}$ is the mean concentration in the bulk solution. In a one dimensional problem the work $W_{\mathrm{a} / \mathrm{c}}$ is required to bring an anion/cation from bulk solution to a position near the charged wall. In the mean field of all other ions this work is $W_{\mathrm{a}}=-e \psi(z)$ for anions and $W_{\mathrm{c}}=e \psi(z)$ for cations. Combining equations (3.1)-(3.3) we obtain the Poisson-Boltzmann equation

$$
\begin{aligned}
\frac{\mathrm{d}^{2}}{\mathrm{~d} z^{2}} \psi(z) & =\frac{e \bar{c}}{\varepsilon_{r} \varepsilon_{0}}\left(\mathrm{e}^{\frac{e \psi(z)}{k_{\mathrm{B}} T}}-\mathrm{e}^{-\frac{e \psi(z)}{k_{\mathrm{B}} T}}\right) \\
& =\frac{2 e \bar{c}}{\varepsilon_{r} \varepsilon_{0}} \sinh \left(\frac{e \psi(z)}{k_{\mathrm{B}} T}\right)
\end{aligned}
$$

\footnotetext{
${ }^{1}$ As the potential at a planar wall is derived, only the one dimensional Poisson equation in $z$ direction normal to the wall is considered.

${ }^{2}$ assuming a monovalent salt
} 
For small potentials $\psi \ll k_{\mathrm{B}} T / e$ the exponential functions can be expanded leading to the linearized Poisson-Botzmann equation

$$
\frac{\mathrm{d}^{2}}{\mathrm{~d} z^{2}} \psi(z)=\frac{2 e^{2} \bar{c}}{\varepsilon_{r} \varepsilon_{0} k_{\mathrm{B}} T} \psi(z)
$$

Applied to the problem of a planar electrode, this differential equation can be solved easily with the boundary conditions of a fixed potential $\psi(0)=\psi_{0}$ at the electrode surface and a vanishing potential $\psi(z \rightarrow \infty)=0$ in bulk solution. The solution reads

$$
\psi(z)=\psi_{0} \mathrm{e}^{-z / \lambda_{D}},
$$

i.e. the electrode potential $\psi_{0}$ decays with the Debye screening length

$$
\lambda_{\mathrm{D}}=\sqrt{\frac{\varepsilon_{r} \varepsilon_{0} k_{B} T}{2 \bar{c} e^{2}}} .
$$

The Poisson-Boltzmann equation (3.4) can also be solved analytically. Its solution has the same asymptotic exponential decaying behavior, i.e. far away from the electrode, as the solution of the linearized problem (3.6). However, close to the electrode the potential decreases faster than the linearized solution [23]. This effect is more pronounced for high electrode potentials.

Neither the potential $\psi(z)$ nor the concentration profile can be measured directly in a simple way. However, an experimentally accessible quantity of the double layer is its differential capacitance

$$
C\left(\psi_{0}\right)=\frac{\mathrm{d} \sigma\left(\psi_{0}\right)}{\mathrm{d} \psi_{0}}
$$

$C\left(\psi_{0}\right)$ gives the change of the total charge

$$
\sigma\left(\psi_{0}\right)=-\int_{0}^{\infty} \rho_{c}\left(z, \psi_{0}\right) \mathrm{d} z
$$

in the double layer and on the electrode, respectively, by variation of the electrode potential $\mathrm{d} \psi_{0}$. The differential capacitance can be calculated from the total charge in the double layer. In case of the Poisson-Boltzmann equation (3.4) the total charge is given by the Grahame equation

$$
\sigma\left(\psi_{0}\right)=\sqrt{8 \bar{c} \varepsilon_{r} \varepsilon_{0} k_{\mathrm{B}} T} \sinh \left(\frac{e \psi_{0}}{2 k_{\mathrm{B}} T}\right) .
$$

Inserting (3.10) in (3.8) gives the differential capacitance according to the PoissonBoltzmann equation

$$
C_{\mathrm{PB}}=\frac{\varepsilon_{r} \varepsilon_{0}}{\lambda_{\mathrm{D}}} \cosh \left(\frac{e \psi_{0}}{2 k_{\mathrm{B}} T}\right)
$$

Equation (3.11) has a parabolic potential dependence with a minimum at the potential of zero charge (PZC), i.e where $\sigma=0$. 


\section{Stern layer}

In order to explain discrepancies between theory and experimental data, there have been a lot of corrections and amendments to the Gouy-Chapman model, that corrects for the unphysically divergence of $C_{\mathrm{PB}}$ at high potentials. The most prominent extension is the Stern layer. Stern combined the models of Gouy, Chapman and Helmholtz by introducing an adsorbed layer, the so-called Stern layer with a potential independent capacitance $C_{\mathrm{S}}$, in addition to the diffuse layer. Thus, the total differential capacitance is given by a combination of two capacitors in series

$$
\frac{1}{C}=\frac{1}{C_{\mathrm{S}}}+\frac{1}{C_{\mathrm{PB}}} .
$$

The constant $C_{\mathrm{S}}$ counteracts the divergent $C_{\mathrm{PB}}$ at high potentials and, thus, limits the total differential capacitance. The capacitance of a layer of adsorbed ions can be estimated by

$$
C_{\mathrm{S}}=\frac{\varepsilon_{r} \varepsilon_{0}}{d_{0}-z_{\sigma}}
$$

where $d_{0}$ is the center of charge of the adsorbed layer in respect to the interface and $z_{\sigma}$ the position of the surface charge on the electrode. The effective dielectric constant $\varepsilon_{r}$ equals not necessarily the bulk dielectric constant. Grahame refined this model for aqueous solutions by distinguishing between an inner Helmholtz layer of specifically adsorbed ions and an outer Helmholtz layer of solvated ions [58]. In lack of solvent molecules in ILs the Stern layer only consists of surface adsorbed ions.

\subsection{Diffuse double layers in ionic liquids}

For ILs the assumptions made by Gouy and Chapman are certainly not valid anymore. The adopted Boltzmann distribution of ions leads to unphysically high ion concentrations near the IL/electrode interface. Due to the finite size of the ions, steric effects will govern the concentration profile at elevated electrode potential, i.e. the ion concentration cannot exceed a limiting value at the interface (figure 3.3). This problem is well-known in the theory of the electric double layer in concentrated electrolytes and molten salts. In this scope steric effects were first considered by Bikerman [16] and independently by Eigen and Wicke [37, 38]. Subsequently, Freise solved a modified Poisson-Boltzmann equation of a diffuse double layer and derived a differential capacitance differing substantially from the capacitance of the Gouy-Chapman model. Freise's model predicts a maximum at the PZC in contrast to the minimum predicted by the Gouy-Chapman model. With the emerging interest in highly concentrated electrolytes and ILs the model of Bikerman and Freise received attention anew in an article by Kornyshev [86]. Kornyshev adopted the so called lattice gas model, previously developed by Borukhov [18], to derive the ion distribution in ILs near a charged wall previously found by Bikerman

$$
c_{\mathrm{a} / \mathrm{c}}(z)=\bar{c} \frac{\mathrm{e}^{ \pm \frac{e \psi_{0}}{2 k_{\mathrm{B}} T}}}{1-\gamma+\gamma \cosh \left(\frac{e \psi_{0}}{2 k_{\mathrm{B}} T}\right)} .
$$




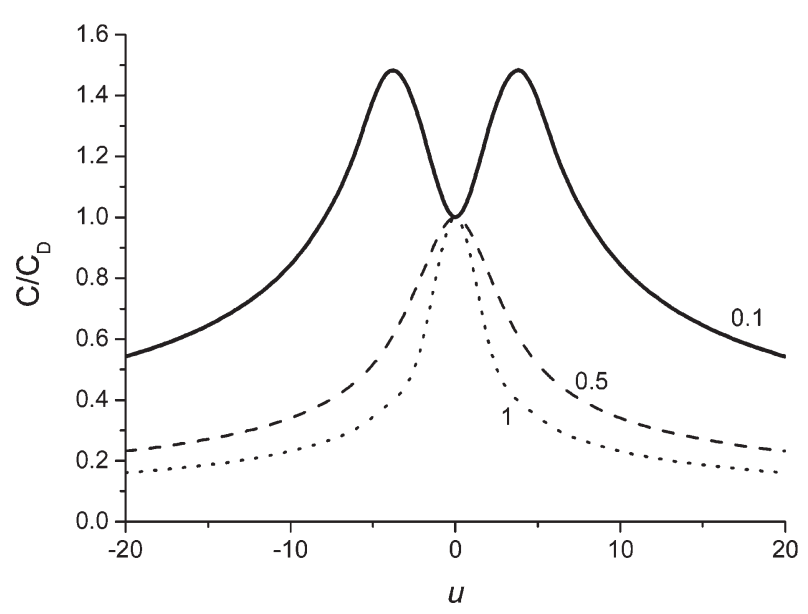

Figure 3.2: Differential capacitance of the modified Poisson-Boltzmann equation (3.17) for different parameters $\gamma$. For $\gamma \rightarrow 0$ the capacitance (3.11) according to the PoissonBoltzmann equation is retrieved (taken from Kornyshev et al. [86]).

Here the parameter $\gamma$ is a measure of the maximal ion excess at the interface

$$
\gamma=\frac{2 \bar{c}}{c_{\max }}
$$

given by the ratio of the mean ion concentration in bulk $\bar{c}$ and the maximal ion concentration at the interface $c_{\max }$. The ion distribution (3.14) resembles a Fermi distribution, thus limiting the concentration for large potentials to $c_{\max }$ (dashed line in figure 3.4a). In the limit of infinite maximal interfacial ion concentration $c_{\max }$, i.e. $\gamma \rightarrow 0$, equation (3.14) approaches the Boltzmann distribution (3.3).

Combining equations (3.1), (3.2), and (3.14) leads to a modified Poisson-Boltzmann equation

$$
\frac{\mathrm{d}^{2}}{\mathrm{~d} z^{2}} \psi(z)=\frac{2 e \bar{c}}{\varepsilon_{r} \varepsilon_{0}} \frac{\sinh \left(\frac{e \psi(z)}{k_{\mathrm{B}} T}\right)}{1+2 \gamma \sinh ^{2}\left(\frac{e \psi(z)}{2 k_{\mathrm{B}} T}\right)} .
$$

which can be solved analytically [86]. The differential capacitance in this model reads

$$
C_{\mathrm{MPB}}=\frac{C_{\mathrm{PB}}}{1+2 \gamma \sinh ^{2}\left(\frac{e \psi_{0}}{2 k_{\mathrm{B}} T}\right)} \sqrt{\frac{2 \gamma \sinh ^{2}\left(\frac{e \psi_{0}}{2 k_{\mathrm{B}} T}\right)}{\ln \left[1+2 \gamma \sinh ^{2}\left(\frac{e \psi_{0}}{2 k_{\mathrm{B}} T}\right)\right]}} .
$$

The shape of $C_{\mathrm{MPB}}$ strongly depends on the concentration limiting parameter $\gamma$ (figure 3.2). For $\gamma=0$ the differential capacitance $C_{\mathrm{PB}}$ of the Gouy-Chapman model is retrieved, i.e. an infinite amount of ions can be dragged to the interface. For any finite value of $\gamma$ the capacitance decreases for high potentials $\psi_{0}$ as

$$
C_{\mathrm{MPB}} \approx \frac{\varepsilon_{r} \varepsilon_{0}}{\lambda_{\mathrm{D}}} \sqrt{\frac{k_{\mathrm{B}} T}{2 \gamma e \psi_{0}}}
$$

This $x^{-1 / 2}$ dependence is typical for lattice saturation, i.e. for high potentials all interfacial sites are occupied by counter ions and the double layer has to expand additionally. 

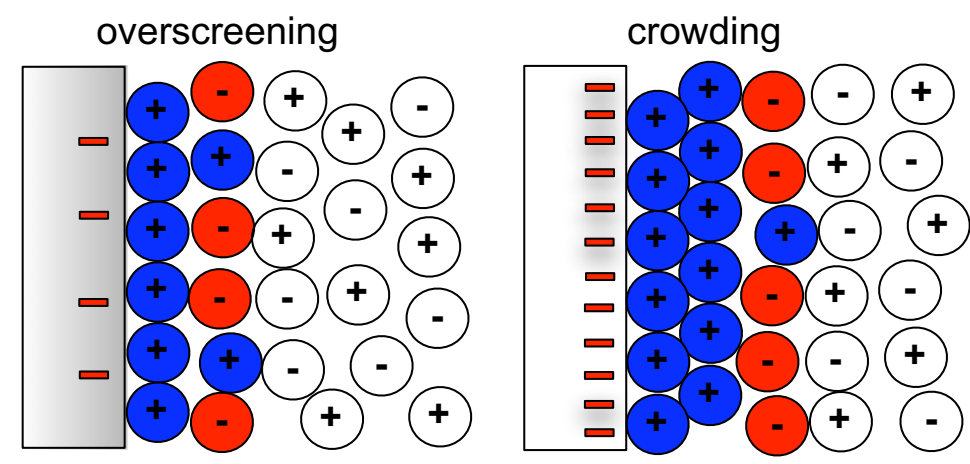

Figure 3.3: Ion distribution in an IL near a charged wall. (a) For low potentials the charge at the electrode is over-screened by counter ions. (b) For high potentials the excess of counter ions leads to crowding of ions (taken from Bazant et al. [13]).

In literature this is referred to as crowding (figure 3.3b). A curiosity of this model is the transition from a camel back shaped capacitance curve to a bell shaped curve with a maximum at the PZC at $\gamma=1 / 3$. This transition was reproduced by MD simulations [46] in which the IL was modeled by spherical charged beats and, in a separate simulation, by chains consisting of charged and uncharged beats. For the first model a bell shaped differential capacitance was found, while the later exhibited a camel back shaped curve. The authors concluded that the uncharged beats act as voids in the lattice gas model and thus enhancing the maximal ion concentration at the interface in respect to the bulk.

In the previously discussed models the intrinsic structure of liquids (cf. chapter 2) was totally neglected. Ions are assumed to be point-like charges interacting via a meanfield. Also the introduction of a maximal concentration at the interface is a phenomenological concept which does not include ion-ion correlations. In order to incorporate ion-ion correlations, again in a phenomenological way, Bazant extended the model of Bikerman and Freise based on a Landau-Ginzburg like continuum theory [13]. He introduced a correlation length $l_{\mathrm{c}}$ describing ion-ion correlations on a molecular scale. The modified Poisson-Boltzmann equation becomes a fourth order differential equation

$$
\left(1-l_{\mathrm{c}}^{2} \frac{\mathrm{d}^{2}}{\mathrm{~d} z^{2}}\right) \frac{\mathrm{d}^{2}}{\mathrm{~d} z^{2}} \psi(z)=\frac{2 e \bar{c}}{\varepsilon_{r} \varepsilon_{0}} \frac{\sinh \left(\frac{e \psi(z)}{k_{\mathrm{B}} T}\right)}{1+2 \gamma \sinh ^{2}\left(\frac{e \psi(z)}{2 k_{\mathrm{B}} T}\right)}
$$

which cannot be solved analytically anymore. Numerically derived ion densities and concentration distributions are shown in figure 3.4. The fourth order differential equation leads to damped charge oscillations which represent the ion-ion correlations described in chapter 2. In this context the oscillations are referred to as overscreening (figures 3.3a). This term describes the fact that the excess ions next to the charged wall exceeds the surface charge on the electrode. The differential capacitance has a similar potential dependence compared to the model of Bikerman and Freise (figure 4.4b). For small potentials, however, the differential capacitance is significantly reduced. 

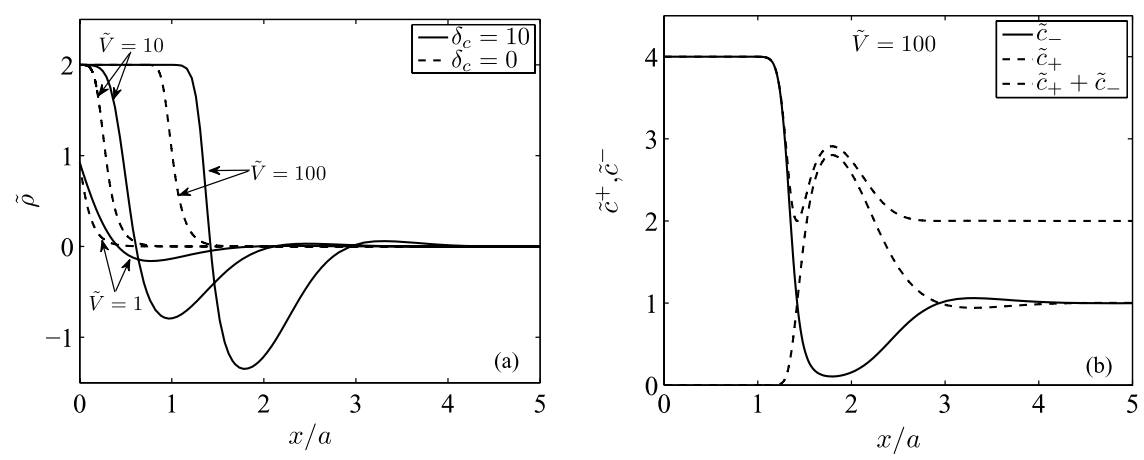

Figure 3.4: (a) The dimensionless charge densities $\tilde{\rho}$ of the electric double layer at different potentials $\tilde{V}=e V / k_{\mathrm{B}} T$ are compared for two dimensionless correlation lengths $\delta_{\mathrm{c}}=l_{\mathrm{c}} / \lambda_{\mathrm{D}}$. The charge density was derived after equation (3.19) from Bazant et al. [13]. $\delta_{\mathrm{c}}=0$ corresponds to the lattice gas model (3.14). (b) Cation concentration $\tilde{c}_{+}$and anion concentration $\tilde{c}_{-}$for a dimensionless correlation length of $\delta_{\mathrm{c}}=10$ at potential $\tilde{V}=100$ (taken from Bzant et al. [13]).

\subsection{Charging/discharging dynamics}

The dynamics of the double layer at the electrode can be investigated by electrochemical methods such as impedance spectroscopy, chrono amperometry or cyclic voltammetry (cf. chapter 7). In these methods, the response to a time-dependent voltage is usually analyzed by fitting the data to an equivalent circuit which consist of capacitors, resistors and other elements. The response is mainly governed by diffusion. Thus, the timescales are much longer than molecular relaxations or spontaneous collective excitations on timescales of the plasma frequency

$$
\omega_{P}=\sqrt{\frac{4 \pi n Z_{i}^{2} e^{2}}{\varepsilon_{0} \varepsilon m_{i}}} .
$$

This equation includes the number density $n$, the ion charge number $Z_{i}$, the elementary charge $e$, vacuum permittivity $\varepsilon_{0}$, the relative permittivity $\varepsilon$ and the ion mass $m_{i}$. For instance, with an estimate of $\varepsilon \approx 10$ the plasma frequency of the IL [tba $]^{+}[\mathrm{FAP}]^{-}$is about $0.5 \mathrm{THz}$.

Diffusion phenomenons can be treated in terms of thermodynamics. Any spatial gradient in the chemical potential leads to a diffusive flow of ions. Assuming the phenomenological hypothesis of linear response, the flux $J_{\alpha}$ of an ion species $\alpha$ is proportional to the gradient of the chemical potential $\mu_{\alpha}$. This leads to Fick's first law ${ }^{3}$ of diffusion

$$
J_{\alpha}=-\frac{D_{\alpha}}{k_{\mathrm{B}} T} c_{\alpha} \nabla \mu_{\alpha}
$$

\footnotetext{
${ }^{3} \mathrm{~A}$ more common form for dilute solutions without electric fields can be obtained by applying $\mu_{\alpha}=$ $k_{\mathrm{B}} T \ln c$
} 
where $D_{\alpha}$ is the diffusion coefficient ${ }^{4}$ and $c_{\alpha}$ the concentration. The time evolution of the concentration is given by Fick's second law

$$
\frac{\partial c_{\alpha}}{\partial t}=-\nabla J_{\alpha}=\nabla\left(\frac{D_{\alpha}}{k_{\mathrm{B}} T} c_{\alpha} \nabla \mu_{\alpha}\right) .
$$

Equation (3.22) represents the differential form of mass conservation in absence of any chemical reaction. To establish a set of differential equations describing the diffusion process, one needs to determine the chemical potential of an electrolyte system in dependence of ion concentration and electrode potential. The chemical potential of a thermodynamic system is given by the derivative of the Helmholtz free energy $F$ in respect to the number of particles $N$. This relation can also be written in terms of concentration $c$ and the Helmholtz free energy $f$ per volume

$$
\mu=\left.\frac{\partial F}{\partial N}\right|_{T, V}=\left.\frac{\partial f}{\partial c}\right|_{T, V} .
$$

In an electrolyte the chemical potential of an ion species $\alpha$ yields [14]

$$
\mu_{\alpha}=k_{\mathrm{B}} T \ln c_{\alpha}+Z_{\alpha} e \psi+\mu_{\alpha}^{\mathrm{ex}} .
$$

The first term is the chemical potential of a dilute, or ideal solution while the second term gives the influence of the local electric potential. The excess chemical potential $\mu_{\alpha}^{\mathrm{ex}}$ describes the deviation from the ideal value which can include steric effects and ion-ion correlations. ${ }^{5}$

For diluted solutions the ion-ion interactions are negligible and the excess chemical potential turns to zero. Thus the chemical potential given by (3.24) yields

$$
\mu_{\alpha}^{\text {dil }}=k_{\mathrm{B}} T \ln c_{\alpha}+Z_{\alpha} e \psi
$$

Inserting the chemical potential (3.25) into (3.22) gives the Nernst-Planck equation

$$
\frac{\partial c_{\alpha}}{\partial t}=D_{\alpha}\left[\nabla^{2} c_{\alpha}+\frac{Z_{\alpha} e}{k_{\mathrm{B}} T} \nabla\left(c_{\alpha} \nabla \psi\right)\right] .
$$

Together with the Poisson-Boltzmann equation (3.4), it describes the time evolution of the concentration profile in a dilute solution. These so called Poisson-Nernst-Planck equations form a set of differential equations. Applying appropriate boundary conditions these equations can be solved for model systems. For instance, Bazant et al. performed a comprehensive analysis of the response of such a model system to timedependent voltages [15]. It is comprised of a dilute electrolyte in between two parallel plate blocking electrodes. The evolution in time of charge density and potential is

\footnotetext{
${ }^{4}$ The diffusion coefficient $D_{\alpha}$ is connected to the mobility $u_{\alpha}$ via the Einstein relation $D_{\alpha}=k_{\mathrm{B}} T u_{\alpha}$

${ }^{5}$ The chemical potential can also be written in terms of activities: $\mu=k_{\mathrm{B}} T \ln a+Z e \psi$ where the activity $a=\lambda c$ includes the ion-ion interactions via the chemical activity coefficient $\lambda$ which is connected to the excess chemical potential $\mu^{\mathrm{ex}}=k_{\mathrm{B}} T \ln \lambda$.
} 

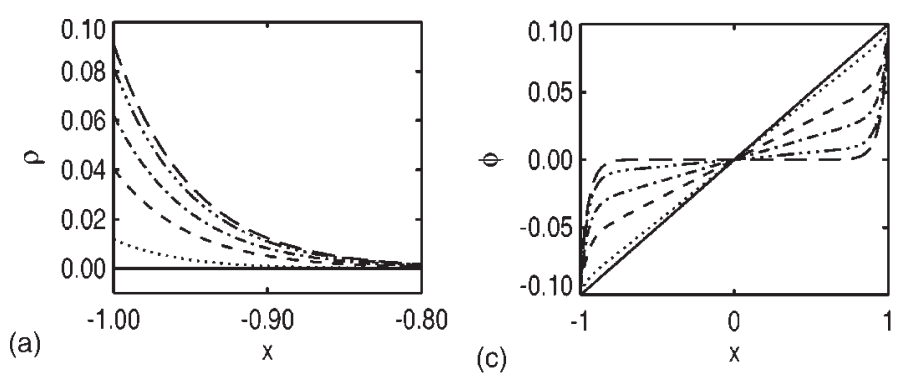

Figure 3.5: Charge profiles (a) and potential $\phi$ (c) between two electrodes after a potential step for times $t / \tau_{\mathrm{c}}=0$ (solid), 0.1 (dot), 0.5 (dash), 1 (dot-dash), 2 (dot-dotdot-dash) and $\infty$ (long dash) (taken from Bazant eta. [15]).

shown in figure 3.5. Besides showing numerical results, Bazant et al. also investigated the response to small excitations in the "weakly nonlinear" limit by matched asymptotic expansions. At leading order, the model system behaves like a $R C$ circuit with a response time

$$
\tau_{\mathrm{c}}=\frac{\lambda_{\mathrm{D}} L}{2 D} .
$$

Equation (3.27) reveals that the response depends on the microscopic Debye length $\lambda_{\mathrm{D}}$ and the macroscopic separation $L$ of the electrodes. Although this result is known for a long time [101] it is not present in some standard textbooks [97], where only the Debye time

$$
\tau_{\mathrm{D}}=\frac{\lambda_{\mathrm{D}}^{2}}{D}
$$

is stated. Compared to $\tau_{\mathrm{c}}, \tau_{\mathrm{D}}$ is rather small (ns to $\mu$ s for aqueous solutions) and gives the response of the diffuse layer itself to a given potential.

The analysis of the dynamic behavior of electrolyte electrode systems is not only limited to dilute solutions. Ion-ion interactions can be included via the excess chemical potential $\mu_{\alpha}^{\text {ex }}$ in equation (3.24). Following this approach Kilic et al. derived modified Poisson-Nernst-Planck equations from (3.16) for the model of Bikerman and Freise [82, 83]. Again, the leading order response time is given by $\tau_{\mathrm{c}}$ of equation (3.27). A comprehensive overview of induced charge kinetics in concentrated solutions can be found in [14]. Recently, Zhao solved the modified Poisson-Nernst-Planck equations resulting from Bazant's modified Poisson-Boltzmann equation (3.19) [177]. In this case, the included correlations cause a reduction of the leading order response time

$$
\tau_{c}^{*}=\frac{\lambda_{\mathrm{D}}^{3 / 2} L}{2 D l_{\mathrm{c}}^{1 / 2}},
$$

which depends on the correlation length $l_{\mathrm{c}}$. 


\section{Chapter 4}

\section{Structure and dynamics of ionic liquids}

In this chapter the recent findings of experimental and simulation work on structure and dynamics of ILs are presented. First, an overview of IL bulk properties is given. A common observation is the presence of inter-molecular correlations on several length-scales. These correlations also influence their transport properties, which are discussed in the following section. Here, the contributions of electrochemical experiments to the understanding of structure and ion transport properties are presented. Finally, the studies on IL/solid interfaces are discussed. Those studies reveal that the interfacial structure is governed by strong correlations as well. Thereafter, the impact of these correlations on the electrochemical quantities of IL/electrode systems is examined. For more detailed information the reader is referred to the recent review of ILs at charged interfaces published by Fedorov and Kornyshev [48].

\subsection{Bulk properties}

\section{Microscopic structure}

$\mathrm{X}$-ray and neutron scattering are classical techniques for studying the bulk structure of vitreous matter [49]. Especially neutron scattering is an established technique to resolve the structure of liquids. With the help of isotope variation, it is possible to determine the partial structure factors of polyatomic liquids [127]. However, the complex molecular structure of ILs hampers a quantitative separation of intra- and inter-molecular structure just by means of partial deuteration of ions. This task can only be accomplished with the help of advanced data analysis based on MD simulations. This approach is applicable to the data analysis of both, neutron and X-ray scattering data.

Systematic diffraction and simulation studies on the influence of the cation alkyl chain length on the bulk structure have been made for various ILs, such as imidazolium $\mathrm{PF}_{6}$-based ILs [5, 63], pyrrolidinium TFSI-based ILs [78, 80, 93], protic alkylammonium nitrate-based ILs [146], and ammonium TFSI-based ILs [144]. A common observation is the presence of two to three inter-molecular correlation lengths governing the radial distribution function $g(r)$. These correlations result in peaks in the structure fac- 

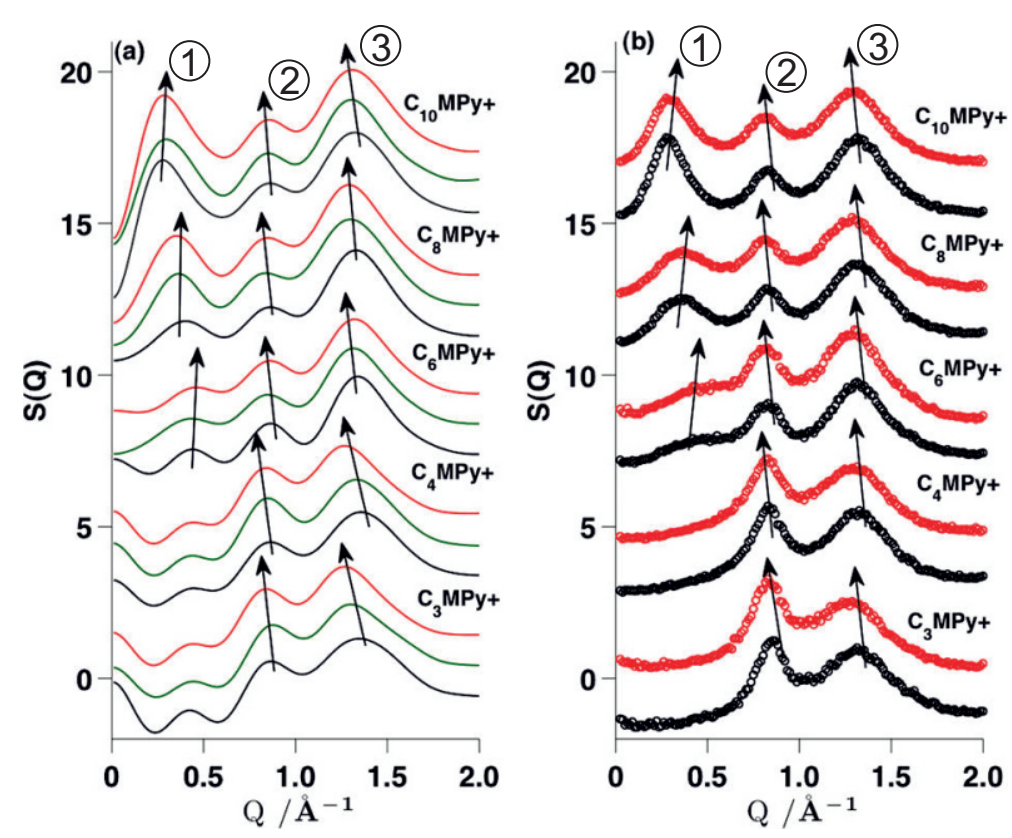

Figure 4.1: MD simulation (a) and small angle X-ray scattering (SAXS) data (b) for pyrrolidinium TFSI based ILs with different chain lengths. Arrows indicate the temperature dependence of the peaks while raising the temperature from $298 \mathrm{~K}$ to $363 \mathrm{~K}$ (taken from Li et al. [93]).

tor $S(q)$ at low $q$-values (figure 4.1). Oscillations at higher $q$-values, typically $>2 \AA^{-1}$, originate mainly from their intra-molecular structure $[79,132]$. The highest- $q$ peak $(3$ in figure 4.1) of the inter-molecular structure peaks mainly arises from the nearest neighbor correlation of oppositely charged ions. The second peak $\mathbf{2}$ is assigned to the real space correlation and anticorrelations of like ions, which can spread over several molecular distances [78]. The lowest- $q$ peak 1 arises from correlations of polar and apolar regions comprised of aggregated alkyl chains and ionic head groups, respectively [5, 128, 160]. Therefore, its intensity and position strongly depends on the alkyl chain length of the cation. For side chains smaller than a propyl-group this peak completely vanishes.

The temperature dependence of the peaks shown in figure 4.1 is noteworthy. The two high- $q$ peaks shift to lower $q$-values with increasing temperature corresponding to thermal expansion of the IL. However, the low- $q$ peak shifts inversely to higher $q$-values indicating thermal contraction. This negative thermal expansion has also been observed at the free surface with resonant soft X-ray reflectivity [106]. This phenomenon has been assigned to the increased disordered conformation of the alkyl chains with increasing temperature leading to a contraction of the apolar regions. The mean distance between the molecules, however, increases so that the overall volume expansion is positive. 


\section{Bulk ion dynamics}

The existence of long-range bulk correlations is supported by the findings applying spectroscopic techniques. For instance, utilizing coherent anti-Stokes Raman scattering (CARS), a series of imidazolium $\mathrm{PF}_{6}$-based ILs showed microscopic optical inhomogenieties suggesting mesoscopic local structures of several tens of nanometers [73, 140]. Also, collective motions of locally ordered domains in imidazolium and ammoniumbased ILs were observed with different techniques, such as heterodyne-detected Ramaninduced Kerr effect spectroscopy (OHDE-RIKES) [69, 128, 173], low-frequency Raman spectroscopy [73, 125], THz spectroscopy [174], optical Kerr effect measurements and dielectric relaxation spectroscopy $[68,163]$.

Further, combined time-dependent fluorescence Stokes shift experiments and MD simulations revealed complex solvation dynamics around the dye coumarin 153 in a imidazolium-based IL [152]. The origin of the solvation dynamics response was identified with the translation dynamics of the $\mathrm{BF}_{4}$ anion in and out of the dye solvation shell. This translational origin is in contrast to the mechanism in conventional polar liquids in which solvent rotations dominate the response and indicates the existence of microscopic structures.

\section{Charge transport and diffusion}

In a series of publications, Tokuda et al. systematically investigated diffusion-based properties of various ILs. This was done under variation of several parameters: anionic species in imidazolium-based ILs [155], alkyl chain length in imidazolium TFSI-based ILs [156], and the cation structure in TFSI-based ILs [157]. A summary of the findings is given in [158]. In these studies all diffusion-related quantities such as viscosity, selfdiffusion coefficients and conductivity showed a Vogel-Fulcher-Tammann temperature dependence [148] whereas the density decreased linearly with temperature. The cations' diffusion coefficients were found to be higher compared to the respective anions, in spite of the cations' larger size. This finding was confirmed by MD simulations [124]. Further, Tokuda et al. compared the conductivity values with calculated values from self diffusion coefficients measured with pulsed-field-gradient spin-echo NMR. This was done by using the Nernst-Einstein relation [62]. From the discrepancy between the values determined by conductivity measurements and NMR, the authors concluded that only a part of the ions is dissociated in ILs and contributes to the conductivity. However, the diffusion coefficient was found to be coupled to the ion conductivity not in a simple way [169]. Thus, the conclusion of Tokuda et al. has to be considered with great care.

The diffusion of various tracer solutes in ILs was studied by Kaintz et al. [77]. They compared measured friction coefficients from pulsed-field gradient NMR with predicted values from the Stokes-Einstein relation. For tracer molecules of the same size and larger than the IL solvent, the experimental data is in good agreement with theory. However, for smaller molecules deviations were observed. Small neutral tracer molecules were found to be more mobile than predicted, whereas small charged tracer molecules, especially lithium ions, are much less mobile. This tracer diffusion behav- 
ior is quite similar to what has been observed in conventional solvents. However, the mobility did not evidently depend on the degree of micro-phase separation in polar and apolar regions. Thus, the authors concluded that the difference of the diffusion coefficients originates from subtle packing effects and not from inter-molecular interactions. Applying dye rhodamine $6 \mathrm{G}$ as tracer solute, a fast and a slow diffusion process were observed in pyrrolidinium TFSI-based ILs [60]. The respective fluorescence correlation spectroscopy study comprised ILs of various alkyl chain lengths. The two diffusion coefficients deviate about a factor of ten. The existence of two processes was ascribed to the presence of polar and apolar micro-phases.

Similar findings were made by applying elastic and inelastic neutron scattering. In a study by Triolo et al. also two processes were identified in 1-butyl-3-methylimidazolium $\mathrm{PF}_{6}$ [161]. The first is an Arrhenius activated Debye-like process, observed at low temperatures, which may be connected to methyl and butyl chain relaxation. Another high temperature diffusive process exhibit a Vogel-Fulcher-Tammann temperature dependence.

\subsection{Electrochemistry experiments}

Electrochemical experiments provide valuable information of the electrolytic properties of ILs such as conductivity [155], differential capacitance [95, 96, 149], relaxation times [32] and diffusion coefficients [85]. Such measurements are often performed supplementary. In publications raw data only is provided in the supporting information, if at all. However, the way of extracting physical quantities from such electrochemical experiments is controversially discussed in literature. For instance, very different differential capacitance curves of similar ILs at the same electrode were reported by different authors (figure 4.2a). These discrepancies might originate from subtle differences of electrode surface qualities, which varies even for same electrode materials. MD simulations have shown that e.g. electrode roughness has an immense impact on the differential capacitance of ILs at graphite and gold electrodes [65]. At a rough surface, differential capacitance curves were found to exhibit more features than on an atomically flat surface. Further, the electronic properties of the electrode material have a huge impact on the differential capacitance. In an impedance spectroscopy study of 1butyl-3-methylimidazolium TFSI, the influence of electrode materials was investigated [24]. The electrode materials included platinum, glassy carbon, boron doped diamond, and carbon nitride. Another study on the influence of semiconducting electrodes is presented in [72]. Unfortunately, no raw impedance data was provided in these studies. Another explanation for the discrepancy of reported data might be of systematic quality $[32,65]$. For instance, probing the electronic response with only a single frequency leads to ambiguous results as illustrated in figure $4.2 \mathrm{~b}$ where the temperature dependence of broadband impedance spectra of an IL is shown plus the quasi temperature dependence of a single frequency measurement [71, 111].

The determination of diffusion coefficients from impedance measurements is con- 

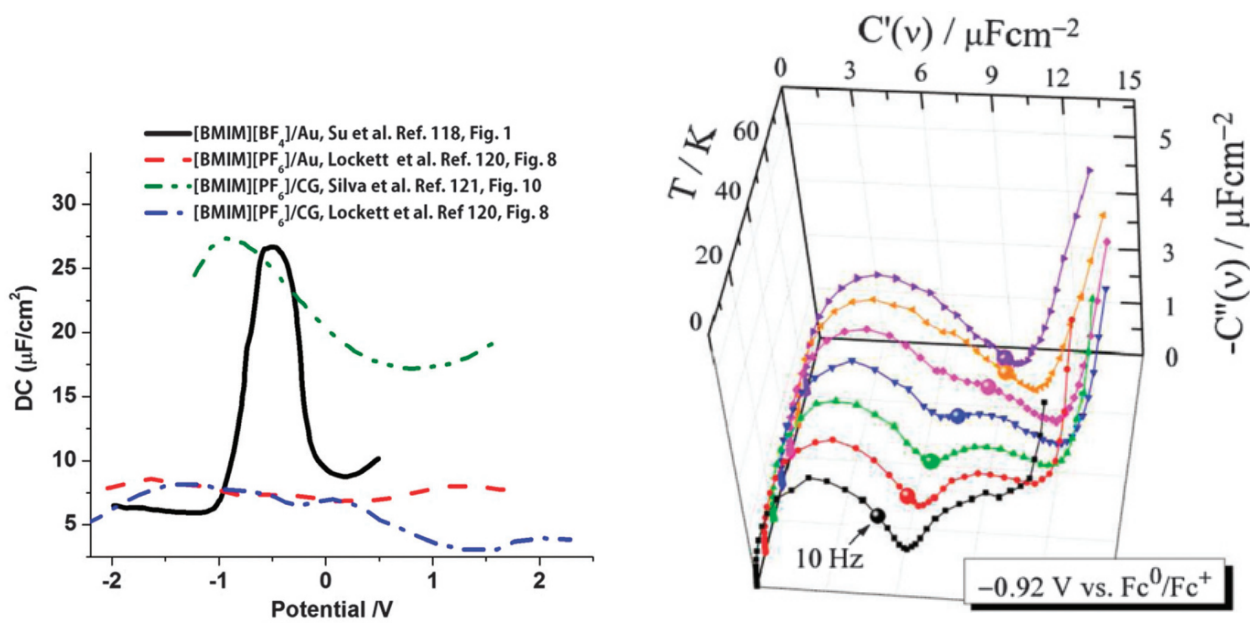

Figure 4.2: (a) Comparison of differential capacitance from different single frequency experiments (taken from $\mathrm{Hu}$ et al. [65]). (b) Broad band impedance spectroscopy data at different temperatures. Marked dot indicates the complex capacitance of the frequency $10 \mathrm{~Hz}$ (taken from Drüschler et al. [32]).

troversial as well. Comparison of impedance results with pulsed-field gradient NMR showed discrepancies of over four orders of magnitude [169]. These findings are in contrast to another study where results match well with pulsed-field gradient NMR measurements $[130,131]$. Here the charge transport was assigned to a much faster process and interpreted in terms of charge carrier hopping in an energy landscape [35]. However, this process was only observed at low temperatures where the IL can be assumed to be in a glass-like state and electrode polarization is strongly reduced.

\section{Impedance spectroscopy}

Broadband impedance spectroscopy was used by several groups to study the electric response of various IL/electrode systems. Applying a three electrode setup, the effect of a constant bias potential was examined. For instance, the Roling group observed three capacitive processes at the interface of the IL $[\mathrm{bmpy}]^{+}[\mathrm{FAP}]^{-}$and a single-crystalline gold, (111) face, electrode [8]. A fast process on millisecond timescale was ascribed to double layer formation while a ultra-slow process was associated with specific ion adsorption. The intermediate process was found to be heavily potential dependent and was tentatively ascribed, with the help of STM measurements, to Herringbone reconstruction of Au surface. In a subsequent publication the temperature dependence of the processes was studied [32]. A Vogel-Fulcher-Tammann temperature dependence was found for the fast process as well as for the ion conductivity, whereas the intermediate process shows a Arrhenius temperature dependence. With these findings, the authors concluded that the fast process is diffusion driven while the other process is connected to interface relaxations. A summary of the previous publications is given in reference [9]. Simi- 
lar findings were made in the study of an imidazolium-based IL $[33,54,55,116]$ and hexaalkylguanidinium-based IL [53] on single-crystalline gold electrodes.

The interpretation of Roling et al. is in accordance with the findings of broadband impedance spectroscopy in two electrode setups ${ }^{1}$. In these experiments electrode polarization effects were measured in an imidazolium-based IL. This polarization effect corresponds to the fast process observed by Roling et al. The decreasing relaxation time with decreasing electrode separation length clearly reveals its diffusive origin $[131,137]$. However, the dependence on the electrode material indicates that this process is not solely diffusion based. The second slower relaxation process is not limited to ILs as shown by a study of a wide range of electrolytes [40]. The authors of this study attribute the slow relaxations to the decreased mobility of ions in the space charge region. However, the $1 \mathrm{D}$ approximations, made in the data analysis of the presented studies, neglect the influence of the finite electrode size. Numerical calculations suggest that electrode edge effects might explain the anomalous effects at low frequencies [104].

Finally, at high frequencies and low temperature the dielectric function can by described by the Dyre model, which describes the charge transport process in glasses by a hoping of charge carriers in an energy landscape [35]. The relaxation times calculated from this model can be related to diffusion coefficients via the Einstein relation and fit well to values obtained from pulsed-field-gradient NMR [130, 131]. Deviations from the Dyre model were observed for high frequencies at very low temperature. This socalled $\beta$-relaxation process has been assigned to librational motions of the imidazolium ring [87].

\section{Electrocapillarity measurements}

Besides modern broadband spectroscopy techniques, the differential capacitance can also be determined with classical electrocapillarity measurements deploying the $\mathrm{Hg}$ drop method. For instance, electrocapillarity measurements were performed on mixtures of imidazolium TFSI-based ILs with different alkyl chain lengths, which show preferential adsorption of long tailed cations[30]. Electrocapillarity curves of various ILs are presented in $[1,92]$. However, one has to be cautious comparing the results from electrocapillarity with other methods. Recent in-situ X-ray reflectivity experiments in aqueous solutions challenge the correctness of this method to determine the absolute surface charge [34].

\subsection{Interfacial structure}

There has been a lot of recent work, in both simulation and experiment, to elucidate the interfacial structure of ILs at hard walls. Interfacial layering structures comprised

\footnotetext{
${ }^{1}$ In this context the technique is called broadband dielectric spectroscopy.
} 


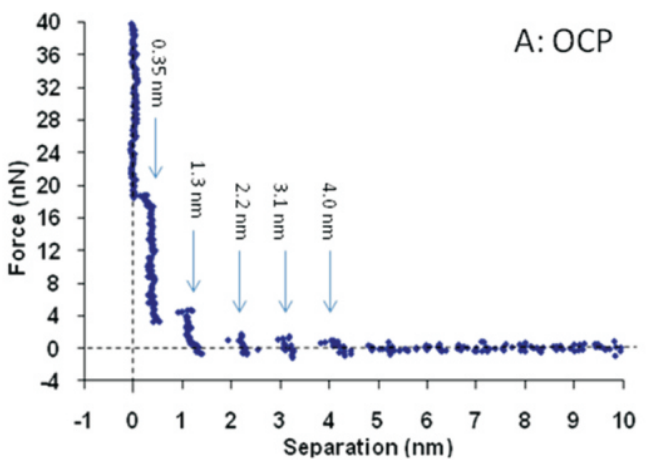

Figure 4.3: Force-distance curve of an AFM tip approaching a $\mathrm{Au}(111)$ electrode in $[\mathrm{bmpy}]^{+}[\mathrm{FAP}]^{-}$at open circuit potential (taken from Hayes et al. [64]).

of cation and anion enriched layers were observed in X-ray reflectivity (XRR) experiments for imidazolium, pyrrolidinium and ammonium-based ILs on various substrates [108, 109, 178]. Also atomic force microscopy (AFM) provided evidence of layers at the interface for several imidazolium and pyrrolidinium-based ILs [10, 32, 176]. A typical force distance curve of an AFM tip penetrating distinct layers while approaching a $\mathrm{Au}(111)$ substrate is shown in figure 4.3. In a recent AFM study the qualitative comparison with MD simulations showed good agreement of force-distance curves and concentration profiles [17]. Though, a quantitative interpretation of the force-distance curves is hampered by the complex interplay between the electrode, the confined IL, and the AFM tip. Furthermore, MD simulation studies confirmed an interfacial structure comprised of alternating cation and anion enriched layers decaying gradually into the bulk [98]. However, these oscillatory correlations at the interface are not necessarily limited to ILs. Also hard sphere fluids may exhibit oscillatory correlations [59, 113], as discussed in chapter 2 .

\section{Experiments under electrochemical control}

The interfacial structure at given electrode potential, i.e. in presence of surface charge, has been investigated both in experiments and in simulations. AFM experiments indicated that the layering is most pronounced at high electrode potential [8, 64]. Especially the first cation layer, adsorbed directly to the electrode, was found to be strongly affected by the electrode potential. Its width and the force to penetrate this layer with the AFM tip strongly depends on the applied voltage $[17,176]$. This finding is in accordance with scanning tunneling microscopy (STM) measurements, revealing distinct adstructures in imidazolium-based ILs on the electrode at elevated potential $[25,54,55,149]$. Also induced molecular reorientation of the adsorbed layer was found by surface sensitive sum frequency generation (SFG) spectroscopy experiments $[2,11,136]$. In these experiments, the orientation of the adsorbed imidazolium ring was found to be dependent on the applied potential. This is similar to findings from MD simulations [99]. Further, attempts were made to study the response of the interfacial structure to electrode potentials by XRR [175] and neutron reflectivity [90]. However, substrate reconstruction on gold surfaces [8] and a limited $q$-range in neutron reflectivity rendered an unambiguous 

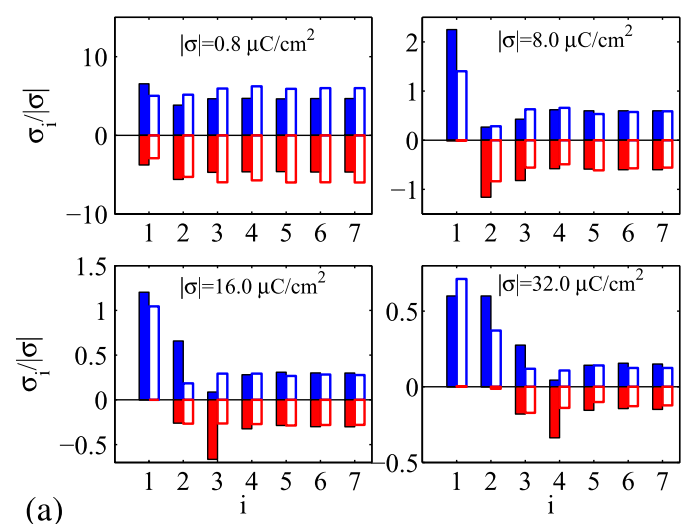

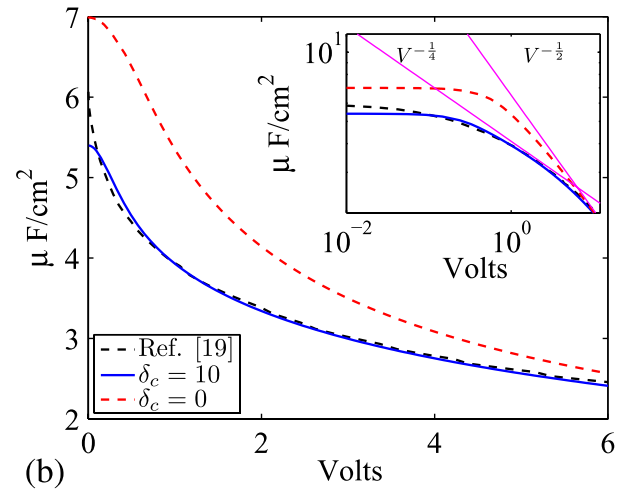

(b)

Figure 4.4: (a) Distribution of anions (red) and cations (blue) sorted into single layers at different surface charges. Solid bars are calculated from the modified PoissonBotzmann equation (3.19) after Bazant et al. while empty bars are derived from MD simulations. (b) Differential capacitance calculated after equation (3.19) (blue line and red dashed line) and MD simulations (dashed black line). The values were derived from the same models as shown in (a) (taken from Bazant et al. [13]).

extraction of the molecular-scale ion structure near the interface impossible.

\section{Differential capacitance in MD simulations}

The interfacial structure determines the relation between the applied potential and the surface charge on the electrode. Thus it is closely related to the differential capacitance (cf. chapter 3). This relation has been studied intensively by MD simulations. Fedorov et al. found interfacial layering structures in MD simulations of simple charged Lennard-Jones spheres at a charged wall [47]. The authors extracted bell-shaped capacitance curves from their simulations which qualitatively correspond to the predictions made by Freise and Kornyshev in their mean-field model. The capacitance values become quantitatively correct if a Stern layer is introduced. In subsequent papers $[46,52]$ deviations from the bell-shaped capacitance are explained by introducing uncharged moieties, representing alkyl tails, which act as "latent voids". This leads to a double peak of the differential capacitance around the potential of zero charge which they call a "camel back"-shaped capacitance. Moreover, the inclusion of dispersion forces can lead to double peaks [162]. In more advanced models of imidazolium- and pyrrolidiniumbased ILs, layering was observed even at a neutral wall [99, 165, 166]. The interfacial structure extended over $20 \AA$ into bulk. Thus, the extended layering structure seems to be more or less independent from the electrode potential which is already screened within the first $1-2 \mathrm{~nm}$. Finally, the found layering structure and the associated capacitance has been reproduced by classical density functional theory of fluids [75, 89]. 


\section{Specific ion adsorption}

Distinct adstructures were observed in imidazolium-based ILs on electrodes by STM. $[25,54,55,149]$. Even at the non-charged electrode a cation enrichment was found in the first layer by MD simulations [99]. This specific adsorption of cations has dramatic effects on the differential capacitance [65] such as a positive potential of zero charge [141]. However, this structured adsorption is not a general characteristic of ILs, as no adstructure was observed in an hexaalkylguanidinium based IL on singlecrystalline $\mathrm{Au}$ electrodes [53]. Furthermore, the surface termination of electrodes influences the adsorbed layer. A surface force apparatus study showed that an imidazolium-based IL exhibits adstructures at mica surfaces but not at hydrocarbon terminated self-assembled monolayer (SAM) surfaces [20].

\section{Interfacial dynamics}

Experimentally, extremely slow dynamics of the interfacial structure were observed upon electrode potential variation. In electrochemical surface plasmon resonance experiments, interfacial relaxation processes on timescales of $100 \mathrm{~s}$ after a potential step were observed in an ammonium-based IL on a polycrystalline gold electrode [112]. The relaxation time in positive direction was found to be two orders of magnitude slower than in negative direction. Similar observations were made in XRR experiments where dynamics on the second scale were found in an imidazolium-based IL [164]. These slow dynamics might be related to the enhanced viscosity of ILs in the interfacial region. Such strong viscosity enhancement and solid like behavior was found by surface force apparatus experiments between charged mica surfaces [20]. Furthermore, both investigated imidazolium-based ILs exhibited pronounced layering. However, in the same study, no such behavior was observed on hydrocarbon terminated SAM surfaces.

Also MD simulations provided evidence for slow dynamics at the interface between ILs and a hard wall. In a study of imidazolium-based ILs, the diffusion coefficient of the first layer next to the interface was found to be reduced to a fraction of the bulk diffusion coefficient $[31,138]$. This diffusion reduction is most pronounced normal to the surface. The adsorbed layer showed an Arrhenius-like behavior. The dynamics of the adsorbed layer were proofed to be very slow, probably solid-like, but simulation times were too short to give clear evidence [143]. 


\section{Part II}

\section{MATERIALS AND METHODS}





\section{Chapter 5}

\section{Material properties}

\subsection{Ionic liquids}

This study aims on the determination of the structure of the IL/electrode interface and its response to applied potentials with a combination of X-ray reflectivity (XRR) and electrochemical methods. For this purpose, ILs are required that are electrochemically stable and that have structures on length scales accessible by XRR experiments on buried interfaces. For this study the ILs 1-butyl-1-methylpyrrolidinium tris(pentafluoroethyl)trifluorophosphate ${ }^{1}[\mathrm{bmpy}]^{+}[\mathrm{FAP}]^{-}$and tetrabutylammonium tris(pentafluoroethyl)trifluorophosphate $[\mathrm{tba}]^{+}[\mathrm{FAP}]^{-}[70]$ have been chosen. Their strong ion-ion correlations result in intense XRD peaks in a $q$-range detectable in XRR. The interfacial structure of both ILs has been sucessfully determined at dielectric sapphire substrates $[109,134]$. These properties makes them the ideal model systems to study electrochemical phenomenons with XRR at the IL/electrode interface. Furthermore, ILs based on the $[\mathrm{FAP}]^{-}$anion are chemically very stable. They have a large electrochemical window, up to $7 \mathrm{~V}$ for the $[\mathrm{tba}]^{+}[\mathrm{FAP}]^{-}$, a superior hydrolytic stability, and a low maximal water uptake which is an order of magnitude smaller compared to ILs with commonly used anions $\left[\mathrm{PF}_{6}\right]^{-}$or $\left[\mathrm{BF}_{4}\right]^{-}$. A maximum water content of less than $10 \mathrm{ppm}$ was achieved in dried liquid [134]. The physio-chemical properties of both ILs is summarized in table 5.1.

The IL $[\mathrm{bmpy}]^{+}[\mathrm{FAP}]^{-}$has been subject of several experimental studies applying different techniques. In AFM experiments, $[\text { bmpy }]^{+}[\mathrm{FAP}]^{-}$showed distinct layering on $\mathrm{Au}(111)$ electrodes (figure 4.3) [8, 41, 64]. At potentials far from the open circuit potential an increased number of layers was observed. The first electrode-adsorbed layer was found to be most affected by the applied potential. Its width and the force needed to penetrate this layer with the AFM tip depends evidently on the applied potential. From these observations the authors concluded that the adsorbed layer is enriched with cations at open circuit potential and at negative potentials while the anion is adsorbed at positive potentials. These findings are affirmed by in situ STM measurements in which island-formation at anodic potential indicates ion exchange [8, 32, 41]. Further,

\footnotetext{
${ }^{1}$ also tris(perfluoroalkyl)trifluorophosphate
} 


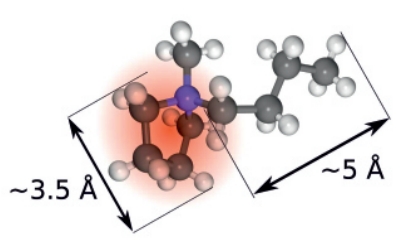

(a) $[\text { bmpy }]^{+}$

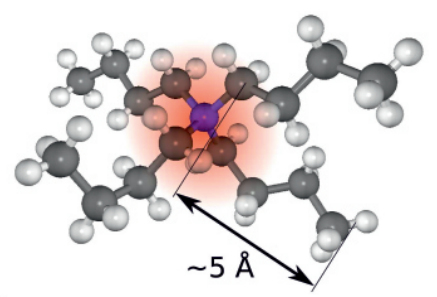

(b) $[\mathrm{tba}]^{+}$

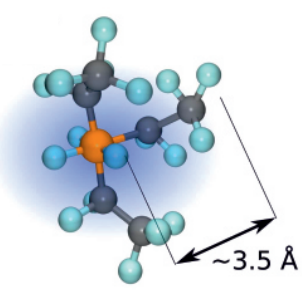

(c) $[\mathrm{FAP}]$

Figure 5.1: Structure of the cations $[\mathrm{bmpy}]^{+}(\mathrm{a})$, [tba $^{+}$(b) and the anion [FAP] ${ }^{-}$(c). Carbon atoms are painted dark gray, hydrogen atoms light gray, nitrogen atoms purple, fluorine light blue, and phosphor orange. Highlighted regions indicate the charged blocks. (taken from Schröder [134]).

Table 5.1: Physio-chemical properties of the studied ILs: anion molecular weight $M_{\mathrm{a}}=$ $445.01 \mathrm{~g} \mathrm{~mol}^{-1}$, cation molecular weight $M_{\mathrm{c}}$, melting temperature $T_{\mathrm{m}}$, crystallization temperature $T_{\mathrm{c}}$, density $\rho_{0}$ at reference temperature $T_{0}$, and linear thermal expansion coefficient $\alpha$.

\begin{tabular}{lcc}
\hline & {$[\mathrm{bmpy}]^{+}[\mathrm{FAP}]^{-}$} & {$[\mathrm{tba}]^{+}[\mathrm{FAP}]^{-}$} \\
\hline$M_{\mathrm{c}}\left(\mathrm{g} \mathrm{mol}^{-1}\right)$ & 142.26 & 242.26 \\
weight ratio c:a & $1: 2$ & $1: 1$ \\
$T_{\mathrm{m}}^{*}\left({ }^{\circ} \mathrm{C}\right)$ & 4 & 70 \\
$T_{\mathrm{c}}^{*}\left({ }^{\circ} \mathrm{C}\right)$ & $\approx-30$ & 40 \\
$\rho_{0}\left(\mathrm{~g} \mathrm{~cm}^{-3}\right)$ & 1.59 & 1.34 \\
$T_{0}\left({ }^{\circ} \mathrm{C}\right)$ & 21 & 72 \\
$\alpha_{\rho}\left(10^{-3} \mathrm{~g} \mathrm{~cm}^{-3} \mathrm{~K}^{-1}\right)$ & 1.0 & 0.8 \\
$\mathrm{RT}$ viscosity $\left(\mathrm{mm} \mathrm{s}^{-2}\right)$ & 184 & $n / a$ \\
electrochem. window $(\mathrm{V})$ & 6.6 & 7.0 \\
max. water uptake $(\mathrm{ppm})$ & $<100$ & $n / a$ \\
\hline
\end{tabular}

${ }^{*}$ at $10 \mathrm{~K} \mathrm{~min}^{-1}$ cf. figure $5.2 \mathrm{a}$ 

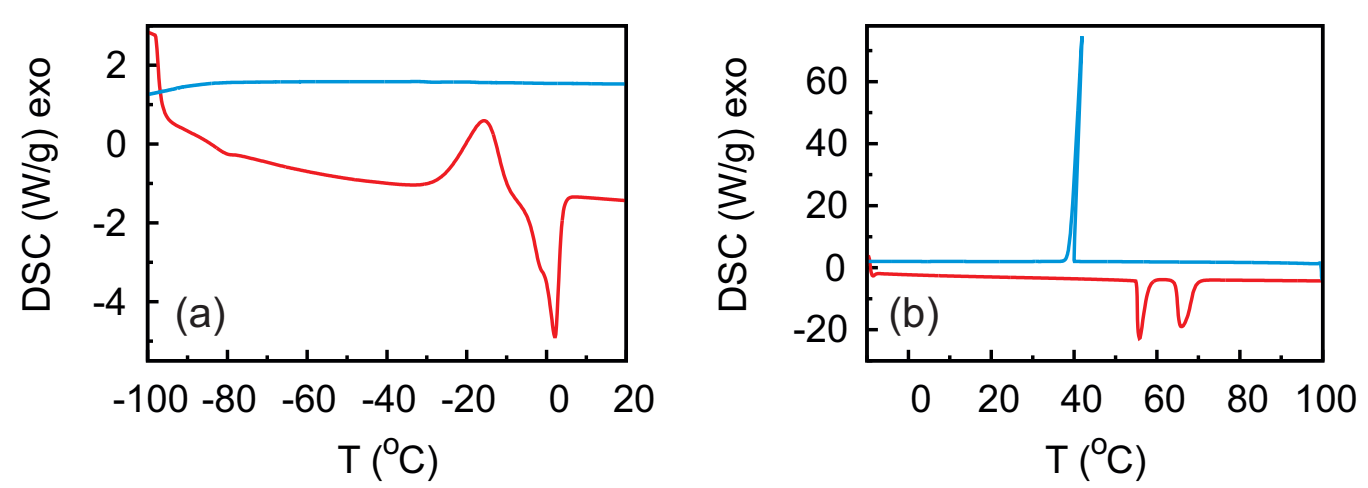

Figure 5.2: DSC data of $[\mathrm{bmpy}]^{+}[\mathrm{FAP}]^{-}$(a) and $[\mathrm{tba}]^{+}[\mathrm{FAP}]^{-}$(b) during cooling (blue line) and heating (red line) with a rate of $10 \mathrm{~K} \mathrm{~min}^{-1}$.

herringbone reconstruction of the $\mathrm{Au}(111)$ surface was observed at cathodic potential. This reconstruction was not seen in imidazolium-based ILs. Thus, the authors speculate that the adsorbed [bmpy] ${ }^{+}$cations might have a templating effect for the reconstruction. The adsorption/desorption process is reversible and happens on the timescale of hours. However, in the described STM measurements no atomic resolution could be achieved for which the authors blame the strongly adsorbed ions. So far, atomic resolution was only achieved in a study of a thin $[\mathrm{bmpy}]^{+}[\mathrm{FAP}]^{-}$layer at an $\mathrm{Au}(111)$ substrate in vaccum at low temperatures [168]. This study showed checkerboard like ordering of the ions in the thin film, also found with gracing incidence X-ray diffraction in a Langmuir film on liquid mercury [150].

Finally, the relaxation dynamics of the interfacial structure was probed by impedance spectroscopy experiments on gold electrodes $[8,32,126]$. Besides the ion diffusion process also much slower processes were observed which were ascribed to interfacial relaxations (cf. section 4.2).

\section{Density and phase transitions}

The temperature dependent mass density $\rho_{\mathrm{m}}(T)$ is given by [134]

$$
\rho_{\mathrm{m}}(T)=\rho_{0}\left[1+\alpha_{\rho}\left(T_{0}-T\right)\right]
$$

where $\rho_{0}$ is the reference mass density at reference temperature $T_{0}$ and $\alpha_{\rho}$ is the coefficient of expansion. The parameters for both ILs are summarized in table 5.1. Further, the melting and crystallization points were determined by differential scanning calorimetry (DSC) (scan rate $10 \mathrm{~K} \mathrm{~min}^{-1}$; DSC-822 Mettler Toledo). The DSC curve in figure $5.2 \mathrm{a}$ shows that $[\mathrm{bmpy}]^{+}[\mathrm{FAP}]^{-}$can be substantially undercooled undergoing a glass transition below $-100^{\circ} \mathrm{C}$. While heating, the ion mobility increases and crystallization starts at about $-30^{\circ} \mathrm{C}$. This is directly followed by the melting process. The melting temperature is $4^{\circ} \mathrm{C}$ which differs considerably from the literature value of below $-50^{\circ} \mathrm{C}[70]$. The crystallization was verified by X-ray diffraction (figure 5.3). 


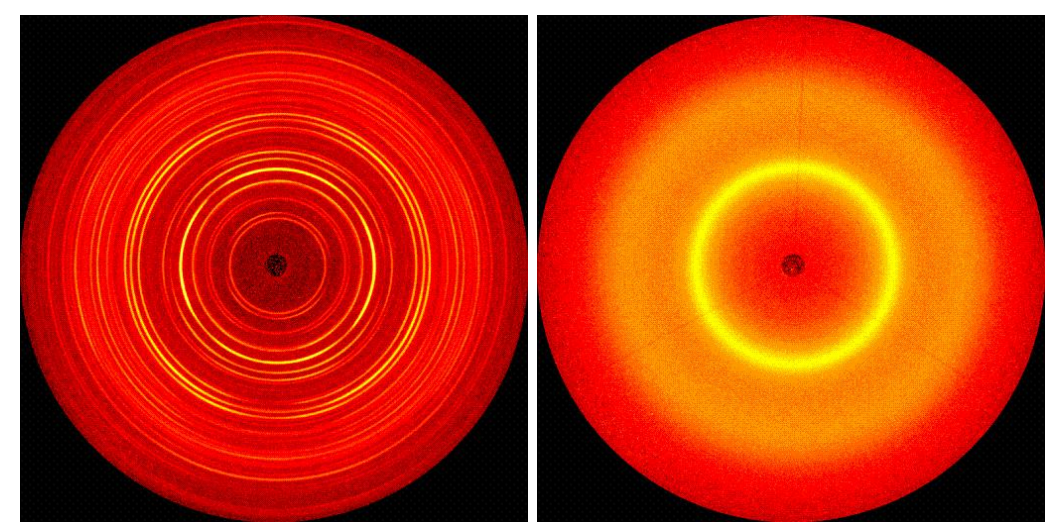

Figure 5.3: XRD images of $[\text { bmpy }]^{+}[\mathrm{FAP}]^{-}$during heating from $-23^{\circ} \mathrm{C}$ (left) to $5{ }^{\circ} \mathrm{C}$ (right).
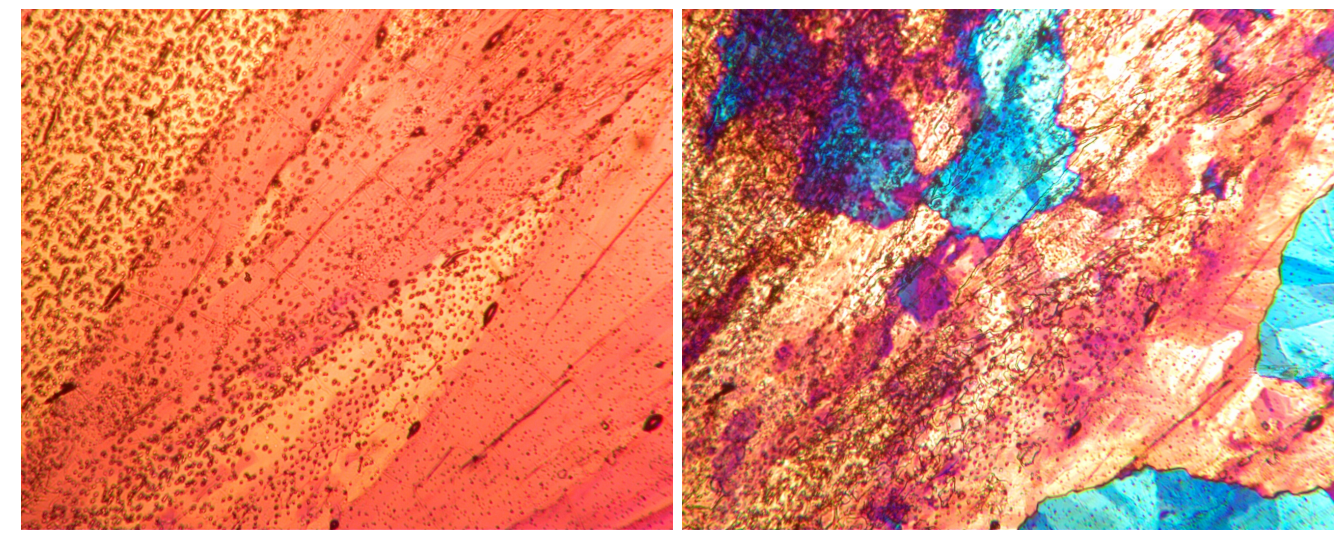

Figure 5.4: Polarized light microscopy images of $[\mathrm{tba}]^{+}[\mathrm{FAP}]^{-}$at $55^{\circ} \mathrm{C}$ (left) and $57^{\circ} \mathrm{C}$ (right).

The second IL $[\mathrm{tba}]^{+}[\mathrm{FAP}]^{-}$can also be undercooled to a temperature of $40{ }^{\circ} \mathrm{C}$ where a crystallization peak appears in the DSC curve (figure 5.2b). The existence of two separate dips while heating indicates two subsequent phase transitions. Indeed, polarized light microscopy images taken below and above the first phase transition temperature reveal a solid-solid phase transition at $56^{\circ} \mathrm{C}$. The melting temperature is found to be $70^{\circ} \mathrm{C}$ which is again different from the literature value of $62^{\circ} \mathrm{C}$ [70]. This kind of solid-solid phase transition is not unusual in ILs and was also observed in imidazoliumbased ILs [36]. The complex molecular structure of the ions and their range of possible conformers imply a complex energetic landscape of the solid state giving rise to unconventional phase transitions.

\section{Maximal interfacial ion excess}

In chapter 3 the lattice gas model was introduced, describing the free energy of an IL at a solid wall. In this model a crucial parameter is the maximal ion excess $\gamma$ at an 
interface. It can be estimated from the molar volume $V_{\mathrm{M}}$ of the ions. For that purpose, equation (3.15) can be written in terms of mass densities

$$
\gamma=\frac{2 \bar{c}}{c_{\max }}=\frac{2 \bar{\rho}_{\mathrm{a} / \mathrm{c}}}{\rho_{\max }^{\mathrm{a} / \mathrm{c}}} .
$$

Here, $\stackrel{\rho}{\mathrm{a} / \mathrm{c}}$ max is the maximal mass density of the adsorbed anion/cation layer and $\bar{\rho}_{\mathrm{a} / \mathrm{c}}$ is the mean mass density of the adsorbed species in bulk IL:

$$
\bar{\rho}_{\mathrm{a} / \mathrm{c}}=\rho_{\mathrm{m}} \frac{M_{\mathrm{a} / \mathrm{c}}}{M_{\mathrm{a}}+M_{\mathrm{c}}} .
$$

with the anion/cation molar mass $M_{\mathrm{a} / \mathrm{c}}$.

$\rho_{\text {max }}$ can be derived from the molecular volume fraction $V_{\mathrm{M}}^{\mathrm{a} / \mathrm{c}}$ of the specific ion species in the IL. First, the molar volume $V_{\mathrm{M}}(T)$ of the IL is calculated from its molar mass $M=M_{\mathrm{a}}+M_{\mathrm{c}}$ and its mass density $\rho_{\mathrm{m}}(T)$ :

$$
V_{\mathrm{M}}(T)=\frac{M_{\mathrm{a}}+M_{\mathrm{c}}}{\rho_{\mathrm{m}}(T)} .
$$

$V_{\mathrm{M}}(T)$ can also be expressed in terms of the effective molar volumes of its constituents $V_{\mathrm{M}}^{\mathrm{a} / \mathrm{c}}[43]$ :

$$
V_{\mathrm{M}}=V_{\mathrm{M}}^{\mathrm{a}}+V_{\mathrm{M}}^{\mathrm{c}} \text {. }
$$

With equation (5.5) and knowing the effective volume of a specific anion allows to calculate the effective molar volumes of a series of cations and anions. Thus, the effective molar volume of a spherical shaped anion is estimated from its ionic radius $r_{\mathrm{a}}$ :

$$
V_{\mathrm{M}}^{\mathrm{a}}=(0.74)^{-1} N_{\mathrm{A}} \frac{3}{4} r_{\mathrm{a}}^{3} \pi .
$$

The prefactor is the volume fraction of closed packed spheres. To validate this method the effective molar volume of the [tba $]^{+}$anion was derived from two different ILs, namely $[\mathrm{tba}]^{+}[\mathrm{Br}]^{-}$and $[\mathrm{tba}]^{+}\left[\mathrm{PF}_{6}\right]^{-}$. The ion radius of $\mathrm{Br}^{-}$yields $1.96 \AA$ [139] while the ion radius of $\left[\mathrm{PF}_{6}\right]^{-}$is $3.08 \AA[43]$. The maximal cation density then yields

$$
\rho_{\max }^{\mathrm{c}}(T)=\frac{M_{\mathrm{c}}}{V_{\mathrm{M}}(T)-V_{M}^{\mathrm{a}}} .
$$

The values of $\rho_{\max }^{\mathrm{a}}$ of the [tba $]^{+}$cation calculated from the two different anion radii is shown in table 5.2. The values deviate less than one percent despite the difference in ion radius and their different composition. Inserting the values from table 5.1 and 5.2 into equations (5.7), (5.3) and (5.2), $\gamma$ can be calculated for the ILs used in this study. The ion excess yields $\gamma=0.80$ for $[\mathrm{bmpy}]^{+}[\mathrm{FAP}]^{-}$and $\gamma=1.15$ for $[\mathrm{tba}]^{+}[\mathrm{FAP}]^{-}$. These values reflects the size ratios between cation and the $[\mathrm{FAP}]^{-}$anion. As $[\mathrm{bmpy}]^{+}$ is smaller than the anion, its maximal volume concentration at the interface is larger than in bulk. The $[\mathrm{tba}]^{+}$cation, on the other hand, is larger than the anion. Therefore, its volume concentration is reduced at the interface. 
Table 5.2: Densities of various ILs $[94,134]$ and the calculated maximal ion densities.

\begin{tabular}{lcccc}
\hline \multicolumn{1}{c}{$\mathrm{IL}$} & $\rho_{\mathrm{m}}\left(\mathrm{g} \mathrm{cm}^{-3}\right)$ & $\rho_{\text {max }}^{\mathrm{a}}\left(\mathrm{g} \mathrm{cm}^{-3}\right)$ & $\rho_{\text {max }}^{\mathrm{c}}\left(\mathrm{g} \mathrm{cm}^{-3}\right)$ & $T\left({ }^{\circ} \mathrm{C}\right)$ \\
\hline$[\mathrm{tba}]^{+}[\mathrm{PF}]^{-}$ & 1.092 & 2.587 & 0.812 & 82 \\
$\mathrm{[tba}^{+}[\mathrm{Br}]^{-}$ & 1.037 & 5.534 & 0.818 & 82 \\
$\mathrm{[tba}^{+}[\mathrm{FAP}]^{-}$ & 1.331 & 2.025 & 0.818 & 82 \\
{$[\mathrm{bmpy}]^{+}[\mathrm{FAP}]^{-}$} & 1.629 & 2.113 & 0.949 & -14 \\
\hline
\end{tabular}

\subsection{Electrode materials}

In situ XRR experiments under potential control require substrates with special properties. They have to be not only chemically stable, but they also have to meet the requirements for high resolution XRR measurements such as evenness and atomic-scale roughness.

\section{Noble metal electrodes}

Potential materials are single crystals of noble metals, like gold or platinum, which can be polished to the desired roughness. Suitable alternatives are thin noble metal films that can be deposited on silicon wafers by physical vapor deposition. Those metal films can be bonded with epoxy resin to a glass substrate. By stripping the metal film together with the glass substrate from the silicon wafer, the superior roughness and evenness of a silicon wafer can be transferred to the noble metal film. Also, liquid mercury exhibits a smooth surface on an atomic-scale, which is suitable for potential controlled XRR experiments [39]. The high surface tension of mercury, however, leads to a high surface curvature of the IL/mercury interface. This curvature has to be minimized by using large sample cells. Therefore, the X-ray beam has to penetrate a long distance through the IL leading to small signals and a huge background from bulk scattering.

Preliminary experimental results and calculations showed that the XRR curve from IL/noble metal interfaces is mostly dominated by the vast difference in electron density between bulk IL and bulk metal. Thus, the signal of the interfacial layering structure is strongly suppressed. In contrast, reflectivity curves from IL/sapphire interfaces showed a clear dip stemming from the oscillatory layering structure [109]. Hence, substrate materials with lower electron densities, like sapphire, are most suitable for extracting information of the interfacial IL structure.

\section{Boron doped diamond}

There are only few low-electron density materials available that possess the desired properties of electrochemical inertness, electrical conductivity and atomic-scale roughness. A material that fulfills all requirements is single-crystalline boron doped diamond (BDD), an electrically conducting material of low electron density, which can be polished below nanometer roughness. CVD boron doped diamonds are commercially avail- 

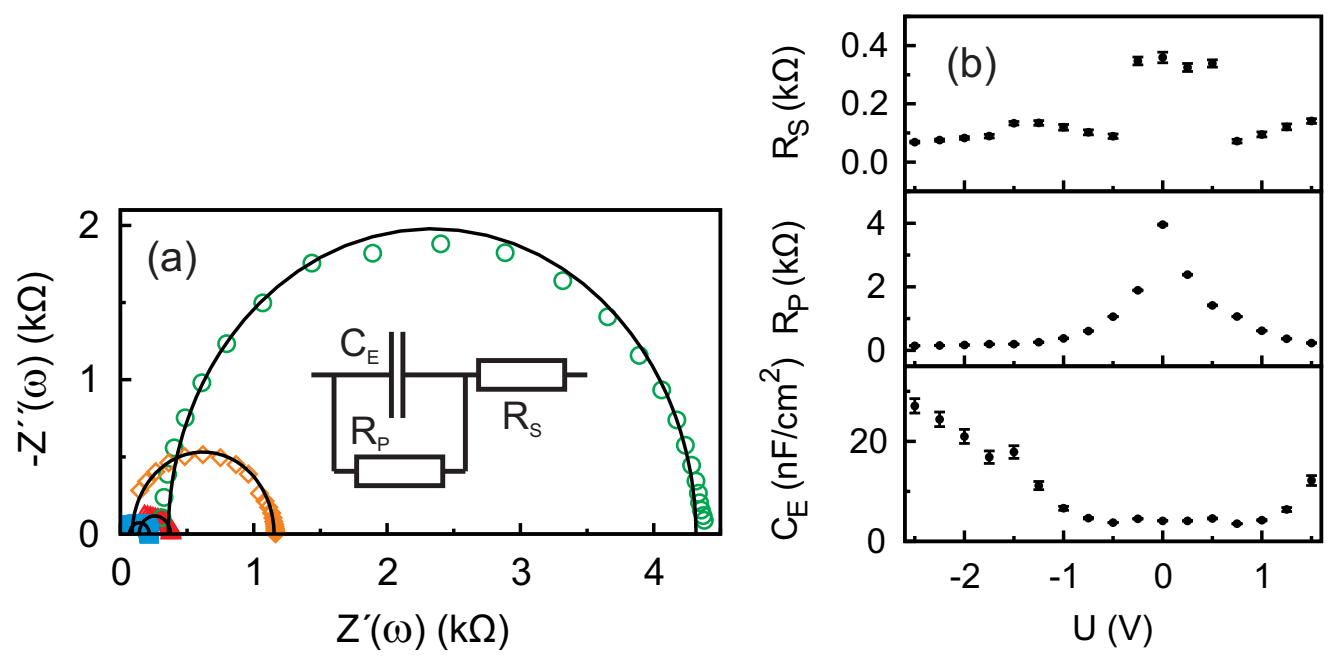

Figure 5.5: (a) Impedance spectra of silver-paint/BDD interface in the complex impedance plane at different bias potentials: $-2.5 \mathrm{~V}$ (blue squares), $-0.5 \mathrm{~V}$ (yellow diamonds), $0 \mathrm{~V}$ (green circles), $1.5 \mathrm{~V}$ (red triangles). The black lines are fits to equations (5.9a) and (5.9b) represented by a equivalent circuit (inset). (b) Fit results.

able specifically for electrochemical applications. Those low-cost highly boron doped electrodes have a very low resistance, below $0.18 \Omega \mathrm{cm}$. However, their polycrystallinity inhibits atomically flat polishing. Therefore, a single-crystalline BDD plate was purchased from the R\&D division of Element Six, Great Britain (table 5.3), which was polished by best effort to sub-nm roughness. Due to lower boron concentration the single crystalline BDD has a higher resistance than the standard boron doped diamond electrodes for electrochemistry purposes. Essentially, its electrical properties are those of a doped semiconductor.

The electrical properties of the BDD were characterized by impedance spectroscopy measurements (cf. chapter 7). The BDD was bonded with silver paint between metal electrodes and impedance spectra were recorded for bias potentials ranging from $-2.5 \mathrm{~V}$ to $1.5 \mathrm{~V}$. The impedance spectra were fitted to an equivalent circuit consisting of a resistor $R_{\mathrm{S}}$ in series with a parallel circuit of a capacitance $C_{\mathrm{E}}$ and a resistor $R_{\mathrm{P}}$ (inset figure 5.5). This equivalent circuit yields a complex impedance of

$$
\hat{Z}(\omega)=R_{\mathrm{S}}+\frac{R_{\mathrm{P}}}{1+i \omega R_{\mathrm{P}} C_{\mathrm{E}}},
$$

which can be separated in its real part $Z^{\prime}(\omega)$ and imaginary part $Z^{\prime \prime}(\omega)$ :

$$
\begin{gathered}
Z^{\prime}(\omega)=R_{\mathrm{S}}+\frac{R_{\mathrm{P}}}{1+\left(\omega R_{\mathrm{P}} C_{\mathrm{E}}\right)^{2}} \\
Z^{\prime \prime}(\omega)=\frac{\omega C_{\mathrm{E}} R_{\mathrm{P}}^{2}}{1+\left(\omega R_{\mathrm{P}} C_{\mathrm{E}}\right)^{2}}
\end{gathered}
$$


The fits to equations (5.9a) and (5.9b) at different bias potentials are shown in figure 5.5a. The resulting capacitance and resistance show the typical potential dependence of a series of metal-semiconductor Schottky junctions (figure 5.5b), formed of the two silver paint/BDD interfaces. At zero bias potential the overall resistance $R_{\mathrm{S}}+R_{\mathrm{P}} \approx$ $4.5 \mathrm{k} \Omega$ is relatively high. For bias potentials larger than $0.5 \mathrm{~V}$, the charge carriers can easily pass the Schottky barrier at the metal/semiconductor interface and the resistance decreases to about $0.2 \mathrm{k} \Omega$. Also the capacitance shows a typical potential dependence: Because of the relatively low charge carrier density, the surface charge is not situated directly at the interface as compared to metal electrodes, but it is distributed in a spacecharge region. It shows a similar parabolic potential dependence as the capacitance of a dilute solution (cf. chapter 3).

Table 5.3: Physical properties of the singlecrystalline boron doped diamond

\begin{tabular}{|c|c|}
\hline $\operatorname{size}^{*}\left(\mathrm{~mm}^{-3}\right)$ & $4 \times 4 \times 0.3$ \\
\hline boron concentration* $\left(\mathrm{cm}^{-3}\right)$ & $10^{19}$ \\
\hline resistivity* $(\Omega \mathrm{cm})$ & $<20$ \\
\hline resistivity at $0 \mathrm{~V}(\Omega \mathrm{cm})$ & 24000 \\
\hline resistivity at $-2.5 \mathrm{~V}(\Omega \mathrm{cm})$ & 1000 \\
\hline $\operatorname{misscut}^{*}\left({ }^{\circ}\right)$ & $<3$ \\
\hline orientation faces* & $(100)$ \\
\hline orientation edges* & $\langle 110\rangle$ \\
\hline roughness* (best effort) $(\AA)$ & $<10$ \\
\hline
\end{tabular}

* information provided by supplier

\section{Reference electrodes}

In order to measure potentials in non-aqueous solutions, such as ILs, quasi reference electrodes are often used $[12,74]$. These electrodes consist of noble metals e.g. platinum or silver. In contrast to redox-based reference electrodes, such as $\mathrm{Ag} / \mathrm{Ag}^{+}$, those electrodes do not have a fixed potential difference to the standard hydrogen electrode potential. Thus, the potential is calibrated in each experiment by adding a substance, which has a reproducible redox potential. However, potential drifts versus the standard hydrogen electrode potential can occur over time. For instance, in an imidazolium-based IL, the potential was found to vary $\pm 5 \mathrm{mV}$ over several weeks [67]. In this experiment the reference electrode was separated from electroactive compounds by a glass frit.

In the presented study the platinum quasi reference electrode was directly immersed in the IL because of design constraints (cf. figure 8.1). This arrangement can lead to potential drifts of several $100 \mathrm{mV}$ [66]. In order to determine the approximate potential vs. standard electrode, a cyclic voltammetry (CV) measurement was performed on a solution of $[\mathrm{tba}]^{+}[\mathrm{FAP}]^{-}$and the calibrant ferrocene, which has a strong peak at $0.31 \mathrm{~V}$ 


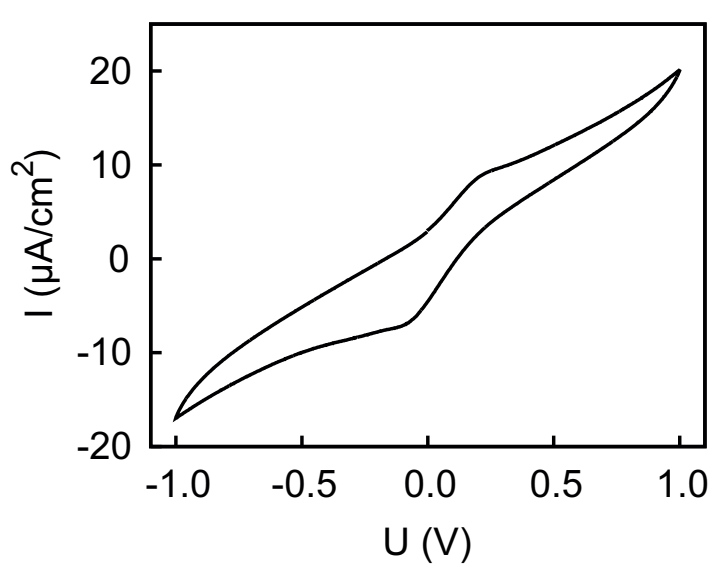

Figure 5.6: $\mathrm{CV}$ curve of ferrocene in $[\mathrm{tba}]^{+}[\mathrm{FAP}]^{-}$using a platinum quasi reference electrode with a cycling speed of $100 \mathrm{mV} \mathrm{s}^{-1}$ at $83^{\circ} \mathrm{C}$ under nitrogen atmosphere.

versus $\mathrm{SCE}^{2}$ [12]. Figure 5.6 shows the respective $\mathrm{CV}$ recorded with a cycling speed of $100 \mathrm{mV} \mathrm{s}^{-1}$ at $83^{\circ} \mathrm{C}$ under nitrogen atmosphere. The oxidation and reduction peaks are quite weak compared to reported data [66]. One reason might be the generally poor solubility of ferrocene in ILs [74]. Though, the half-wave potential ${ }^{3}$ could be estimated to approx. $0.08 \mathrm{~V}$. Basically, this calibration has to be done after each electrochemical experiment. However, to do so, the experimental chamber has to be opened and the working electrode has to be removed. Such a calibration procedure would be questionable due to possible contamination. Therefore, all measured potentials in this study are given versus platinum quasi reference electrode and not standard electrode potential.

\footnotetext{
${ }^{2} \mathrm{Hg} / \mathrm{Hg}^{+}$standard calomel electrode: $0.242 \mathrm{~V}$ versus normal hydrogen electrode

${ }^{3}$ mean potential of the oxidation and reduction peak
} 


\section{Chapter 6}

\section{X-ray scattering}

$\mathrm{X}$-rays are electromagnetic waves with wavelengths in the region of $10^{-10} \mathrm{~m}=1 \AA$, i.e. on atomic length-scales. Since their discovery by Wilhelm Conrad Röntgen in 1895, Xrays became an invaluable tool to unravel the atomic structure of matter. With the advent of synchrotron sources in the 70s and the progressive increase in intensity by a factor of $10^{12}$ compared to the first sources, a myriad of new techniques emerged and X-rays became a standard tool in material science. In this chapter the fundamentals of X-ray scattering are briefly introduced and applied to the problem of scattering from liquid structures. Then, the phenomenological Teubner-Strey model for long-range correlations in liquids is presented. Afterwards, the special geometry of X-ray reflectivity will be described which is a powerful technique to investigate surface and buried interfacial profiles. Finally, a modified distorted crystal model is presented describing the IL/solid interface. A more comprehensive treatment of X-ray scattering and X-ray reflectivity can be found in [3, 159]. A rigorous discussion of liquid structures is presented in [62].

\section{Scattering from one free electron}

The interaction of X-rays with matter is described by the scattering formalism. A fundamental quantity in all scattering experiments is the differential scattering cross-section

$$
\left(\frac{\mathrm{d} \sigma}{\mathrm{d} \Omega}\right)=\frac{I^{\mathrm{S}}}{\Phi_{0} \Delta \Omega} .
$$

It describes the intensity $I^{\mathrm{S}}$ scattered into the solid angle $\Delta \Omega$ divided by the incident $\mathrm{X}$ ray flux $\Phi_{0}$. For X-rays scattered by a free electron, $(\mathrm{d} \sigma / \mathrm{d} \Omega)$ is given by the Thomson differential cross-section

$$
\left(\frac{\mathrm{d} \sigma}{\mathrm{d} \Omega}\right)=r_{\mathrm{e}}^{2}\left|\varepsilon \cdot \varepsilon^{\prime}\right|^{2}
$$

Equation (6.2) can be derived by means of classical electric dipole radiation, but also from a full quantum mechanical treatment [3]. Here, $r_{\mathrm{e}}=2.82 \times 10^{-5} \AA$ is the Thomson scattering length of the electron, also known as the classical electron radius. As 
Figure 6.1: Scattering geometry of an incident X-ray beam with wave vector $\mathbf{k}_{\mathrm{i}}$ and scattered wave vector $\mathbf{k}_{\mathrm{f}}$ into the solid angle $\Delta \Omega$. The scattering angle $2 \theta$ between $\mathbf{k}_{\mathrm{i}}$ and $\mathbf{k}_{\mathrm{f}}$ corresponds to a scatter-

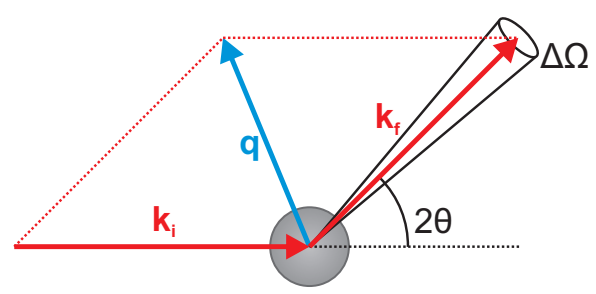
ing vector of $\mathbf{q}=\mathbf{k}_{\mathrm{f}}-\mathbf{k}_{\mathrm{i}}$.

X-rays are transverse waves, the scattered intensity depends on the polarization of the incident $\varepsilon$ and the scattered wave $\varepsilon^{\prime}$ [3]:

$$
\left|\varepsilon \cdot \varepsilon^{\prime}\right|^{2}= \begin{cases}1 & \text { vertical scattering plane (synchrotron) } \\ \cos ^{2}(2 \theta) & \text { horizontal scattering plane (synchrotron) } \\ \frac{1}{2}\left[1+\cos ^{2}(2 \theta)\right] & \text { unpolarized source (e.g. X-ray tube) }\end{cases}
$$

\section{Scattering from one atom}

In matter electrons are usually bound to atoms. Thus, the electron cannot be regarded as a point like particle anymore but as a diffuse charge distribution $\rho_{\text {at }}(\mathbf{r})$ around the atomic nucleus. This leads to an angular dependent cross-section. The scattering process can be described in a typical scattering geometry (figure 6.1). Here, we introduce the wave vector $\mathbf{k}=\frac{2 \pi}{\lambda} \mathbf{e}$ in direction $\mathbf{e}=\mathbf{k} /|\mathbf{k}|$. For electromagnetic waves the wavelength $\lambda$ is related to the photon energy by $\lambda=12.4 \AA / E[\mathrm{keV}]$. The difference between the incident wave vector $\mathrm{k}_{\mathrm{i}}$ and the scattered wave vector $\mathbf{k}_{\mathrm{f}}$ is called wave vector transfer, momentum transfer or scattering vector $\mathbf{q}=\mathbf{k}_{\mathrm{f}}-\mathbf{k}_{\mathrm{i}}$. In the case of elastic scattering, i.e. no energy transfer between sample and photon, the absolute value of the wave vector is constant, i.e. $\left|\mathbf{k}_{\mathrm{f}}\right|=\left|\mathbf{k}_{\mathrm{i}}\right|=k$, and the scattering vector yields

$$
q=2 k \sin \theta=\frac{4 \pi}{\lambda} \sin \theta .
$$

The differential cross-section of an atom reads

$$
\left(\frac{\mathrm{d} \sigma}{\mathrm{d} \Omega}\right)=r_{\mathrm{e}}^{2}\left|\varepsilon \cdot \varepsilon^{\prime}\right|^{2}|f(\mathbf{q}, \omega)|^{2} .
$$

The atomic form factor $f(\mathbf{q}, \omega)$ describes the deviation from the Thomson scattering of a free electron and depends on the scattering vector $\mathbf{q}$ and the energy $\hbar \omega$ of the X-rays. $f(\mathbf{q}, \omega)$ can be separated in an angular dependent and energy dependent part:

$$
f(\mathbf{q}, \omega)=f^{0}(\mathbf{q})+f^{\prime}(\omega)+i f^{\prime \prime}(\omega)
$$

The angular dependent part $f^{0}(\mathbf{q})$ is equal to the Fourier transform of the electron density distribution $\rho_{\text {at }}(\mathbf{r})$ of the atom

$$
f^{0}(\mathbf{q})=\int \rho_{\mathrm{at}}(\mathbf{r}) \mathrm{e}^{-i \mathbf{q r}} \mathrm{d} \mathbf{r}
$$


The energy dependent terms $f^{\prime}(\omega)$ and $f^{\prime \prime}(\omega)$ are called dispersion corrections. Their origin can be understood by regarding the bound electrons as damped harmonic oscillators with resonance frequency $\omega_{\mathrm{s}}$. Their contribution is significant at X-ray energies of about $\hbar \omega_{\mathrm{s}}$, leading to the well known absorption edges. In this thesis only high energy $\mathrm{X}$-rays are used with energies well above all absorption edges of the studied materials. Therefore, the dispersion corrections will be neglected in the following and the form factor is written

$$
f(\mathbf{q}, \omega) \approx f^{0}(\mathbf{q}) \stackrel{!}{=} f(\mathbf{q})
$$

\section{Compton scattering}

Until now, only elastic scattering was considered. However, the incident X-rays can also be regarded as particles, i.e. photons. Thus, collisions between photons and electrons will lead to energy transfer, i.e. inelastic scattering. This process is called Compton scattering and cannot be described by classical electrodynamics. For the scattering of an $\mathrm{X}$-ray photon and a free electron the following relation can be derived from relativistic energy conservation:

$$
\frac{\left|\mathbf{k}_{\mathrm{i}}\right|}{\left|\mathbf{k}_{\mathrm{f}}\right|}=1+\lambda_{\mathrm{C}}\left|\mathbf{k}_{\mathrm{i}}\right|(1-\cos 2 \theta)
$$

with the Compton scattering length $\lambda_{\mathrm{C}}=\hbar /\left(m_{\mathrm{e}} c\right)=3.86 \times 10^{-3} \AA$. Compton scattering is incoherent in contrast to Thomson scattering. Thus, no interference effects occur and the scattering is, in first order, independent of the electron arrangement. It gives rise to a broad background scattering, which can be subtracted from the total scattered intensity.

\subsection{The liquid structure factor}

The derivation of the liquid structure factor presented in this section follows [62] with particular extension for X-ray scattering [3].

In most elastic neutron and X-ray experiments on bulk amorphous samples, the scattered intensities are weak, if the sample thickness is about the inverse of the absorption length. Weak means that the incident X-rays or neutrons are only scattered once and that the scattered wave can be regarded as a spherical elementary wave. Therefore, the kinematic approximation ${ }^{1}$ can be used and the differential cross-section ${ }^{2}(\mathrm{~d} \sigma / \mathrm{d} \Omega)$ at scattering angle $q$ is equal to the square of the Fourier transform of the scattering density $\hat{\rho}(\mathbf{q})$ :

$$
\left(\frac{\mathrm{d} \sigma}{\mathrm{d} \Omega}\right)=\left\langle|\hat{\rho}(\mathbf{q})|^{2}\right\rangle=\left\langle\hat{\rho}(\mathbf{q}) \hat{\rho}^{*}(\mathbf{q})\right\rangle
$$

\footnotetext{
${ }^{1}$ In quantum mechanics the kinetic approximation is also called Born approximation

${ }^{2}$ For clarity the differential cross-section is given in electron units $r_{\mathrm{e}}^{2}$ and the polarization factor (6.3) is set to 1
} 


$$
\hat{\rho}(\mathbf{q})=\int \rho(\mathbf{r}) \mathrm{e}^{-i \mathbf{q r}} \mathrm{d} \mathbf{r} .
$$

In equation (6.10a) a thermodynamic average \langle\rangle over an ensemble of micro states is applied. This average is valid for scattering experiments in which the beam size $(\mu \mathrm{m}-\mathrm{mm})$ is usually much larger than the characteristic length scale of the system. Also, the integration time in a typical scattering experiment is usually much longer than molecular diffusion times. In the simple case of a mono-atomic or mono-molecular liquid, the real space scattering density can be written as a sum of the scattering densities $\rho_{\text {at }}(\mathbf{r})$ of all single atoms at positions $\mathbf{r}_{n}$ :

$$
\rho(\mathbf{r})=\sum_{n} \rho_{\mathrm{at}}\left(\mathbf{r}-\mathbf{r}_{n}\right)
$$

With equation (6.11) the Fourier transform of the scattering density (6.10b) can be written as

$$
\begin{gathered}
\hat{\rho}(\mathbf{q})=\sum_{n} f(\mathbf{q}) \mathrm{e}^{-i \mathbf{q} \mathbf{r}_{n}} \\
f(\mathbf{q})=\int \rho_{\mathrm{at}}(\mathbf{r}) \mathrm{e}^{-i \mathbf{q r}} \mathrm{d} \mathbf{r}
\end{gathered}
$$

with the atomic form factor $f(\mathbf{q})^{3}$. Then, the differential cross-section (6.10a) reads

$$
\left(\frac{\mathrm{d} \sigma}{\mathrm{d} \Omega}\right)=\left\langle|\hat{\rho}(\mathbf{q})|^{2}\right\rangle=f(\mathbf{q})^{2}\left\langle\sum_{n} \mathrm{e}^{-i \mathbf{q} \mathbf{r}_{n}} \sum_{m} \mathrm{e}^{i \mathbf{q} \mathbf{r}_{m}}\right\rangle .
$$

Now the scattering amplitude of the single atom can be separated from the the amplitude originating from the spacial arrangement of the atoms by defining the structure factor $S_{\mathrm{m}}(\mathbf{q})$ of a mono-atomic liquid

$$
\left(\frac{\mathrm{d} \sigma}{\mathrm{d} \Omega}\right)=N f(\mathbf{q})^{2} S_{\mathrm{m}}(\mathbf{q}) .
$$

The structure factor can be rewritten:

$$
\begin{aligned}
& S_{\mathrm{m}}(\mathbf{q})=\left\langle\frac{1}{N} \sum_{n} \mathrm{e}^{-i \mathbf{q} \mathbf{r}_{n}} \sum_{m} \mathrm{e}^{i \mathbf{q} \mathbf{r}_{m}}\right\rangle \\
& =\left\langle\frac{1}{N} \sum_{n} \sum_{m} \mathrm{e}^{-i \mathbf{q}\left(\mathbf{r}_{n}-\mathbf{r}_{m}\right)}\right\rangle \\
& =1+\left\langle\frac{1}{N} \sum_{n} \sum_{m \neq n} \mathrm{e}^{-i \mathbf{q}\left(\mathbf{r}_{n}-\mathbf{r}_{m}\right)}\right\rangle \\
& =1+\left\langle\frac{1}{N} \sum_{n} \sum_{m \neq n} \iint \mathrm{e}^{-i \mathbf{q}\left(\mathbf{r}-\mathbf{r}^{\prime}\right)} \delta\left(\mathbf{r}-\mathbf{r}_{n}\right) \delta\left(\mathbf{r}^{\prime}-\mathbf{r}_{m}\right) \mathrm{d} \mathbf{r} \mathrm{d} \mathbf{r}^{\prime}\right\rangle \\
& =1+\frac{1}{N} \iint \bar{\rho} g\left(\mathbf{r}-\mathbf{r}^{\prime}\right) \mathrm{e}^{-i \mathbf{q}\left(\mathbf{r}-\mathbf{r}^{\prime}\right)} \mathrm{d} \mathbf{r} \mathrm{d} \mathbf{r}^{\prime} .
\end{aligned}
$$

\footnotetext{
${ }^{3} f(\mathbf{q})$ is equal to $f^{0}(\mathbf{q})$ in equation (6.7) neglecting the dispersion corrections
} 
In the last step, the mean particle density $\bar{\rho}$ and the pair distribution function $g\left(\mathbf{r}-\mathbf{r}^{\prime}\right)$ were introduced:

$$
\bar{\rho} g\left(\mathbf{r}-\mathbf{r}^{\prime}\right)=\left\langle\sum_{n} \sum_{n \neq m} \delta\left(\mathbf{r}-\mathbf{r}_{n}\right) \delta\left(\mathbf{r}^{\prime}-\mathbf{r}_{m}\right)\right\rangle .
$$

The pair distribution function $g\left(\mathbf{r}-\mathbf{r}^{\prime}\right)$ states the probability of finding a second particle at a distance $\mathbf{r}-\mathbf{r}^{\prime}$ from a test particle (cf. chapter 2). As only the difference of the scattering density from the mean density contributes to the signal, we add and subtract a term to $S_{\mathrm{m}}(\mathbf{q})$ corresponding to the local mean scattering density ${ }^{4}$ :

$$
\begin{aligned}
S_{\mathrm{m}}(\mathbf{q})=1 & +\frac{\bar{\rho}}{N} \iint\left(g\left(\mathbf{r}-\mathbf{r}^{\prime}\right)-1\right) \mathrm{e}^{-i \mathbf{q}\left(\mathbf{r}-\mathbf{r}^{\prime}\right)} \mathrm{d} \mathbf{r} \mathrm{d} \mathbf{r}^{\prime} \\
& +\frac{\bar{\rho}}{N} \iint \mathrm{e}^{-i \mathbf{q}\left(\mathbf{r}-\mathbf{r}^{\prime}\right)} \mathrm{d} \mathbf{r} \mathrm{d} \mathbf{r}^{\prime}
\end{aligned}
$$

The last term describes scattering density variations on large length-scales and is only present at small q. This term contributes to the prominent $\mathrm{SAXS}^{5}$ signal, but can be omitted for higher q vectors. As liquids are isotropic, their structure is invariant under translations and rotations. Thus, one of the integrals over the whole volume yields the number of scatterers $N$ and the second integral can be rewritten as an radial integral, i.e. $\mathrm{e}^{-i \mathbf{q r}} \mathrm{d} \mathbf{r} \rightarrow 4 \pi r^{2} \sin (q r) /(q r) \mathrm{d} r$. With this, $S_{\mathrm{m}}(q)$ can be written in terms of the radial distribution function $g(r)$ :

$$
S_{\mathrm{m}}(q)=1+\bar{\rho} \int(g(r)-1) 4 \pi r^{2} \frac{\sin (q r)}{q r} \mathrm{~d} r .
$$

With the definition of the total correlation function $h(r)=g(r)-1$ (cf. chapter 2), the final result for the mono-atomic liquid structure factor yields

$$
S_{\mathrm{m}}(q)=1+\frac{4 \pi \bar{\rho}}{q} \int_{0}^{\infty} h(r) r \sin (q r) \mathrm{d} r .
$$

\section{Polyatomic systems}

For polyatomic systems the structure factor can be divided into partial structure factors describing the correlations between different atomic species. The partial structure factors can be derived in analogy to the monoatomic structure factor, as demonstrated in the following. If only correlations up to the second order are considered, the differential

\footnotetext{
${ }^{4}$ This is a didactic derivation often used in textbooks [3]. $\bar{\rho}$ is not really a constant, but its variation is small. Thus the large-scale scattering density variations can be separated from the fluctuations on molecular length-scales.

${ }^{5}$ small angle $\mathrm{X}$-ray scattering
} 
cross-section can be written as [62]:

$$
\begin{aligned}
\left(\frac{\mathrm{d} \sigma}{\mathrm{d} \Omega}\right) & =\left\langle\hat{\rho}_{\alpha}(\mathbf{q}) \hat{\rho}_{\beta}^{*}(\mathbf{q})\right\rangle \\
& =\left\langle\sum_{\alpha, n} f_{\alpha}(\mathbf{q}) \mathrm{e}^{-i \mathbf{q} \mathbf{r}_{n \alpha}} \sum_{\beta, m} f_{\beta}^{*}(\mathbf{q}) \mathrm{e}^{i \mathbf{q} \mathbf{r}_{m \beta}}\right\rangle
\end{aligned}
$$

where the sum over $\alpha$ and $\beta$ is taken over all atomic species. The self scattering term can be separated from the mixed term:

$$
\begin{aligned}
\left(\frac{\mathrm{d} \sigma}{\mathrm{d} \Omega}\right)= & N \sum_{\alpha} c_{\alpha} f_{\alpha}(\mathbf{q}) f_{\alpha}^{*}(\mathbf{q})+\left\langle\sum_{\alpha, \beta} \sum_{n, m \neq n} f_{\alpha}(\mathbf{q}) f_{\beta}^{*}(\mathbf{q}) \mathrm{e}^{i \mathbf{q}\left(\mathbf{r}_{m \beta}-\mathbf{r}_{n \alpha}\right)}\right\rangle \\
= & f_{\alpha}(\mathbf{q}) f_{\beta}^{*}(\mathbf{q})\left(N \sum_{\alpha} c_{\alpha} \delta_{\alpha \beta}+\right. \\
& \left.\left\langle\sum_{\alpha, \beta} \sum_{n} \sum_{m \neq n} \int \mathrm{e}^{-i \mathbf{q r}} \delta\left(\mathbf{r}+\mathbf{r}_{n \alpha}-\mathbf{r}_{m \beta}\right) \mathrm{d} \mathbf{r}\right\rangle\right) \\
= & N f_{\alpha}(\mathbf{q}) f_{\beta}^{*}(\mathbf{q})\left(\sum_{\alpha} c_{\alpha} \delta_{\alpha \beta}+\sum_{\alpha, \beta} c_{\alpha} c_{\beta} \bar{\rho} \int g_{\alpha \beta}(\mathbf{r}) \mathrm{e}^{-i \mathbf{q r}} \mathrm{d} \mathbf{r}\right) .
\end{aligned}
$$

In the first step the translation symmetry of the system is used and the origin is arbitrarily set to zero. In the second step the partial pair distribution function $g_{\alpha \beta}(\mathbf{r})$ was introduced:

$$
c_{\alpha} c_{\beta} \bar{\rho} g_{\alpha \beta}(\mathbf{r})=\left\langle\frac{1}{N} \sum_{n} \sum_{n \neq m} \delta\left(\mathbf{r}+\mathbf{r}_{n \alpha}-\mathbf{r}_{m \beta}\right)\right\rangle .
$$

$g_{\alpha \beta}(\mathbf{r})$ states the probability of finding an atom of species $\beta$ at a distance $\mathbf{r}$ from an atom of species $\alpha$. As liquids are isotropic one can apply an angular average on the integral over the sample volume, i.e. $\mathrm{e}^{-i \mathbf{q r}} \mathrm{d} \mathbf{r} \rightarrow 4 \pi r^{2} \sin (q r) /(q r) \mathrm{d} r$. Omitting long-range density variations in analogy to the mono-atomic case, only differences of the scattering density from the mean density contribute to the scattered signal, i.e.

$$
g_{\alpha \beta}(r) \rightarrow\left(g_{\alpha \beta}(r)-1\right)=h_{\alpha \beta}(r) .
$$

Here the partial total correlation function $h_{\alpha \beta}(r)$ (2.5) was introduced. Thus, the differential cross section (6.21) can be rewritten:

$$
\left(\frac{\mathrm{d} \sigma}{\mathrm{d} \Omega}\right)=N f_{\alpha}(q) f_{\beta}^{*}(q)\left(\sum_{\alpha} c_{\alpha} \delta_{\alpha \beta}+\sum_{\alpha, \beta} c_{\alpha} c_{\beta} \frac{4 \pi \bar{\rho}}{q} \int_{0}^{\infty} h_{\alpha \beta}(r) r \sin (q r) \mathrm{d} r\right) .
$$

With the definition of the partial structure factor after Pings and Waser [118]

$$
S_{\alpha \beta}(q)=\frac{c_{\alpha} c_{\beta} f_{\alpha}(q) f_{\beta}^{*}(q) \frac{4 \pi \bar{\rho}}{q} \int_{0}^{\infty} h_{\alpha \beta}(r) r \sin (q r) \mathrm{d} r}{\left|\sum_{\alpha} c_{\alpha} f_{\alpha}(q)\right|^{2}}
$$


the differential cross section finally reads

$$
\left(\frac{\mathrm{d} \sigma}{\mathrm{d} \Omega}\right)=N \sum_{\alpha} c_{\alpha}\left|f_{\alpha}(q)\right|^{2}+N\left|\sum_{\alpha} c_{\alpha} f_{\alpha}(q)\right|^{2} \sum_{\alpha, \beta} S_{\alpha \beta}(q) .
$$

This definition is most suitable for X-ray scattering. In common X-ray experiments it cannot be distinguished easily between the different atomic species and thus, the partial structure factors are not accessible. The partial structure factors can only be determined indirectly by advanced simulation-aided data analysis as described in chapter 4 . However, X-ray scattering experiments are still sensitive to the total scattering density variation of all electrons. Consequently, a total structure factor $S(q)$ can be defined for which the differential scattering cross section yields

$$
\begin{gathered}
\left(\frac{\mathrm{d} \sigma}{\mathrm{d} \Omega}\right)=N \sum_{\alpha} c_{\alpha}\left|f_{\alpha}(q)\right|^{2}+N\left|\sum_{\alpha} c_{\alpha} f_{\alpha}(q)\right|^{2} S(q) \\
S(q)=\sum_{\alpha, \beta} S_{\alpha \beta}(q) .
\end{gathered}
$$

In contrast to the mono-atomic structure factor (6.19), $S(q)$ does not include the selfscattering term. In the presence of more than one atom species, the atomic form factors cannot easily be separated from the structure factor anymore. This separation of self scattering and inter-atomic scattering is the reason why the definition of the partial structure factor (6.25) is advantageous for X-ray scattering. Other definitions used in different scientific communities are presented below.

Real space information is accessible via the total pair correlation function $h(r)=$ $g(r)-1$ which can be directly extracted from the total structure factor $S(q)$

$$
\begin{gathered}
S(q)=\frac{4 \pi \bar{\rho}}{q} \int_{0}^{\infty} h(r) r \sin (q r) \mathrm{d} r \\
h(r)=\sum_{\alpha, \beta} U_{\alpha \beta} h_{\alpha \beta}(r) .
\end{gathered}
$$

The transformation matrix

$$
U_{\alpha \beta}=\frac{c_{\alpha} c_{\beta} \int_{0}^{\infty} f_{\alpha}(q) f_{\beta}^{*}(q) \sin (q r) \mathrm{d} q}{\left|\sum_{\alpha} c_{\alpha} f_{\alpha}(q)\right|^{2}}
$$

connects the partial total correlation functions $h_{\alpha \beta}(r)$ with the total correlation function $h(r)$. Measured scattered intensities $I^{\mathrm{S}} \propto(\mathrm{d} \sigma / \mathrm{d} \Omega)(6.1)$ can be fitted to the expression

$$
S(q)=\frac{\frac{1}{N}\left(\frac{\mathrm{d} \sigma}{\mathrm{d} \Omega}\right)-\sum_{\alpha} c_{\alpha}\left|f_{\alpha}(q)\right|^{2}}{\left|\sum_{\alpha} c_{\alpha} f_{\alpha}(q)\right|^{2}}
$$

from which the real space total correlation function can be obtained by the inverse Fourier transformation of equation (6.29). 


\section{Alternative partial structure factors}

In literature alternative definitions of the partial structure factor can be found depending on the field of research. For instance, the partial structure factor after Ashcroft and Langreth [7]

$$
\begin{gathered}
S_{\alpha \beta}^{\mathrm{AL}}(q)=c_{\alpha} \delta_{\alpha \beta}+c_{\alpha} c_{\beta} \frac{4 \pi \bar{\rho}}{q} \int_{0}^{\infty}\left(g_{\alpha \beta}(r)-1\right) r \sin (q r) \mathrm{d} r \\
\left(\frac{\mathrm{d} \sigma}{\mathrm{d} \Omega}\right)=N \sum_{\alpha, \beta} f_{\alpha}(q) f_{\beta}^{*}(q) S_{\alpha \beta}^{\mathrm{AL}}(q)
\end{gathered}
$$

is mostly used in MD simulations [62], while the partial structure factor according to Faber and Ziman [45]

$$
\begin{gathered}
S_{\alpha \beta}^{\mathrm{FZ}}(q)=1+\frac{4 \pi \bar{\rho}}{q} \int_{0}^{\infty}\left(g_{\alpha \beta}(r)-1\right) r \sin (q r) \mathrm{d} r \\
\left(\frac{\mathrm{d} \sigma}{\mathrm{d} \Omega}\right)=N \sum_{\alpha} c_{\alpha}\left|f_{\alpha}(q)\right|^{2}+N \sum_{\alpha, \beta} c_{\alpha} c_{\beta} f_{\alpha}(q) f_{\beta}^{*}(q)\left[S_{\alpha \beta}^{\mathrm{FZ}}(q)-1\right]
\end{gathered}
$$

is commonly used in neutron scattering work [49]. A comprehensive overview of different structure factors can be found in [81].

\subsection{Teubner-Strey model}

In neutron and X-ray scattering of network-forming liquids and glasses it is known that molecular ordering on an intermediate range leads to a so-called prepeak or first sharp diffraction peak in the structure factor at small scattering angels [129]. This phenomenon is also observed in the structure factor of ILs, in which two or three low- $q$ peaks occur as ILs usually consist of large asymmetric ions (cf. chapter 4). Comparison of scattering data and MD simulations revealed that these low- $q$ peaks originate from different ion-ion correlations [79]. The character of the correlations can be estimated by the low- $q$ peaks' position and width. The position $q_{0}$ of the peak corresponds approximately to the spacial oscillation period $2 \pi / q_{0}$ and the width $\Delta$ to a correlation length of $2 / \Delta$ of the underlying molecular correlations [129].

A more exact description is achieved by considering general correlations in liquids obtained from thermodynamical considerations. In chapter 2 it was demonstrated that, given an intermolecular potential of finite range, all correlation functions show the same asymptotic behavior for large distances, i.e. they exhibit the same periodicity and correlation length. If the amplitudes of the correlations are large enough, also intermediate range correlations can be described approximately by equation (2.8), yielding

$$
h^{\mathrm{TS}}(r)=\frac{A}{r} \exp \left(-\frac{r}{\xi_{\mathrm{B}}}\right) \cos \left(\frac{2 \pi r}{d_{\mathrm{B}}}-\theta\right) .
$$




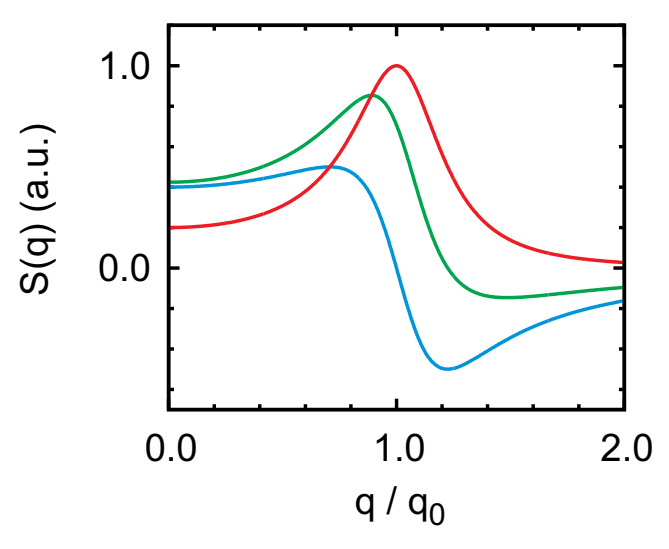

Figure 6.2: Teubner-Stey structure factor after equation (6.38b) with $\Delta=q_{0} / 2$ for different $\theta: 1 / 2 \pi$ (blue line), $5 / 4 \pi$ (green line), and $3 / 2 \pi$ (red line).

Here the correlation length $\xi_{\mathrm{B}}$ determines the decay of the correlation with periodicity $d_{\mathrm{B}}$ and phase $\theta$ relating to the test particle. From $h^{\mathrm{TS}}(r)$ an analytic form of the bulk structure factor can be determined:

$$
\begin{aligned}
S^{\mathrm{TS}}(q) & =\frac{4 \pi \bar{\rho}}{q} \int_{0}^{\infty} h(r) r \sin (q r) \mathrm{d} r \\
& =\frac{4 \pi A \bar{\rho}}{\Delta^{2}\left[1+\left(\frac{q_{0}^{2}-q^{2}}{\Delta^{2}}\right)^{2}\right]}\left(\sin \theta-\frac{q_{0}^{2}-q^{2}}{\Delta^{2}} \cos \theta\right) .
\end{aligned}
$$

The first factor in equation (6.38b) corresponds to a Lorentzian function in $q^{2}$ with peak position $q_{0}^{2}=\frac{4 \pi^{2}}{d_{\mathrm{B}}^{2}}-\frac{1}{\xi_{\mathrm{B}}^{2}}$ and width $\Delta^{2}=\frac{4 \pi}{d_{\mathrm{B}} \xi_{\mathrm{B}}}$. The oscillation period $d_{\mathrm{B}}$ and the correlation length $\xi_{\mathrm{B}}$ can be determined from $q_{0}^{2}$ and $\Delta^{2}$ :

$$
\begin{aligned}
& \xi_{\mathrm{B}}=\frac{\sqrt{2}}{\Delta^{2}}\left(q_{0}^{2}+\sqrt{\Delta^{4}+q_{0}^{4}}\right)^{1 / 2} \\
& d_{\mathrm{B}}=\sqrt{8} \pi\left(q_{0}^{2}+\sqrt{\Delta^{4}+q_{0}^{4}}\right)^{-1 / 2} .
\end{aligned}
$$

In the special case of $\theta=3 / 2 \pi,(6.38 \mathrm{~b})$ reduces to a Lorentzian function in $q^{2}$. This function was used by Teubner and Strey to describe the structure factor of density fluctuations in microemulsions $[135,153]$ and was also applied to complex IL surfactant mixtures [4].

As demonstrated in the previous section, the total structure factor $S(q)$ and the total correlation function $h(r)$ can be expressed in terms of the partial structure factors $S_{\alpha \beta}(q)$ and partial real space correlations $h_{\alpha \beta}(r)$, respectively. Equivalently $S(q)$ and $h(r)$ can be expressed by an arbitrary set of scattering density correlations $S_{i}(q)$ and real space correlations $h_{i}(r)$ :

$$
S(q)=\sum_{i} S_{i}(q)
$$




$$
h(r)=\sum_{i} A_{i} h_{i}(r)
$$

For intermediate and long distances larger than several molecular separation lengths, $S_{i}$ and $h_{i}$ can be assumed to have the form (6.37) and (6.38b), respectively. Of course, $S_{i}$ and $h_{i}$ will have contributions of different atoms, stemming from both anions and cations. However, the [FAP] $]^{-}$anion of the investigated ILs has a much higher electron density than the cation. Thus, the intermediate electron density correlations are dominated by anion-anion and anion-cation correlations leading to prominent low- $q$ peaks.

\section{Comparison to direct Fourier transformation}

In order to demonstrate that the parameterized correlations (6.37) can describe the real space correlations, a fit of the parameterized model

$$
S(q)=\sum_{i} S_{i}^{\mathrm{TS}}(q)
$$

is compared to the direct Fourier transform of high-quality XRD data from [bmpy $]^{+}[\mathrm{FPA}]^{-}$ taken at beamline 15B, ESRF Grenoble (figure 6.3a). In figure 6.3b the parameterized total correlation function

$$
h(r)=\sum_{i} h_{i}^{\mathrm{TS}}(r)
$$

is shown with $h_{i}^{\mathrm{TS}}(r)$ of form (6.37) and the direct Fourier transform (6.29) of the total structure factor $S(q)$. The Teubner-Strey fit up to $1.8 \AA^{-1}$ reproduces very well the total correlation function $h(r)$ down to $5 \AA$. This is in good agreement with results of Santos et al.[132] showing that the high- $q$ structure in $S(q)$ represents mainly intramolecular correlations and that the low- $q$ peaks contain the major part of the intermolecular correlations.

Following [132] the two low- $q$ peaks can be assigned to different ion-ion correlations. The first very sharp peak originates form anion-anion correlations and dominates the asymptotic behavior at long distances. The second much broader peak is assigned to anion-cation correlations. Indeed, the peak positions at $0.8 \AA^{-1}$ and $1.4 \AA^{-1}$ corresponds to length-scales of $8 \AA$ and $4.5 \AA$ reflecting the second and first neighbor distance, respectively. The third correlation function reflects the volume occupation of the test particle leading to a negative correlation at short distances. This results in an Lorentzian-like background (blue line in figure 6.3a).

More advanced analysis techniques involving MD simulations show that the true ion-ion correlation function exhibit more complex partial structure factors $S_{\alpha \beta}$ involving e.g. an $S_{\mathrm{ac}}$ antipeak [78], which cannot be catched by the presented model. Nevertheless, it gives the correct asymptotic behavior for intermediate and long-range correlations, at least for the studied highly correlated IL. 

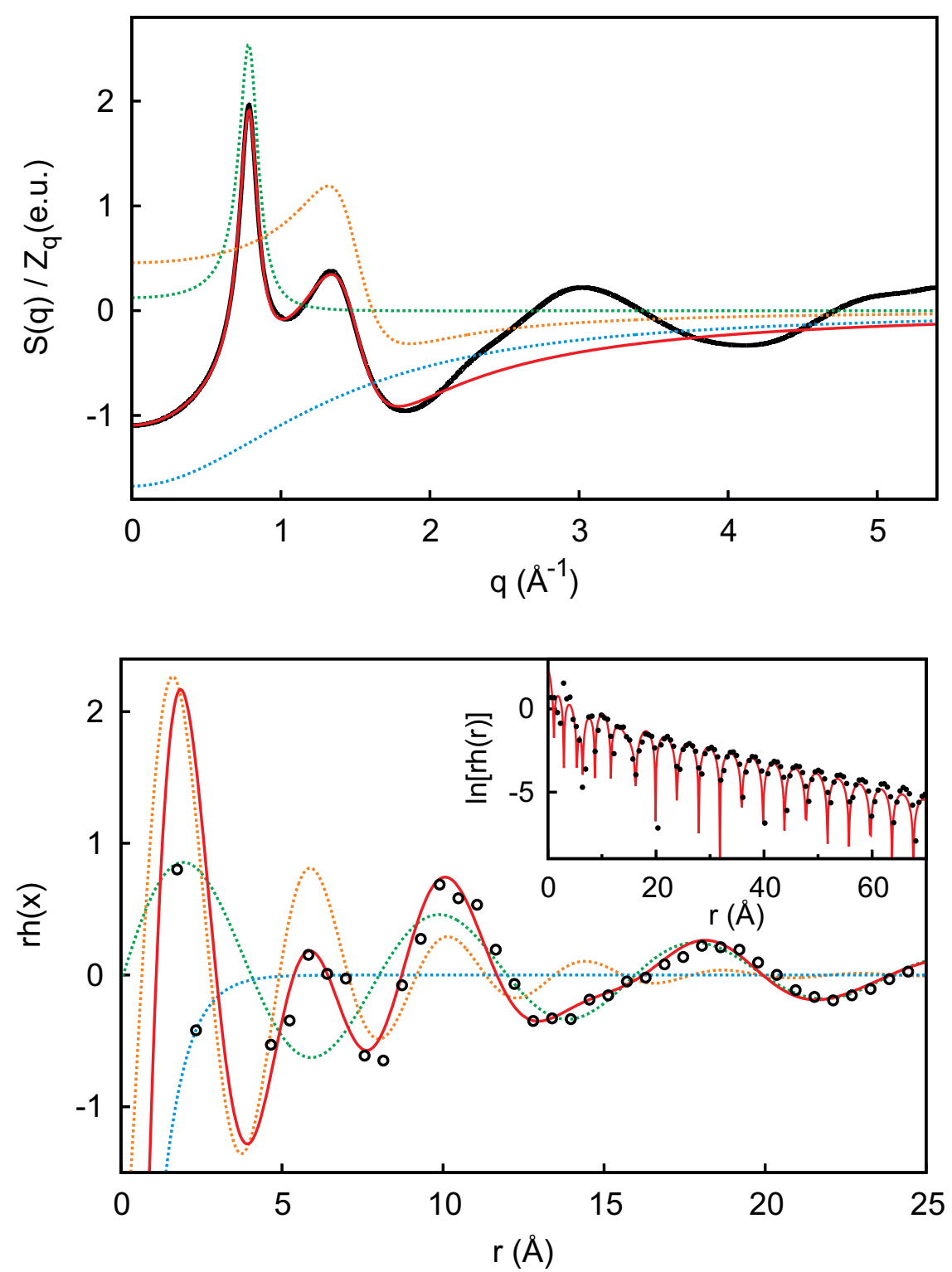

Figure 6.3: (a) Measured total structure factor $S(q)$ of $[\mathrm{bmpy}]^{+}[\mathrm{FPA}]^{-}$at $8^{\circ} \mathrm{C}$ (black symbols) with fit to Teubner-Strey model (6.43) (red line). (b) Total correlation functions determined from the Teubner-Strey model (red line) and direct Fourier transformation (symbols). The inset shows the long-range asymptotic behavior of $r h(r)$ in a logarithmic plot. The different contributions $S_{i}^{\mathrm{TS}}(q)$ and $r h_{i}^{\mathrm{TS}}(q)$ are in the same colors for both $S(q)$ and $r h(r)$ (green, blue and yellow dotted lines). 


\subsection{X-ray reflectivity}

A special case of X-ray diffraction is the specular reflection of an X-ray beam from a planar interface. This problem can be conveniently described in terms of optical refraction without necessarily applying the kinematic approximation. The discussion in this section is based on the work of M. Mezger [105]. A more comprehensive examination of the topic can be found in [159].

Optical refraction is an analogue description to the scattering formalism covered in the previous section. In this picture an electromagnetic plane wave traveling in $z$ direction through a linear medium is described by its electric field $E(z)=E_{0} \mathrm{e}^{i n(z) k_{\mathrm{z}} z}$ with the refractive index $n(z)$ of the medium. The refractive index reflects the interaction of the electromagnetic wave with the medium in which electronic transitions of the constituent atoms and molecules give rise to resonant behavior at certain frequencies. Below the resonance frequencies, i.e. in the visible part of the electromagnetic spectrum, $n$ has a value of 1.2 to 2 for most transparent materials. The increase of $n$ with increasing frequency in this range is known as normal dispersion. For X-rays, i.e. above most of the resonant frequencies, $n$ is less than unity and the deviation from the vacuum refractive index of 1 is small:

$$
n=1-\delta+i \beta
$$

From the optical theorem the deviations read

$$
\begin{gathered}
\delta=\frac{\lambda^{2}}{2 \pi} r_{\mathrm{e}} \rho_{\mathrm{e}} \frac{f^{0}(0)+f^{\prime}(\omega)}{Z} \approx \frac{\lambda^{2}}{2 \pi} r_{\mathrm{e}} \rho_{\mathrm{e}} \\
\beta=\frac{\lambda^{2}}{2 \pi} r_{\mathrm{e}} \rho_{\mathrm{e}} \frac{f^{\prime \prime}(\omega)}{Z}=\frac{\lambda}{4 \pi} \mu_{\mathrm{abs}}
\end{gathered}
$$

where $\delta$ and $\beta$ depend on the atomic form factor (6.6) of the constituent atoms and the electron density $\rho_{\mathrm{e}}$ of the medium. The imaginary part $\beta$ is related to the absorption coefficient $\mu_{\text {abs }}$. The real part $\delta$ is related to the scattering strength of the medium. A positive $\delta$ rise to the phenomenon of total reflection of an X-ray beam impinging on a matter surface from vacuum. The critical angle of total reflection can be determined by Snell's law which relates the incident angle $\alpha_{\mathrm{i}}$ and transmitted angle $\alpha_{\mathrm{t}}$ with the refractive indices of the materials

$$
n_{1} \cos \alpha_{\mathrm{i}}=n_{2} \cos \alpha_{\mathrm{t}}
$$

For an interface between a material with $n_{2}=1-\delta+i \beta$ and vacuum with $n_{1}=1$ the X-rays are totally reflected for angles smaller than the critical angle $\alpha_{\mathrm{c}}$. By setting $\alpha_{\mathrm{t}}=0$ in Snell's law, $\alpha_{\mathrm{c}}$ yields

$$
\alpha_{\mathrm{c}} \approx \sqrt{2 \delta}
$$

The relations between the incident, reflected and transmitted amplitudes are given by the Fresnel formulae. They can be deduced from the fact that the tangential component 


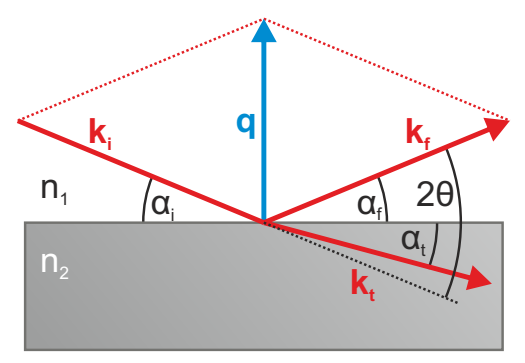

Figure 6.4: XRR geometry: The incident beam hits an interface between materials of refractive index $n_{1}$ and $n_{2}$ under the angle $\alpha_{i}$.

of the electric filed must be continuous at the interface. The reflection and transmission coefficients yield

$$
\begin{aligned}
& r=\frac{E_{\mathrm{r}}}{E_{0}}=\frac{k_{\mathrm{i}, z}-k_{\mathrm{t}, z}}{k_{\mathrm{i}, z}-k_{\mathrm{t}, z}} \\
& t=\frac{E_{\mathrm{t}}}{E_{0}}=\frac{2 k_{\mathrm{i}, z}}{k_{\mathrm{i}, z}-k_{\mathrm{t}, z}}
\end{aligned}
$$

relating the $z$-components of the wave vectors $k_{\mathrm{i}, z}$ and $k_{\mathrm{t}, z}$ to the amplitudes $E_{0}, E_{\mathrm{r}}, E_{\mathrm{t}}$. The intensity is given by the absolute square of the amplitude reflection coefficient

$$
R_{\mathrm{F}}=|r|^{2} \approx\left(\frac{\alpha_{\mathrm{c}}}{2 \alpha_{\mathrm{i}}}\right)^{4} .
$$

$R_{\mathrm{F}}$ is called Fresnel reflectivity describing the angular dependent intensity of a perfectly flat and homogeneous interface. In specular geometry, i.e. $\alpha_{\mathrm{i}}=\alpha_{\mathrm{f}}=\theta$ and $\left|\mathbf{k}_{\mathrm{i}}\right|=$ $\left|\mathbf{k}_{\mathrm{f}}\right|=k$, the wave vector transfer $\mathbf{q}=\mathbf{k}_{\mathrm{f}}-\mathbf{k}_{\mathrm{i}}$ has only a component normal to the interface:

$$
q_{z}=2 k \sin \theta=\frac{4 \pi}{\lambda} \sin \theta .
$$

\section{Arbitrary electron density profiles}

So far, only simple interfacial profiles were considered consisting of two phases of different refractive indices which are separated by a sharp interface. However, the real strength of the XRR technique lies in the determination of arbitrary refractive index profiles normal to the interface with atomic scale resolution. This task can be accomplished by dividing an arbitrary profile into a set of $N$ slices, each of refractive index $n_{j}$. The first and last slice with $j=1$ and $j=N+1$, respectively, are taken semi-infinite. The refracted amplitudes $R_{j}$ and transmitted amplitudes $T_{j}$ can be evaluated recursively at each slice via the transition coefficients $X_{j}=R_{j} / T_{j}$

$$
\begin{aligned}
X_{j} & =\varphi_{j, j} \frac{r_{j, j+1}+X_{j+1} \varphi_{j+1, j}}{1+r_{j, j+1} X_{j+1} \varphi_{j+1, j}} \\
r_{j, j+1} & =\frac{k_{j, z}-k_{j+1, z}}{k_{j, z}+k_{j+1, z}} \\
\varphi_{l, m} & =\exp \left(-2 i \mathrm{e}^{i(l+m) \pi} k_{l, z} z_{m}\right)
\end{aligned}
$$



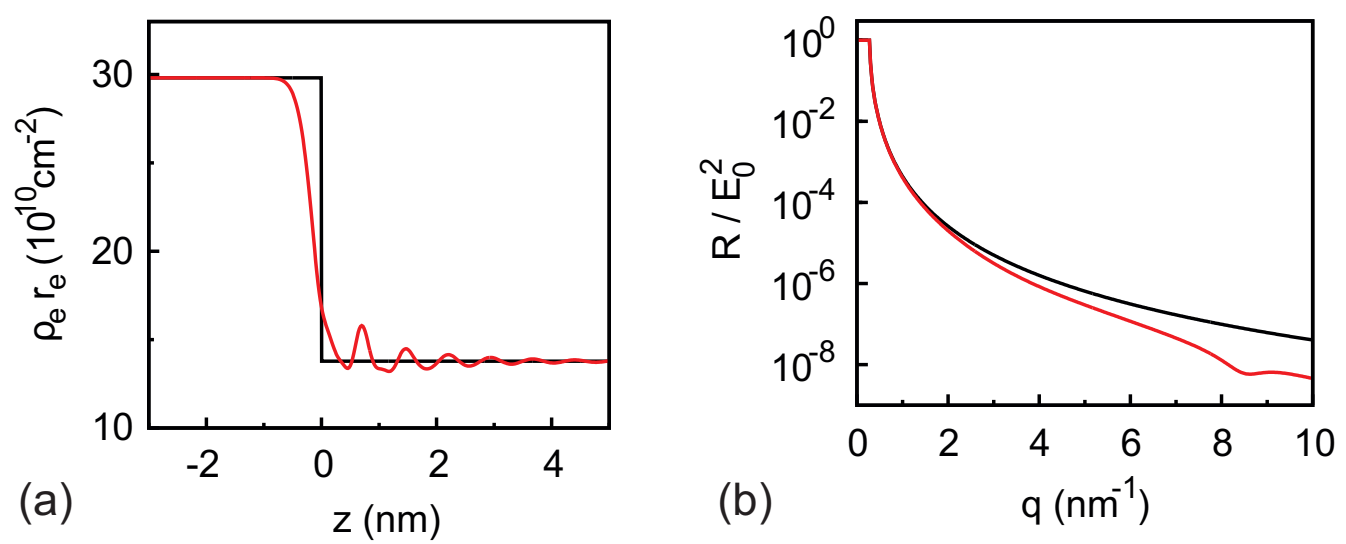

Figure 6.5: (a) Electron density of a sharp interface (black line) and of a structured interface (red line). (b) Fresnel reflectivity of a sharp interface (black line) and reflectivity intensity of the structured interface (red line) calculated via the Parratt formalism.

starting with the deepest buried slice by setting $R_{N+1}=X_{N+1}=0$. The measurable intensity, i.e. the reflectivity of all slices, is then the absolute square of the reflected amplitude of the first slice $R=\left|R_{1}\right|^{2}=\left|X_{1}\right|^{2}$. This recursive algorithm is known as the Parratt formalism [117]. In figure 6.5 the reflectivity intensity of a structured electron density profile is compared with the Fresnel reflectivity of a sharp interface.

The Parratt formalism yields an exact solution in terms of dynamical X-ray scattering. Applying the kinematic approximation (cf. section 6.1) a simple analytic relation between the measured reflectivity $R(q)$ and a continuous electron density profile $\rho_{\mathrm{e}}(z)$ can be derived, the so-called Master formula $[3,159]$

$$
\begin{aligned}
& R(q)=R_{\mathrm{F}}|F(q)|^{2} \\
& F(q)=\frac{1}{\rho_{-\infty}-\rho_{+\infty}} \int_{-\infty}^{+\infty} \mathrm{d} z \frac{\mathrm{d} \rho_{\mathrm{e}}(z)}{\mathrm{d} z} \mathrm{e}^{i q z} .
\end{aligned}
$$

At small angles around the critical angle the kinematic approximation is not valid because multiple scattering effects are dominant. However, at angles $\alpha>3 \alpha_{\mathrm{c}}$ equation (6.54) is very accurate and allows a qualitative interpretation of reflectivity data.

\subsection{Modified distorted crystal model}

In order to extract real space information from the XRR signal via the Parratt formalism, the interfacial profile has to be parameterized. An example of such a parameterized model profile is the distorted crystal model that was successfully applied in previous XRR experiments on IL/solid interfaces [108, 109]. Originally it was used to described the density oscillations at the free liquid mercury and gallium surfaces in a XRR study[102, 120]. For the present study, the interfacial profile is described by a 


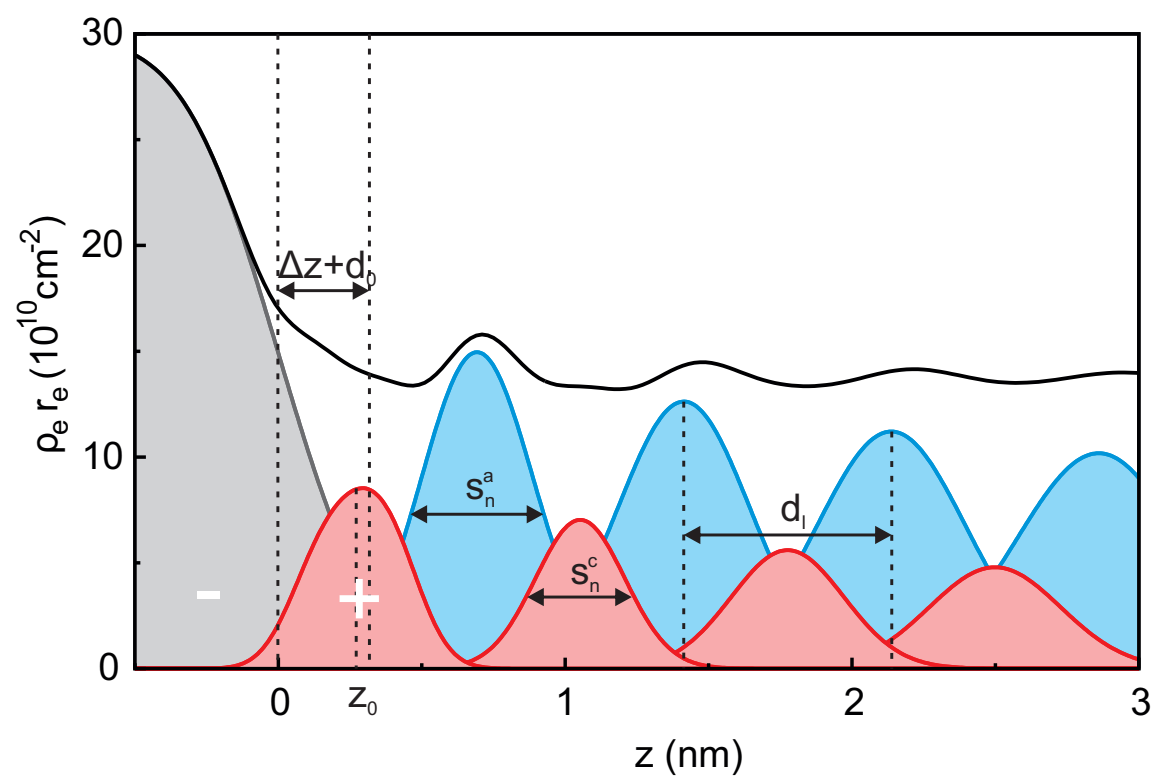

Figure 6.6: $\mathrm{MDC}$ model of the IL $[\mathrm{bmpy}]^{+}[\mathrm{FAP}]^{-}$at a boron doped diamond electrode (black line). The IL is comprised of alternating cation (red) and anion (blue) enriched layers while the elctrode is modeled by an error function (grey). The cation concentration of the layer next to the electrode compensates for the surface charge on the electrode.

Modified Distorted Crystal model (MDC), which extents the original model to a binary system comprised of anions and cations. Furthermore, it accounts for the excess surface charge in the surface adsorbed layer, induced by the applied potential.

In the MDC model the electron density profile $\rho_{\mathrm{e}}(z)$ at the liquid/solid interface is separated into solid and liquid contributions

$$
\begin{aligned}
\rho_{\mathrm{e}}(z) & =[1-\Xi(z)] \rho_{\mathrm{WE}} \\
& +\Xi(z) \sum_{n=0}^{\infty}\left[\rho_{\mathrm{c}} c_{n}^{\mathrm{c}}(z)+\rho_{\mathrm{a}} c_{n+1 / 2}^{\mathrm{a}}(z)\right] \\
\Xi(z) & =\frac{1}{2}\left[1+\operatorname{erf}\left(\frac{z}{\sqrt{2} s_{\mathrm{WE}}}\right)\right] .
\end{aligned}
$$

The solid contribution of the working electrode is modeled by an error function with bulk electron density $\rho_{\mathrm{WE}}$ and surface roughness $s_{\mathrm{WE}}$. The liquid contribution of the IL near the interface is described by a set of discrete layers of cation and anion concentration profiles $c_{n}^{\mathrm{c}}(z)$ and $c_{n}^{\mathrm{a}}(z)$ respectively. The area electron density $\rho_{\mathrm{a} / \mathrm{c}}=$ $d_{\mathrm{l}} Z_{\mathrm{a} / \mathrm{c}} \rho_{\mathrm{m}} N_{\mathrm{A}} / M_{\mathrm{a} / \mathrm{c}}$ is calculated in respect to the molar fraction of each ion species, which guarantees charge neutrality of the bulk IL. Here, $Z_{\mathrm{a} / \mathrm{c}}$ is the number of electrons per anion/cation, $\rho_{\mathrm{m}}$ the IL bulk mass density, $M_{\mathrm{a} / \mathrm{c}}$ the molecular weight of the ion species and $N_{\mathrm{A}}$ the Avogadro constant. Scaling the density with $d_{1}$ ensures the preservation of molecular volume. 
To account for adsorption and desorption of counter ions, the first ion layer, separated from the electrode by the distance $d_{0}$, is modeled by a slab profile

$$
\begin{aligned}
c_{0}^{\mathrm{c}}(z) & =E\left(z-d_{0}\right)-E\left(z-d_{0}-\Delta z\right) \\
E\left(z^{\prime}\right) & =\frac{1}{\gamma} \operatorname{erf}\left(\frac{\gamma+4 z^{\prime}}{2 \sqrt{8} w}\right) .
\end{aligned}
$$

The dimensionless parameter $\gamma$ controls the maximum possible local ion concentration $c_{\max }=2 \bar{c} / \gamma$ within the adsorbed layer with respect to the partial bulk concentration $\bar{c}$ of anions respectively cations. Thus, $\gamma$ is analog to the parameter (3.15) introduced by Kornyshev in his lattice gas model of the IL/solid interface [86]. The slab thickness $\Delta z$ is determined by the surface charge $\sigma(U)$ and was calculated numerically to obey the condition of charge neutrality

$$
\int_{-\infty}^{+\infty} \Xi(z) c_{0}^{\mathrm{c}}(z) \mathrm{d} z=\frac{1}{2}+\frac{\sigma(U)}{\sigma_{\mathrm{ML}}} .
$$

Here, the surface charge of a monolayer $\sigma_{\mathrm{ML}}$ is given by

$$
\sigma_{\mathrm{ML}}=\frac{d_{\mathrm{l}} F \rho_{\mathrm{m}}}{M}
$$

where $F$ is the Faraday constant, $\rho_{\mathrm{m}}$ the mass density, and $M$ the molar mass of the ionic liquid. The evolution of $c_{0}^{c}(z)$ with increasing $\Delta z$, i.e. increasing surface charge $\sigma(U)$, is shown in figure 6.7. The interfacial width

$$
w=\gamma\left[2 \sqrt{8} \operatorname{erf}^{-1}\left(\frac{\gamma}{\sqrt{8 \pi} s_{0}^{\mathrm{c}}}\right)\right]^{-1}
$$

is given by the constraints of equal area, equal maximum height and equal position of the turning points of the slab profile at a surface charge of $\sigma_{\mathrm{ML}} / 2$ compared to a Gaussian density distribution with width $s_{0}^{\mathrm{c}}$ (see below). The position of the first layer was defined by the center of charge, which was calculated numerically by

$$
z_{0}=\frac{\int_{-\infty}^{+\infty} \Xi(z) c_{0}^{\mathrm{c}}(z) z \mathrm{~d} z}{\int_{-\infty}^{+\infty} \Xi(z) c_{0}^{\mathrm{c}}(z) \mathrm{d} z}
$$

The subsequent layers are modeled by Gaussian distributed concentration profiles of anions and cations respectively.

$$
\begin{aligned}
& c_{n}^{\mathrm{a}}(z)=\frac{\chi_{n}^{\mathrm{a}}}{\sqrt{2 \pi} s_{n}^{\mathrm{a}}} \mathrm{e}^{-\frac{\left(n d_{1}+d_{0}+\Delta z-z\right)^{2}}{2 s_{n}^{\mathrm{a}} 2}} \\
& c_{n}^{\mathrm{c}}(z)=\frac{\chi_{n}^{\mathrm{c}}}{\sqrt{2 \pi} s_{n}^{\mathrm{c}}} \mathrm{e}^{-\frac{\left(n d_{1}+d_{0}+\Delta z-z\right)^{2}}{2 s_{n}^{\mathrm{c}} 2}}
\end{aligned}
$$




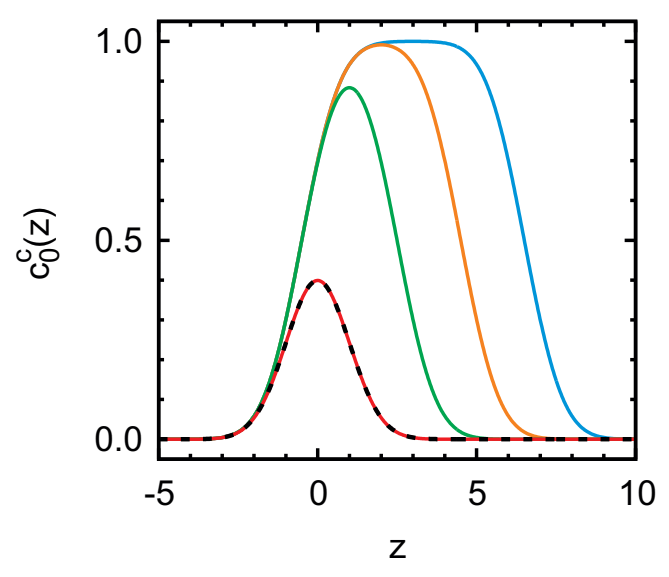

Figure 6.7: Concentration profile of the first layer with $s_{0}^{\mathrm{c}}=1$ and $\gamma=0.5$ for different values of $\Delta z: 1$ (red), 3 (green), 5 (yellow), and 7 (blue). A Gaussian with width 1 is plotted as black dashed line.

The correction factors

$$
\chi_{n}^{\mathrm{a} / \mathrm{c}}=\left[\int_{-\infty}^{+\infty} \Xi(z) c_{n}^{\mathrm{a} / \mathrm{c}}(z) d z\right]^{-1}
$$

account for the cut electron density by the substrate surface $\Xi(z)$. In equations (6.61) the bulk layers are shifted by the position $d_{0}$ and the broadening $\Delta z(U)$ of the first layer. The broadening of the layers towards the bulk IL is described by an increasing width $s_{n}^{\mathrm{a} / \mathrm{c}}=\sqrt{\left(s_{0}^{\mathrm{a} / \mathrm{c}}\right)^{2}+s(U)^{2}+n s_{\mathrm{b}}^{2}}$, where the width of the first layer $s_{0}^{\mathrm{a} / \mathrm{c}}$ is around the molecular size of the ion species. For different electrode potentials the width of the ion distributions cannot be assumed to be the same. So a potential depended broadening $s(U)$ is introduced.

\section{Extraction of asymptotic real space correlations}

Far away from the electrode, the electron density profile described by the MDC model follows the asymptotic correlation of form (2.9), in accordance with the theory of simple liquids. Thus, the interfacial correlation length $\xi_{\mathrm{I}}$ and periodicity $d_{\mathrm{I}}$ can be extracted from the electron density fluctuations $\Delta \rho_{\mathrm{e}}(z)=\rho_{\mathrm{e}}(z)-\rho_{\mathrm{IL}}$, where $\rho_{\mathrm{e}}(z)$ is given by (6.55) and $\rho_{\mathrm{IL}}$ is the mean electron density in bulk. Indeed, at distances $z>d_{1}$ the electron density profile follows the relation (figure 6.8)

$$
\Delta \rho_{\mathrm{e}}(z) \sim A_{\mathrm{I}} \exp \left(-\frac{z}{\xi_{\mathrm{I}}}\right) \sin \left(\frac{2 \pi z}{d_{\mathrm{I}}}+\theta_{\mathrm{I}}\right)
$$

where $A_{\mathrm{I}}$ is the amplitude at the electrode position $z=0$ and $\theta_{\mathrm{I}}$ is the phase of the layering in respect to the electrode. The values of $d_{\mathrm{I}}$ and $\theta_{\mathrm{I}}$, presented in chapters 9 and 10 , were retrieved by determining the roots of $\Delta \rho_{\mathrm{e}}(z)$, while $A_{\mathrm{I}}$ and $\xi_{\mathrm{I}}$ were calculated from the amplitudes at the center positions between the root. Note that in general $d_{\mathrm{I}} \neq$ $d_{1}$. 
Figure 6.8: Electron density fluctuations $\Delta \rho_{\mathrm{e}}(z)$ described by the MDC model. For clarity $\Delta \rho_{\mathrm{e}}(z)$ is multiplied with $\mathrm{e}^{z / \xi_{\mathrm{I}}} / A_{\mathrm{I}}$. The distance $z$ away from the interface is in units of the layer separation length $d_{1}$

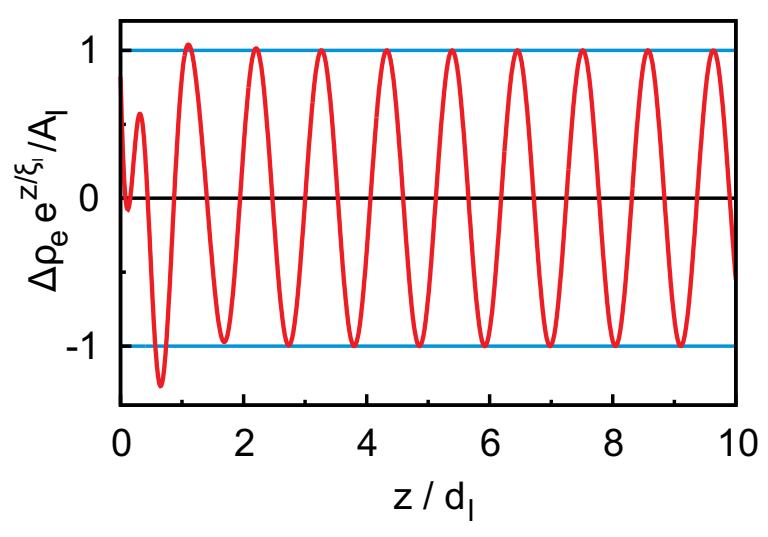

\section{Asymptotic profile in kinematic approximation}

Based on the asymptotic, oscillatory decaying electron profile (6.63), a simple model of the interfacial electron density profile can be defined [108]:

$$
\begin{aligned}
\rho_{\mathrm{e}}(z) & =[1-\Xi(z)] \rho_{\mathrm{WE}} \\
& +\Theta\left(z-d_{0}\right)\left[1+A \exp \left(-\frac{z-d_{0}}{\xi}\right) \sin \left(\frac{2 \pi\left(z-d_{0}\right)}{d}\right)\right] \rho_{\mathrm{IL}} .
\end{aligned}
$$

From this electron density profile an analytic expression of the reflectivity $R(q)$ can be calculated via the Master formula (6.54):

$$
\begin{gathered}
\frac{R}{R_{\mathrm{F}}}=\left|\left(1+\rho_{\mathrm{e}}^{\prime}\right) \mathrm{e}^{-\sigma_{\mathrm{WE}}^{2} q^{2} / 2}-\rho_{\mathrm{e}}^{\prime} \mathrm{e}^{-d_{0} q}\left[1+\mathrm{e}^{i \phi(q)} \frac{A}{2} \sqrt{q q_{0}\left[L^{+}(q)-L^{-}(q)\right]}\right]\right|^{2} \\
\phi(q)=\arctan \left(\frac{q^{2}-q_{0}^{2}-\mu^{2}}{2 \mu q}\right) \\
L^{ \pm}(q)=\left[\left(q_{0} \pm q\right)^{2}+\mu^{2}\right]^{-1}
\end{gathered}
$$

with $q_{0}=2 \pi / d$ and $\mu=\xi^{-1}$, the relative electron density of the bulk IL $\rho_{\mathrm{e}}^{\prime}=$ $\rho_{\mathrm{IL}} /\left(\rho_{\mathrm{WE}}-\rho_{\mathrm{IL}}\right)$ and of the electrode $\left(1+\rho_{\mathrm{e}}^{\prime}\right)=\rho_{\mathrm{WE}} /\left(\rho_{\mathrm{WE}}-\rho_{\mathrm{IL}}\right)$. In this model the theta function causes a non-physical electron density jump at the interface. However, the electron density several intermolecular distances away from the interface is modeled correctly. Thus, it cannot reproduce the reflectivity close to the critical angle, but it gives decent results at high $q$-values [108].

\section{Approximation of Gaussian with two error functions}

The first layer of the MDC model is described by two error functions (6.56) mimicking a Gaussian distribution. Here, the general discussion of this approximation is presented. 


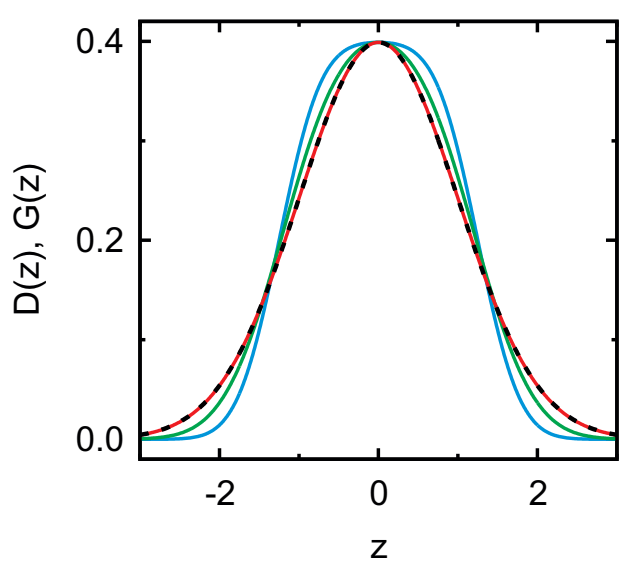

Figure 6.9: Gaussian function with width $\Delta=1$ (black dashed line) compared with double error functions $\mathrm{D}(\mathrm{z})$ with different parameters $d: 1$ (red line), 2.4 (green line), 2.5 (blue line)

A Gaussian function with width $\Delta$ is given by

$$
G(z)=\frac{1}{\sqrt{2 \pi} \Delta} \mathrm{e}^{-z^{2} / 2 \Delta^{2}}
$$

This function can be approximately described by two error functions:

$$
D(z)=\frac{1}{2 d}\left[\operatorname{erf}\left(\frac{d+2 z}{\sqrt{8} \sigma}\right)+\operatorname{erf}\left(\frac{d-2 z}{\sqrt{8} \sigma}\right)\right] .
$$

With the constraints of equal area and peak value of both functions, the following relation between $\sigma, d$ and $\Delta$ can be derived:

$$
\sigma=\frac{d}{\sqrt{8} \operatorname{erf}^{-1}\left(\frac{d}{\sqrt{2 \pi} \Delta}\right)} .
$$

For $d \rightarrow 0$ the functions (6.66) and (6.67) are identical. A set of Gaussian-like double error functions of different $d$ is compared to a similar Gaussian in figure 6.9. For further information the reader is referred to [110]. 


\section{Chapter 7}

\section{Impedance spectroscopy}

Impedance spectroscopy (IS) probes the electronic response of an electrochemical system by applying an oscillatory potential of small amplitude. In the electrochemistry, a common approach is to analyze the measured impedance spectra by means of equivalent circuits. Such an equivalent circuit can be composed of electronic components such as resistances, capacitors, and other elements $[12,21]$. Here, another approach based on the molecular picture of diffusion and relaxation is presented. This method facilitates the microscopic interpretation of experimental data and implies the same frequency dependence of the impedance as described by the equivalent circuits.

In the following a simple electrochemical system is considered. It is comprised of an electrolyte that is confined between two parallel plate electrodes (figure 7.1). In the absence of Faradaic processes, i.e. no redox reactions, no charge can pass the electrolyte/electrode interface. Such electrodes are also called blocking electrodes. When free movable charge carriers, e.g. ions, are present, the electronic response is dominated by diffusion as described in chapter 3 and not by molecular relaxation. This diffusive charging process is called electrode polarization. This process is also known in impedance spectroscopy ${ }^{1}$ of solid dielectrics, where it is recognized as an unwanted parasitic effect [88]. Although electrode polarization originates from diffusion on a macroscopic length-scale, it can be described by a Debye-like process in analogy to the description of molecular relaxation. In the following, the phenomenological concept of molecular relaxation is introduced by means of the Debye relaxation function and its more general form, the Cole-Cole relaxation function. This concept is then applied to the macroscopic effect of electrode polarization. Finally, a generalized capacitance model is presented including both molecular relaxations and electrode polarization.

\footnotetext{
${ }^{1}$ In the context of solid dielectrics such as polymers this technique is usually referred to as dielectric spectroscopy.
} 
Figure 7.1: A simple electrochemical system comprised of an electrolyte confined between two parallel plate electrodes of separation distance $L$. As soon as a potential between the electrodes is applied an electric double layer is formed at each electrode. The electronic response of the system can be modeled by a simple equivalent circuit consisting of the bulk resistance $R_{\text {eq }}$ and the capacitance $C_{\text {eq }}$. In this symmetric electrode configuration each interface contributes a capacitance of $2 C_{\text {eq }}$.

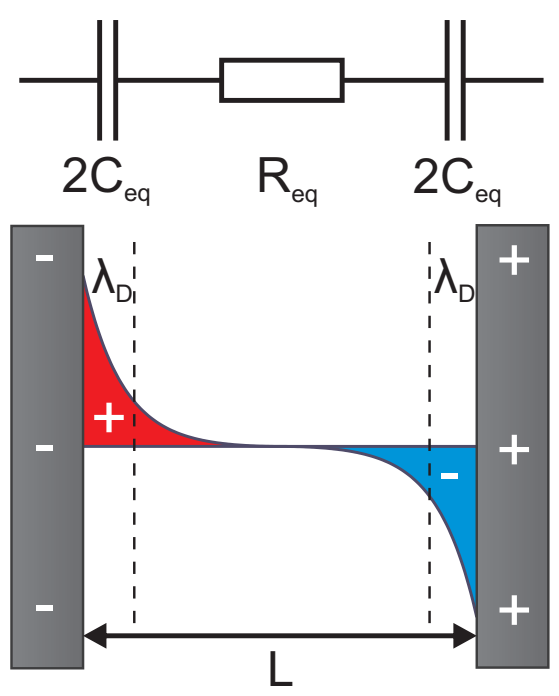

\subsection{Debye process}

The dynamic response of the polarization of matter is commonly studied by applying an oscillatory electric field $\mathbf{E}(t)=\mathbf{E}_{0} \mathrm{e}^{i \omega t}$ of amplitude $\mathbf{E}_{0}$ and frequency $\omega$. For linear, isotropic systems the polarization of the system leads to a dielectric displacement $\mathbf{D}(t)=\mathbf{D}_{0} \mathrm{e}^{i(\omega t-\delta(\omega))}$ which is out of phase with the exciting electric field by a phase $\delta(\omega)$. In analogy to the electrostatic relation $\mathbf{D}=\varepsilon \mathbf{E}$, the complex dielectric function for linear dielectrics can be introduced: ${ }^{2}$

$$
\begin{gathered}
\mathbf{D}(t)=\hat{\varepsilon}(\omega) \mathbf{E}(t) \\
\hat{\varepsilon}(\omega)=\varepsilon^{\prime}(\omega)-i \varepsilon^{\prime \prime}(\omega)=\frac{\left|\mathbf{D}_{0}\right|}{\left|\mathbf{E}_{0}\right|} \mathrm{e}^{-i \delta(\omega)}
\end{gathered}
$$

with the real part $\varepsilon^{\prime}(\omega)$ and the imaginary part $\varepsilon^{\prime \prime}(\omega)$. For linear dielectrics, where the superposition principle is valid, the frequency dependence of $\hat{\varepsilon}(\omega)$ can be treated by linear response theory. Thus, $\hat{\varepsilon}(\omega)$ can be derived by the Laplace transform $\mathcal{L}$ of the polarization pulse-response function $f_{\mathrm{P}}[19]$

$$
\begin{gathered}
\hat{\varepsilon}(\omega)=\varepsilon_{\infty}+\left(\varepsilon-\varepsilon_{\infty}\right) \mathcal{L}\left\{f_{\mathrm{P}}(t)\right\}(\omega) \\
\mathcal{L}\{y(t)\}(\omega)=\int_{0}^{\infty} \mathrm{e}^{-\omega t} y(t) \mathrm{d} t .
\end{gathered}
$$

Here we introduced the dielectric constant of induced polarization $\varepsilon_{\infty}$ stemming from intra-molecular processes, which are assumed to happen instantaneous compared to the probed frequencies. The dielectric constant of orientational polarization $\varepsilon-\varepsilon_{\infty}$ originates from the reorientation of permanent dipoles. ${ }^{3}$

\footnotetext{
${ }^{2} \mathbf{D}$ can also be expressed in terms of a polarization field: $\mathbf{D}=\mathbf{E}+\mathbf{P}$ with $\mathbf{P}=(\varepsilon-1) \mathbf{E}$

${ }^{3}$ The polarization can be separated into an induced part $\mathbf{P}_{\text {in }}(t)$ and an orientational part $\mathbf{P}_{\text {or }}(t)$ : $\mathbf{D}(t)=\mathbf{E}(t)+\mathbf{P}_{\text {in }}(t)+\mathbf{P}_{\text {or }}(t)$ with $\mathbf{P}_{\text {in }}(t)=\left(\varepsilon_{\infty}-1\right) \mathbf{E}(t)$
} 
In the Debye relaxation model the polarization follows the electric field after a steplike excitation with an exponentially damped retardation. The pulse response function of such a relaxation process is $f_{\mathrm{P}}(t)=-\frac{\mathrm{d}}{\mathrm{d} t} e^{-t / \tau}=\frac{1}{\tau} e^{-t / \tau}$ with the relaxation time constant $\tau$. The complex dielectric function (7.2a) is then given by the Debye relaxation function

$$
\hat{\varepsilon}(\omega)=\varepsilon_{\infty}+\frac{\varepsilon-\varepsilon_{\infty}}{1+i \omega \tau} .
$$

In real systems, deviations from the ideal Debye process (7.3) are observed. Thus, many extensions and corrections were proposed in literature $[19,88]$. A simple correction was proposed by K. S. Cole and R. H. Cole who introduced an additional phase parameter $\alpha$ :

$$
\hat{\varepsilon}(\omega)=\varepsilon_{\infty}+\frac{\varepsilon-\varepsilon_{\infty}}{1+i(\omega \tau)^{\alpha}}, \quad 0 \leq \alpha \leq 1 .
$$

Equation (7.4) is known as Cole-Cole relaxation function [29]. The phase parameter $\alpha$ can be associated with a distribution $G\left(\ln \tau^{\prime}\right)$ of relaxation times $\tau^{\prime}$ around $\tau$ [19]

$$
G\left(\ln \tau^{\prime}\right)=\frac{1}{2 \pi} \frac{\sin \pi \alpha}{\cosh \left[\alpha \ln \left(\tau^{\prime} / \tau\right)\right]+\cos \pi \alpha}
$$

\subsection{Electrode polarization}

The diffusion dynamics of free charge carriers in the electrolyte follow the PoissonNernst-Planck equations (cf. chapter 3). These equations can be solved for a sinusoidal electrode potential $\psi_{0}(t)=\psi_{1} \mathrm{e}^{i \omega t}$ of small amplitude $\psi_{1}[100,101]$. By assuming only small deviations $c_{1}(z) \mathrm{e}^{i \omega t}$ from the equilibrium charge carrier concentration $c_{0}(z)$, the ansatz $c(z)=c_{0}(z)+c_{1}(z) \mathrm{e}^{i \omega t}$ for the local concentration $c(z)$ can be made. Thus, the variables $t$ and $z$ in the Poisson-Nernst-Planck equations (3.26) can be separated, leading to two independent differential equations for time and space. This approximation is valid for thin diffuse layers of width $\lambda_{\mathrm{D}}$, much smaller than the electrode separation distance $L$, i.e. $\lambda_{\mathrm{D}} / L \ll 1$. In this case the diffusion from electrode to electrode on timescales $L^{2} / D$ is slow compared to the response of the electric double layer happening on timescales $\lambda_{\mathrm{D}}^{2} / D$. Thus, the charge distribution at time $t$ can be described by a quasi-static Poisson-Boltzmann distribution at electrode potential $\psi_{0}(t)$. From the frequency dependent charge carrier concentration the polarization and finally the dielectric function can be derived

$$
\begin{gathered}
\hat{\varepsilon}_{\mathrm{EP}}(\omega)=\varepsilon_{\mathrm{B}}+\frac{\Delta \varepsilon_{\mathrm{EP}}}{1+i \omega \tau_{\mathrm{EP}}} \\
\Delta \varepsilon_{\mathrm{EP}}=\left(\frac{L}{2 \lambda_{\mathrm{D}}}-1\right) \varepsilon_{\mathrm{B}} \\
\tau_{\mathrm{EP}}=\frac{\lambda_{\mathrm{D}} L}{2 D}
\end{gathered}
$$

with the bulk dielectric constant $\varepsilon_{\mathrm{B}}$. The relaxation time $\tau_{\mathrm{EP}}$ is equal to (3.27). A simplified derivation can be found in $[27,28]$ together with some amendments by Klein 
et al. [85]. Equation (7.6a) is formally equal to the Debye relaxation function (7.3), i.e. the electrolyte behaves like a macroscopic dipole.

\subsection{Capacitance model}

In impedance spectroscopy experiments the electric response of the electrochemical system stems from both molecular relaxation and electrode polarization. As the two processes exhibit similar complex dielectric functions, they can be treated by a single impedance model, which is derived in the following.

The complex impedance $\hat{Z}(\omega)=\hat{U}(\omega) / \hat{I}(\omega)$ is derived from the applied oscillatory voltage $\hat{U}(\omega)=U_{0} \mathrm{e}^{i \omega t}$ and the measured current $\hat{I}(\omega)=U_{0} \mathrm{e}^{i(\omega t-\delta(\omega))}$, which is out of phase by a frequency-dependent phase shift $\delta(\omega)$. In the absence of Faradaic currents, i.e. no charges can pass the electrolyte/electrode interface, relaxations are conveniently described in terms of the complex capacitance [88]

$$
\hat{C}(\omega)=\hat{\varepsilon}(\omega) C_{0}=\frac{1}{i \omega \hat{Z}(\omega)}
$$

For a parallel plate capacitor the dielectric function is related to the complex capacitance by its vacuum capacitance $A \cdot C_{0}$ without dielectric where $C_{0}$ is in units of capacitance per unit area $A . C_{0}$ depends on the geometry of the setup

$$
C_{0}=\frac{\varepsilon_{0}}{L}
$$

For both the molecular relaxation and electrode polarization, the complex capacitance can be described by a similar expression. By inserting the complex dielectric function (7.3) or (7.6a) in equation (7.7), respectively, the complex capacitance is obtained $^{4}$

$$
\hat{C}(\omega)=\frac{\Delta C}{1+i \omega \tau} .
$$

Here, $\Delta C$ is the capacitive strength of the process. The value of $\Delta C$ depends on the origin of the polarization. For molecular relaxation the capacitive strength yields ${ }^{5}$

$$
\Delta C_{\mathrm{R}}=\left(\varepsilon-\varepsilon_{\infty}\right) C_{0} \approx \varepsilon C_{0},
$$

while the capacitive strength of electrode polarization is ${ }^{6}$

$$
\Delta C_{\mathrm{EP}}=\Delta \varepsilon_{\mathrm{EP}} C_{0} \approx \frac{\varepsilon_{0} \varepsilon_{B}}{2 \lambda_{\mathrm{D}}}
$$

\footnotetext{
${ }^{4}$ In case of molecular relaxation (7.3) we neglected induced polarization, i.e. $\varepsilon_{\infty} \ll \varepsilon \mathrm{R}$, and in case of electrode polarization (7.6a) we assumed $\lambda_{\mathrm{D}} / L \ll 1$, which is the case for highly concentrated electrolytes.

${ }^{5}$ for $\varepsilon_{\infty} \ll \varepsilon \mathrm{R}$

${ }^{6}$ for $\lambda_{\mathrm{D}} / L \ll 1$
} 


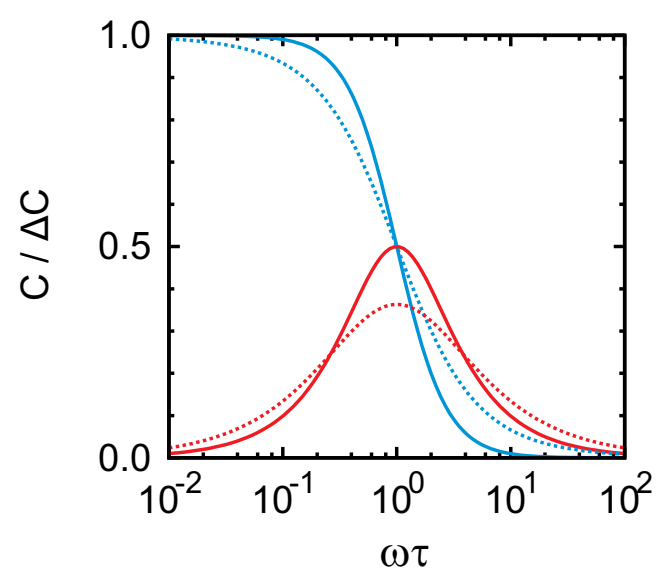

Figure 7.2: Real (blue) and imaginary (red) part of the complex capacitance described by equations (7.13a) and (7.13b), respectively. The solid lines describe the frequency dependent capacitance of a Debye process $(\alpha=1)$ or a $\mathrm{RC}$ circuit with a single relaxation time. The dotted lines describe the capacitance of a relaxation process with a relaxation time distribution $(\alpha=0.8)$.

The capacitance (7.11) is half the Debye capacitance of the diffuse double layer (3.11) at zero potential. The reduction of the Debye capacitance by a factor of 2 can be understood in the picture of an equivalent circuit in which the two electrolyte/electrode interfaces of the system are considered as two capacitors in series (figure 7.1).

In order to account for relaxation time distributions, the phase factor $\alpha$ is introduced in analogy to the Cole-Cole expression (7.4)

$$
\hat{C}(\omega)=C^{\prime}(\omega)-i C^{\prime \prime}(\omega)=\frac{\Delta C}{1+(i \omega \tau)^{\alpha}} .
$$

The complex capacitance $\hat{C}(\omega)$ can be separated into the real and imaginary part $C^{\prime}(\omega)$ and $C^{\prime \prime}(\omega)$,respectively:

$$
\begin{aligned}
C^{\prime}(\omega) & =\Delta C \frac{1+(\omega \tau)^{\alpha} \cos (\pi \alpha / 2)}{1+2(\omega \tau)^{\alpha} \cos (\pi \alpha / 2)+(\omega \tau)^{2 \alpha}} \\
C^{\prime \prime}(\omega) & =\Delta C \frac{(\omega \tau)^{\alpha} \sin (\pi \alpha / 2)}{1+2(\omega \tau)^{\alpha} \cos (\pi \alpha / 2)+(\omega \tau)^{2 \alpha}} .
\end{aligned}
$$

$C^{\prime}(\omega)$ and $C^{\prime \prime}(\omega)$ are plotted in figure 7.2 for $\alpha=1$, corresponding to an ideal Debye process with a single relaxation time, and for $\alpha=0.8$ corresponding to a process with a relaxation time distribution. Besides plotting $C^{\prime}(\omega)$ and $C^{\prime \prime}(\omega)$ versus frequency separately, the complex capacitance can be visualized in the complex plane in a single plot. Figure 7.3 shows the complex capacitance of three Cole-Cole relaxation functions with different $\alpha$ and the associated relaxation time distributions. The complex capacitance is represented by an arc in the complex plane with the maximum at frequency $\omega=1 / \tau{ }^{7}$ The capacitive strength of the process is equal to the distance between the intersections with the real axis. This means, in the relaxation model, that for low frequencies $\omega \ll 1 / \tau$ the polarization $P(t)$ follows the exciting field $E(t)$ instantaneously. This results in a maximal polarization and thus a maximal real capacitance $C^{\prime}(\omega)$. For

\footnotetext{
${ }^{7}$ note: without $2 \pi$
} 

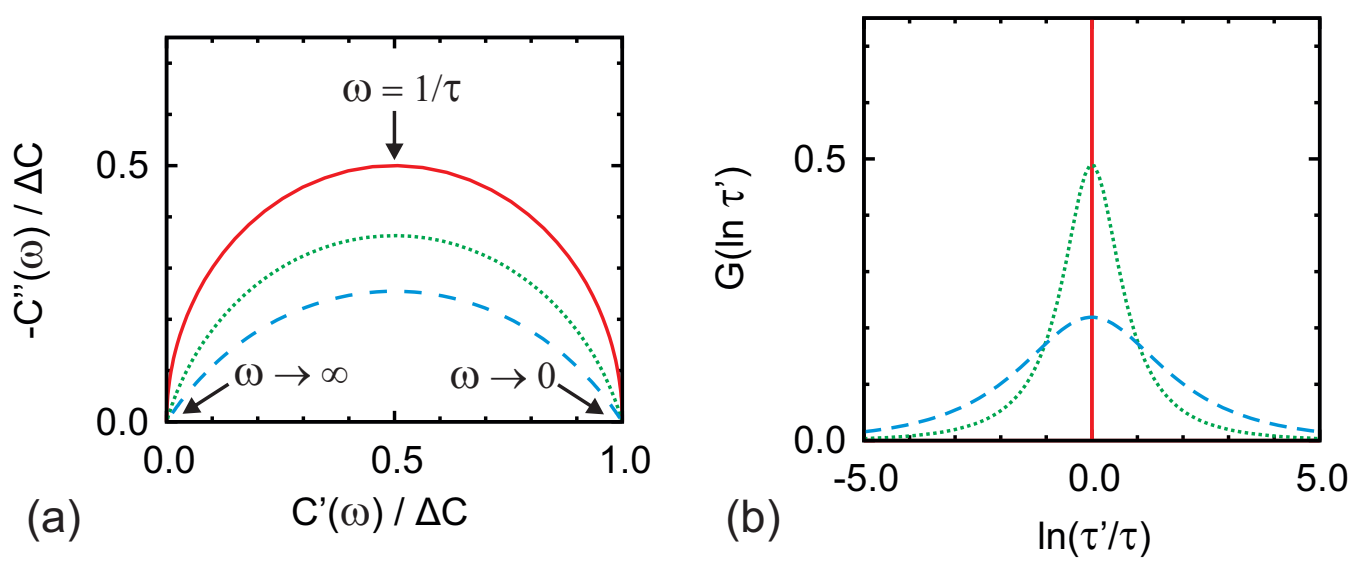

Figure 7.3: Complex capacitance (a) in the complex plane and the corresponding relaxation time distributions $G\left(\ln \tau^{\prime}\right)$ (b) for different parameters $\alpha$ : 1 (red solid line), 0.8 (green dotted line), 0.6 (blue dashed line). In figure (a) the lower arrows indicate the asymptotic behavior for $\omega \rightarrow \infty$ and $\omega \rightarrow 0$, while the upper arrow marks the frequency $\omega=1 / \tau$.

broader time distributions, i.e. $\alpha$ smaller than unity, the arcs become more and more shallow.

If more than one polarization process are present, the total complex capacitance can be written as a sum over the complex capacitances of all present processes

$$
\hat{C}(\omega)=\sum_{j=0}^{n} \frac{\Delta C_{j}}{1+\left(i \omega \tau_{j}\right)^{\alpha_{j}}} .
$$

Equation (7.14) includes both, molecular relaxation processes, and electrode polarization effects for wich the capacitive strength $\Delta C_{j}$ is given either by (7.10) or (7.11) depending on its origin. Note that for electrode polarization effects $\alpha=1$ should hold [28].

\subsection{Equivalent circuit}

The electronic response of the electrochemical system shown in figure 7.1 can also be described by a equivalent circuit consisting of a resistance in series with a capacitance. The response of this circuit to a periodic signal with angular frequency $\omega$ can be described by its impedance

$$
\hat{Z}(\omega)=R_{\mathrm{eq}}+\frac{1}{i \omega C_{\mathrm{eq}}}
$$

or its capacitance

$$
\hat{C}(\omega)=\frac{1}{i \omega \hat{Z}(\omega)}=\frac{C_{\mathrm{eq}}}{1+i \omega R_{\mathrm{eq}} C_{\mathrm{eq}}} .
$$


Equation (7.16) is formally equal to equation (7.9) with time constant $\tau=R_{\text {eq }} C_{\text {eq. }}$. For an electrode polarization process, the capacitance $C_{\mathrm{eq}}=C_{\mathrm{PB}, \mathrm{MPB}} / 2$ is given by the capacitance of the electric double layer (3.11) or (3.17). The resistance $R_{\text {eq }}=L / \sigma_{\mathrm{L}}$ is determined by the electrode separation length $L$ and the bulk conductivity

$$
\sigma_{\mathrm{L}}=\frac{N_{\mathrm{A}} e^{2}\left(c_{\mathrm{c}} D_{\mathrm{c}}+c_{\mathrm{a}} D_{\mathrm{a}}\right)}{k_{\mathrm{B}} T}
$$

The Nernst-Einstein equation (7.17) relates the the conductivity to the diffusion coefficients $D_{\mathrm{c}, \mathrm{a}}$ of cations and anions at concentrations $c_{\mathrm{c}, \mathrm{a}}$ [62]. In this way, impedance measurements can be used to determine the bulk conductivity:

$$
\sigma_{\mathrm{L}}=\frac{C_{\mathrm{eq}} L}{\tau}
$$

The description of ion diffusion in this circuit model is equivalent to the diffusion model based on the Poisson-Nernst-Planck equations (cf. section 3.3). Indeed, inserting (7.17) in (7.18) together with (3.11) yield the same relaxation time $\tau_{\mathrm{c}}$ (3.27) retrieved from the Poisson-Nernst-Planck equations. Real systems are more complicated involving electrode effects and adsorbed Stern layers, for example (cf. chapters 4 and 3). Thus, the measured capacitance $C_{\text {eq }}$ is usually much smaller than the double layer capacitance (7.11). Furthermore, equation (7.16) cannot describe deviations from ideal Debye relaxation processes with $\alpha<1$. 


\section{Chapter 8}

\section{Experimental setup and data aquisition}

In this chapter the experimental setup is described which was used to perform in situ $\mathrm{X}$-ray reflectivity experiments as well as impedance spectroscopy measurements at the IL/electrode interface. First, the sample environment and sample preparation is presented followed by the brief description of the high energy synchrotron end-station at the ESRF, where the in situ X-ray reflectivity measurements were conducted. Afterwards, an overview on the data treatment, the involved corrections and the description of the analysis of the X-ray reflectivity data is given. The identical setup was used to perform impedance spectroscopy experiments, which were conducted at the home laboratory at the MPIP. Finally, the data acquisition of bulk X-ray diffraction patterns from bulk ILs is presented.

\subsection{Electrochemical sample cell for in situ X-ray reflec- tivity}

For in situ investigation of IL/electrode interfaces a new sample cell for combined XRR and electrochemistry experiments was developed (figure 8.1). The working electrode potential is controlled by a potentiostat in three electrode configuration (PGSTAT302, Autolab, Netherlands). The working electrode (WE) consisted of a monocrystalline boron doped diamond (BDD) plate (size $4 \mathrm{~mm} \times 4 \mathrm{~mm} \times 0.3 \mathrm{~mm}$, boron concentration $10^{19} \mathrm{~cm}^{3}$, resistivity $<20 \Omega \mathrm{cm}$; Element Six, Great Britain) in (100) orientation with misscut $<3^{\circ}$. A polycrystalline highly boron doped diamond (electrochemistry grade, boron concentration $>10^{20} \mathrm{~cm}^{3}$, resistivity $<0.18 \Omega \mathrm{cm}$; Element Six, Great Britain) and a $\varnothing 2 \mathrm{~mm}$ platinum wire served as counter electrode (CE) and quasi reference electrode (RE), respectively. To get windowless access to the liquid/solid interface, the working electrode was contacted to an IL-filled PTFE trough, forming a free-standing liquid meniscus. The X-ray beam impinged on the meniscus formed between the BDD working electrode and the PTFE trough. 


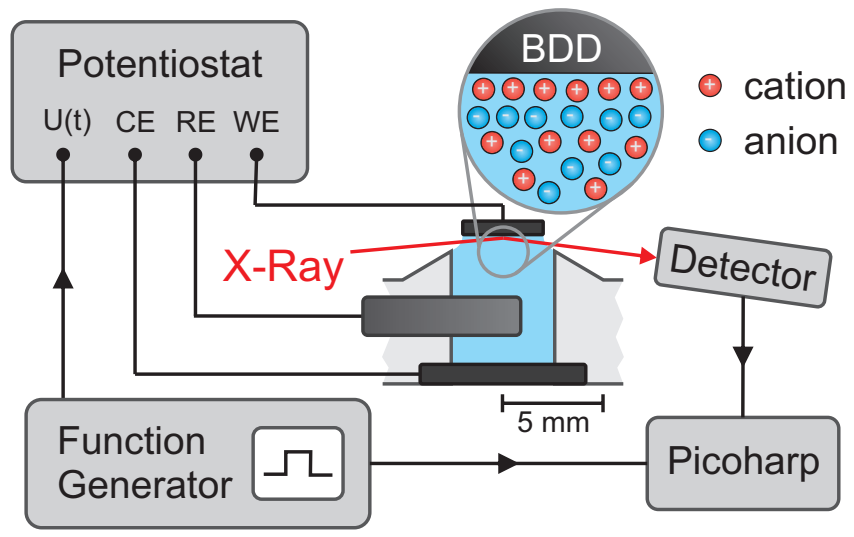

Figure 8.1: Sketch of the experimental setup for in situ IS and XRR experiments. The potential between the BDD working electrode (WE) and the platinum quasi reference electrode (RE) is controlled by a potentiostat in a three electrode configuration with a polycrystalline highly boron doped diamond counter electrode (CE). For XRR measurements with sub-millisecond time resolution, potential steps $U(t)$ were set by an external function generator. A Picoharp assigned a time stamp to each photon counting event recorded by the detector and the rising edge of the applied potential.

\section{Sample chamber}

A cut through the sample chamber is shown in figure 8.2. During the experiment the sample cell $\mathbf{A}^{1}$ was kept in the sealed sample chamber allowing a full control of the sample environment. A glass vessel $\mathbf{D}$ allowed visual inspection of the sample during alignment and measurements in vacuum or inert helium atmosphere. The pressure was controlled by a gas flow control system attached to flange $\mathbf{G}$ comprising a vacuum pump, a membrane pressure sensor and a control valve to vent with helium. The helium atmosphere prevented uncontrolled gas adsorption and reduced the background scattering in XRR experiments. To avoid temperature gradients across the sample, the temperature was controlled by two separate control loops (Lake Shore Cryotronics, US) for the working electrode and the IL containing PTFE trough. Thus, the substrate holder B of the working electrode was equipped with a heating cartridge $(40 \mathrm{~W}, 20 \mathrm{~V})$ and a PT-100 temperature sensor. The temperature of the PTFE trough $\mathbf{A}$ was controlled via a copper block C. It incorporated four heating cartridges ( $800 \mathrm{~W}$ total, $40 \mathrm{~V})$, two PT-100 sensors and a cooling apparatus, which was connected to a isopropanol filled thermostat (JULABO). The counter electrode was separated from the copper block by a single-crystalline sapphire plate (thickness $0.5 \mathrm{~mm}$ ) providing electric insulation while ensuring decent heat conductance.

Because of the small X-ray beam height of $10 \mu \mathrm{m}$ at the sample position the setup is very sensitive to drifts in vertical direction. Thus, to reduce thermally induced height drifts, the working electrode was mechanically decoupled from the copper block by an

\footnotetext{
${ }^{1}$ The notation of the parts refers to figure 8.2.
} 


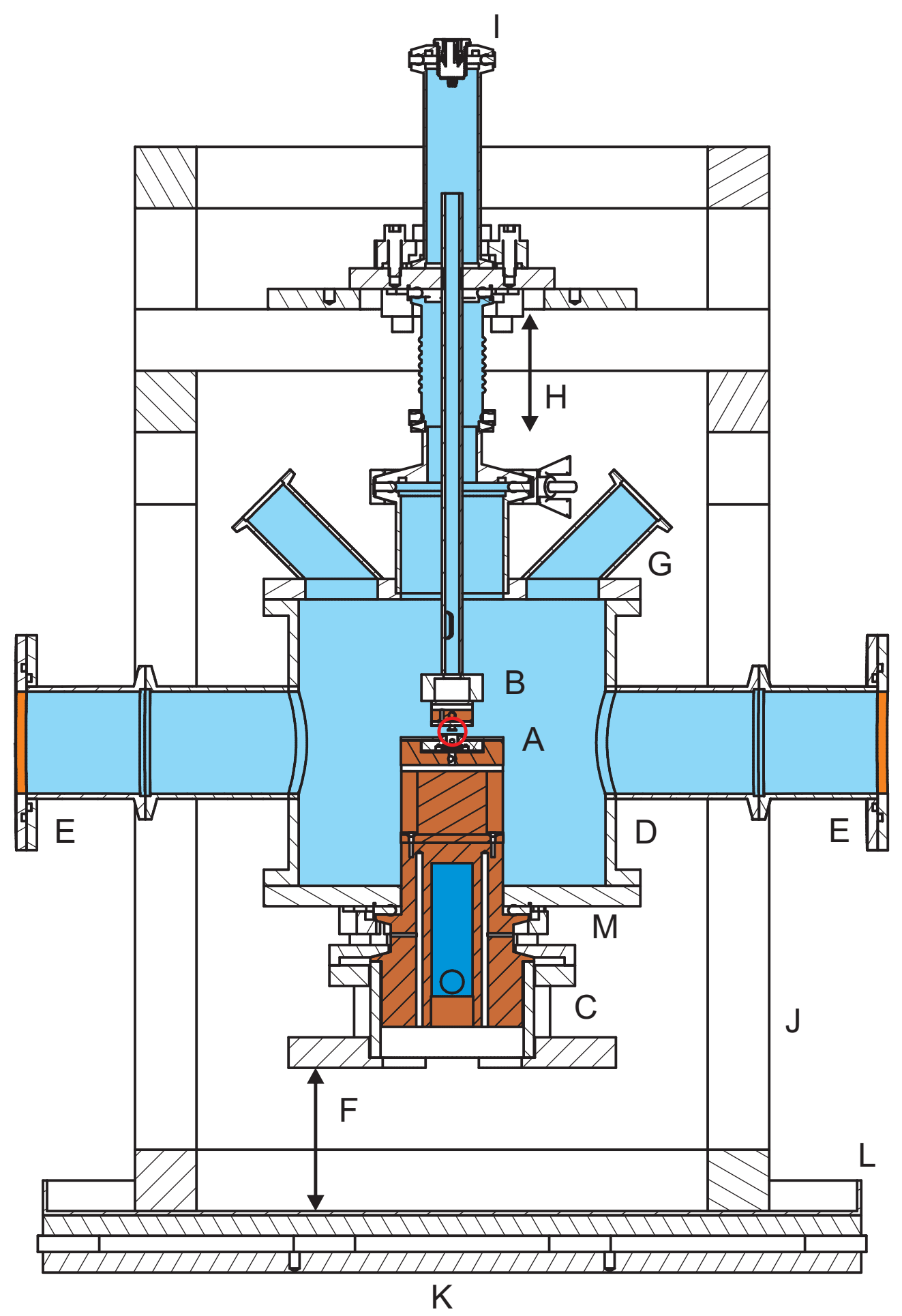

Figure 8.2: Cut through the sample chamber. The sample position is marked by a red circle. Some parts are highlighted with color: sealed volume (light blue), copper heating blocks (brown), cooling system (dark blue), Karpton windows (orange). 


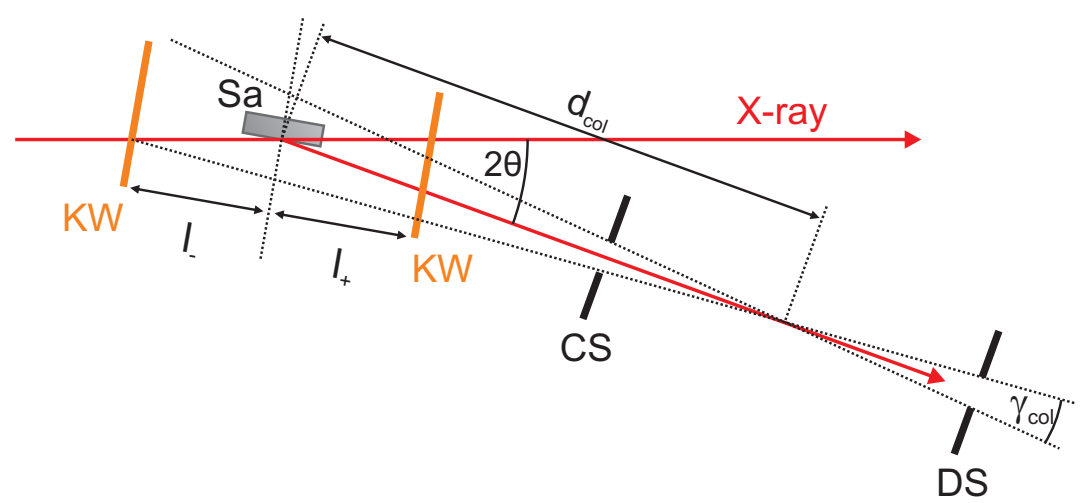

Figure 8.3: Scattering geometry of sample (Sa) at scattering angle $\theta=\theta_{\min }$. The background scattering from the entrance karpton window (KW) just passes the collimator (CS) and detector (DS) slits. The background scattering stemming from the primary $\mathrm{X}$-ray beam impinging the exit window is blocked by the slit arrangement.

aluminum profile frame $\mathbf{J}(30 \mathrm{~mm} \times 30 \mathrm{~mm}$ aluminum profile, Bosch Rexroth). The electric wires were fed into the chamber through vacuum Lemo connectors at the upper flange I for the working electrode and at the lower flange $\mathbf{M}$ for the counter and reference electrode.

\section{Entrance and exit window distance}

Considerable background scattering arises from the primary X-ray beam passing through the entrance and exit karpton windows $\mathbf{E}$. This can be blocked by the collimator slit arrangement (CS and DS in figure 8.3) at elevated angles by choosing the right window separation distance. The dependence between the sample-window distance $l$ and the minimum scattering angle $\theta_{\min }$, at which the background scattering is blocked, follows the relation

$$
l_{+/-}=d_{\mathrm{col}} \frac{\sin \left(\gamma_{\mathrm{col}} / 2\right) \cos \left(\theta_{\mathrm{min}}\right)}{\sin \left(2 \theta_{\min } \pm \gamma_{\mathrm{col}} / 2\right)}
$$

where $l_{-}$is the distance to the entrance window and $l_{+}$is the distance to the exit window. In the used setup (figures 8.3 and 8.6) the opening angle of the collimator slit arrangement is $\gamma_{\mathrm{col}}=0.19^{\circ}$ at a $1 \mathrm{~mm}$ slit opening. The sample to collimator distance (center between CS and DS in figure 8.6) is fixed to $d_{\text {col }}=900 \mathrm{~mm}$. In figure 8.4 the sample-window distances $l_{+/-}$are plotted versus the minimum scattering angle $\theta_{\min }$. For the present sample chamber both karpton windows are $210 \mathrm{~mm}$ away from the sample position. Thus, the background scattering is blocked at scattering angles larger than $0.25^{\circ}$ corresponding to a $q$-value of $3.1 \mathrm{~nm}^{-1}$ at the X-ray energy of $69.8 \mathrm{keV}$. 


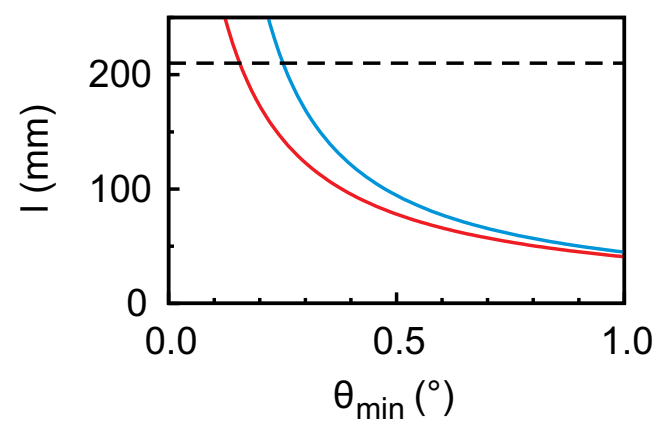

Figure 8.4: Sample-window distance $l_{-}$ for the entrance window (blue line) and $l_{+}$for the exit window (red line) vs. the minimum scattering angle $\theta_{\min }$ at which background scattering is blocked. The dashed line indicates the sample-window distance $l=210 \mathrm{~mm}$ of the sample chamber shown in figure 8.2.

\section{Sample insertion}

For synchrotron experiments a fast sample exchange is important. The decoupling of working electrode and PTFE trough allowed an effective sample insertion procedure (figure 8.2). First, the flanges $\mathbf{G}$ and $\mathbf{I}$, including the working electrode $\mathbf{B}$, were removed. After insertion of the sample and reattachment of the flanges, the IL was not in contact with the working electrode. Thus, the chamber could be evacuated and set under inert atmosphere before contacting the IL with the working electrode. The vertical translation was employed via a manual $z$-stage $\mathbf{F}$ while the position of the working electrode was fixed and decoupled by a bellow $\mathbf{H}$. This procedure allowed facile realignment in XRR experiments and prevented contamination of the sample as well as bubble formation at the electrode.

\section{Sample preparation}

Prior to the experiment all electrodes were cleaned in acetonitrile, aceton, isopropanol, and finally rinsed with ultra-pure water $(18.2 \mathrm{M} \Omega \mathrm{cm})$. The platinum wire was annealed red-hot in a butane/propane flame. The ILs were kept at $90^{\circ} \mathrm{C}$ under vacuum for over 12 hours to remove volatile solvent resistant and water. Then, the ILs were filled into the sample cell under ambient conditions, degased again for several hours and transferred to the sample chamber in a beaker filled with argon. During the alignment process of about 8 hours, prior to the XRR measurements, the sample stayed in the sample chamber under inert atmosphere. To ensure equivalent measuring conditions, the same protocol was followed for IS measurements, which were performed independently at the MPIP. Before each scan of either XRR or IS, the sample was equilibrated by applying a $50 \mathrm{~Hz}$ oscillatory potential between $-2.5 \mathrm{~V}$ and $+1.5 \mathrm{~V}$ to remove the sample history. After several minutes the amplitude was slowly decreased while the offset potential was gradually set to the final value. Following this procedure it was possible to record reproducible XRR signal from the IL/electrode interface for several potential alternations. 

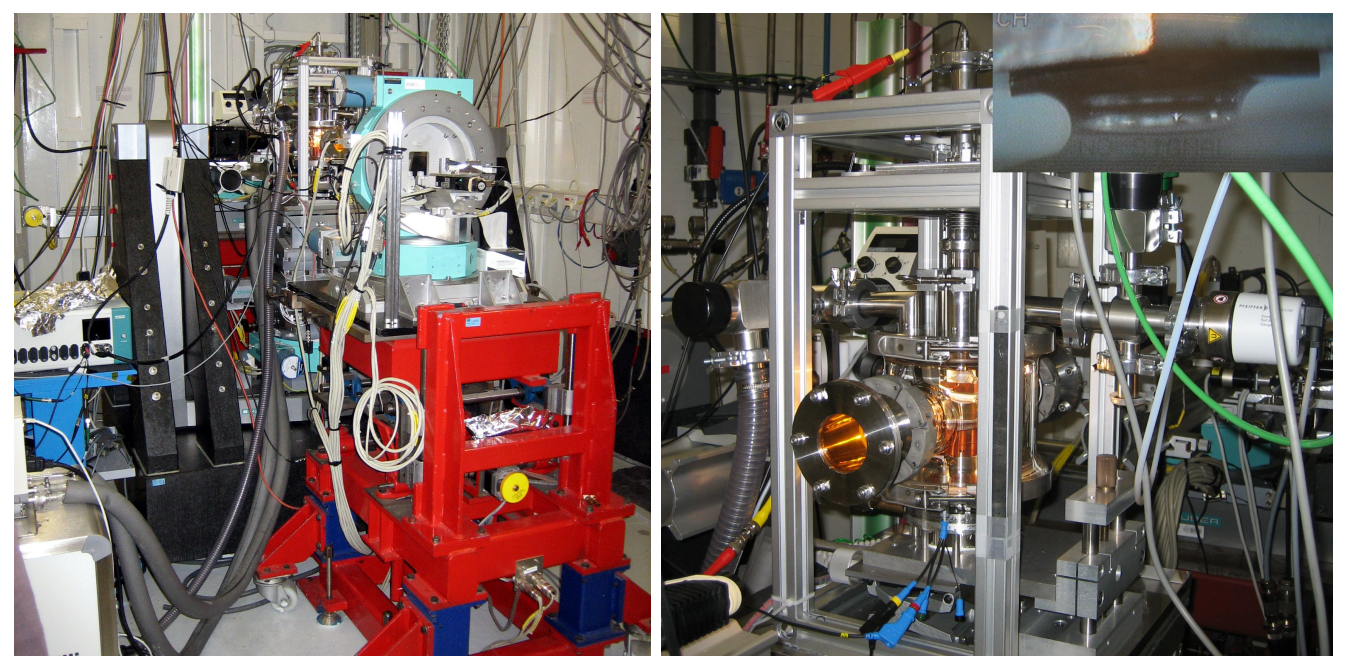

Figure 8.5: HEMD setup (left) and sample chamber (right). The inset in the right picture shows the meniscus at the BDD electrode.

\subsection{High energy $X$-ray reflectivity}

To study deeply buried structures, such as the IL/electrode interface, the use of X-ray energies larger than $25 \mathrm{keV}$ is highly desirable. In such experiments, the X-ray beam has to penetrate at least some millimeters of material. For an X-ray energy of $8 \mathrm{keV}$ provided by standard $\mathrm{X}$-ray laboratory sources using copper $\mathrm{K}_{\alpha}$ radiation, this is not possible. As the attenuation length in water is below $1 \mathrm{~mm}$. In contrast, the attenuation length in water is about $50 \mathrm{~mm}$ at an X-ray energy of $69.8 \mathrm{keV}$ used in the present study.

\section{HEMD setup}

High energy XRR experiments were performed at the high energy micro diffraction (HEMD) setup at ID15A, ESRF. This high energy scattering beamline is optimized for X-ray energies between $40 \mathrm{keV}$ and $300 \mathrm{keV}$. A layout of the the HEMD setup is shown in figure 8.6. The energy is defined by a double crystal Laue monochromator (DM) with a resolution of $\Delta E / E=2.3 \times 10^{-3}$. The beam is focused to the sample position (Sa) by a compound refractive lense (CRL) which is comprised of approx. $150-200$ aluminum lenses. The beam size at the sample position was determined to $10 \mu \mathrm{m} \times$ $50 \mu \mathrm{m}$. The incident beam intensity was monitored with the primary beam monitor diode (D1) and a secondary beam monitor diode (D2). The absorber (Ab) and the fast shutter (FS) are described in the following sections. Background scattering, stemming mainly from the absorber and the CRL, is blocked by the shielding wall (SW) and the two slit sets (S1) and (S2). In front of the scintillator counter (SC), the collimator slits (CS) and the detector slits (DS) were mounted together with a flight tube (T) on the detector arm. This arrangement blocks most of the background stemming from entrance and exit windows or air scattering. With detector diode (D3) the direct beam could be 


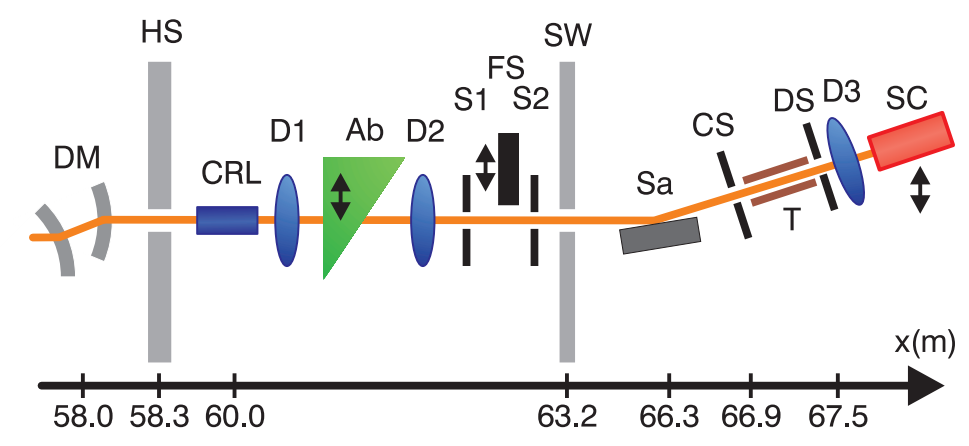

Figure 8.6: HEMD setup (taken from Mezger et al. [110]).

measured without absorber.

The sample chamber (cf. section 8.1) was mounted on a high precision diffractometer (HUBER Diffraktionstechnik GmbH \& Co. KG, Rimsting, Germany). The diffractometer was custom made for the very small scattering angles in high energy diffraction experiments. The angular accuracy is $<20 \mu \mathrm{rad}$ for the incident and $<10 \mu \mathrm{rad}$ for the exit diffractometer angel. Further, the massive design allows to install large and heavy custom sample chambers.

\section{Absorber and beam monitor}

Attenuating the incident beam with a poly(methyl methacrylate) (PMMA) absorber (triangle with thickness from 0 to $60 \mathrm{~cm}$ ) allowed to cover a dynamic range of more than eight orders of magnitude. To account for the absorbed intensity, the XRR signal was multiplied with $\mathrm{e}^{\mu_{\mathrm{PMMA}} \Delta s}$ with the absorption coefficient $\mu_{\mathrm{PMMA}}$ of PMMA and the pathway distance $\Delta s$ of the X-ray beam. To account for incident flux variations, the data was normalized by the signal of the primary beam monitor diode D1 situated between the CRL and the absorber.

\section{Fast shutter}

The fast shutter was installed to minimize radiation damage on the organic molecules of the ILs. It was synchronized with the counting command of the detector electronics. Thus, the sample was solely irradiated when the detector signal was recorded. However, the opening of the shutter was delayed by a delay time $\tau_{\text {delay }}$ compared to the counting command. Thus, the measured counting rates $N_{\text {meas }}$ had to be corrected with

$$
N_{\text {corr }}=N_{\text {meas }}\left(1+\frac{1}{t_{\text {exp }} / \tau_{\text {delay }}-1}\right) \text {. }
$$

Equation (8.2) gives the corrected count rate $N_{\text {corr }}$ for an exposure time $t_{\text {exp }}$ in which data is collected. The delay time was determined by comparing the count rates for 
Figure 8.7: Measured count rates for different exposure times (red circles) and fit to equation 8.2 (line) in order to determine the shutter delay time $\tau_{\text {delay }}$.

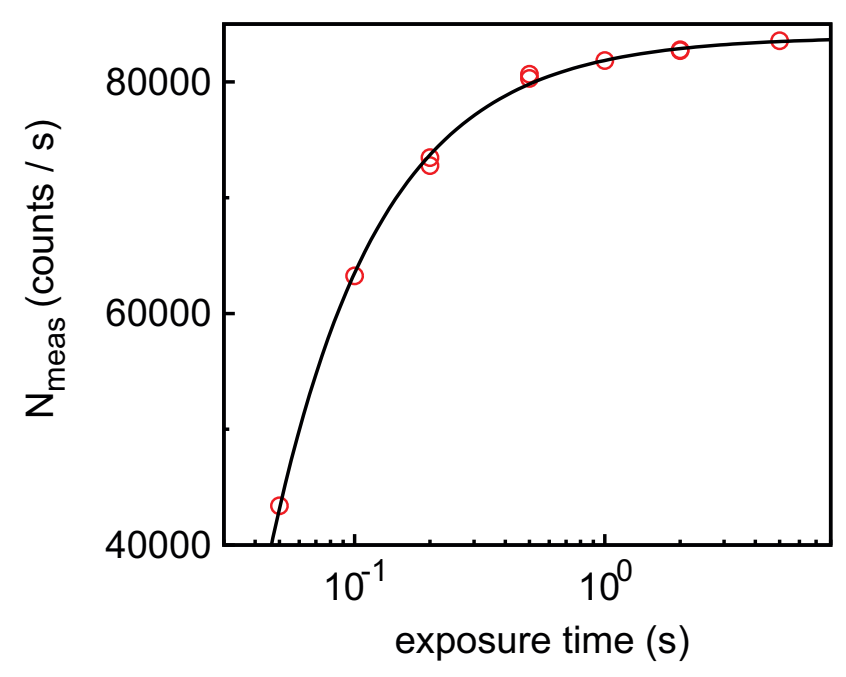

different exposure times $(0.05 \mathrm{~s}-5 \mathrm{~s})$ at constant incident flux (i.e. $N_{\text {corr }}=$ const.). The deviation from the real count rate was fitted to equation (8.2) yielding a delay time of $\tau_{\text {delay }}=24.3 \pm 0.3 \mathrm{~ms}$ (figure 8.7 ).

\section{Detector and deadtime correction}

XRR data was collected with a NaI scintillation single photon count detector (Cyberstar CBY48NA05B; Oxford Danfysik, Denmark). The incoming X-rays are converted to visible light photons that produce an electric signal via photo multiplication. The electric signal of each counting event is then post-processed by a single channel analyzer. By setting an adequate energy window, higher harmonics can be separated. Due to the thickness of the scintillation crystal of $5 \mathrm{~mm}$ the conversion efficiency is over $99 \%$ for $\mathrm{X}$-rays with energy of about $70 \mathrm{keV}$.

The measured count rates $N_{\text {meas }}$ had to be corrected for the dead time $\tau_{\text {dead }}$ of the detector. A good approximation for the dead time correction is given by

$$
N_{\text {corr }}=\frac{N_{\text {meas }}}{1-N_{\text {meas }} \tau_{\text {dead }}} .
$$

This correction is applicable for intensities well below the detector saturation (over $300000 \mathrm{cps}$ ). The dead time of the detector was determined by recording the count rate for different absorber lengths. By fitting the deviations from the expected value calculated from the absorption factor, a dead time of $0.7 \mu \mathrm{s}$ was found. In all measurements the count rates were less than $150000 \mathrm{cps}$ for which the correction factor is of about $10 \%$.

\section{Data acquisition}

Angular dependent XRR data in $\theta-2 \theta$ geometry was measured at fixed potentials. The background intensity, stemming mainly from bulk IL scattering, was collected at an off- 
set in the incidence angle of $\Delta \alpha_{i}= \pm 0.01^{\circ}$ of the the specular peak. Data collected during several subsequent scans proofed that reproducible XRR data up to a signal scattering angle $2 \theta=1.8^{\circ}$ can be attained while keeping the radiation damage of the sample at an acceptable level (figure 8.8). The dynamic range of the recorded XRR curves is about 9 orders of magnitude. Thus, the X-ray beam had to be attenuated by the absorber wedge as the the maximal countrate of the detector is approx. $300000 \mathrm{cps}$. The $2 \theta$ angle range from $0.04^{\circ}$ to $1.8^{\circ}$ was devided into 5 parts of different absorber settings. The overlay between consecutive parts was more than $0.1^{\circ}$ for the high angle scans and about half for small angel scans.

\begin{tabular}{cc} 
Table 8.1: Technical parameters of the XRR experiments. \\
\hline X-ray energy $(\mathrm{keV})$ & 69.8 \\
energy resolution $\Delta E / E(\mathrm{keV})$ & $2.3 \times 10^{-3}$ \\
primary beam intensity (photons/s) & $5 \times 10^{10}$ \\
beamsize $\left(\mu \mathrm{m}^{2}\right)$ & $10 \times 50$ \\
vertical detector slit opening $(\mathrm{mm})$ & 1.0 \\
horizontal detector slit opening $(\mathrm{mm})$ & 0.3 \\
sample-detector distance $(\mathrm{mm})$ & 1196 \\
maximum scattering vector $\left(\mathrm{nm}^{-1}\right)$ & 12 \\
sample length $(\mathrm{mm})$ & 4 \\
shutter delay time $\tau_{\text {delay }}(\mathrm{ms})$ & 24.3 \\
detector dead time $\tau_{\text {dead }}(\mu \mathrm{s})$ & 0.7 \\
\hline
\end{tabular}

Previous experiments have shown that $[\mathrm{bmpy}]^{+}[\mathrm{FAP}]^{-}$is more effected by beam damage than $[\mathrm{tba}]^{+}[\mathrm{FAP}]^{-}$, despite of the much lower temperature in the $[\mathrm{bmpy}]^{+}[\mathrm{FAP}]^{-}$ experiments. Thus, in order to reduce the influence of beam damage in $[\mathrm{bmpy}]^{+}[\mathrm{FAP}]^{-}$, the sample was translated for each data point by a distance of $40 \mu \mathrm{m}$ corresponding approximately to the horizontal beam size. For the scanned region of interest from $4 \mathrm{~nm}^{-1}$ to $11 \mathrm{~nm}^{-1}$ this amounts to a total distance of $1 \mathrm{~mm}$. The data at lower $q$-values was measured at fixed sample position where the incident X-ray intensity was considerably reduced by the absorber and beam damage is insignificant. The reproducibility of the in situ XRR signal during CV proves the stability of $[\mathrm{bmpy}]^{+}[\mathrm{FAP}]^{-}$(figure 9.9 ) and $[\mathrm{tba}]^{+}[\mathrm{FAP}]^{-}$(figure 10.5 ) over typical measurement times of over 10 minutes. A comprehensive discussion of beam damage of the ILs under investigation can be found in [134].

\section{Data treatment}

Prior to further analysis, all the presented corrections were applied to the recorded XRR intensities. These corrections include the normalization by the signal of the primary beam monitor diode, the absorption factor and the dead time correction. The shutter delay correction was only applied to the high- $q$ scans of the $[\mathrm{bmpy}]^{+}[\mathrm{FAP}]^{-} / \mathrm{BDD}$ interface, as the fast shutter was only installed in these scans. In addition, a geometrical 


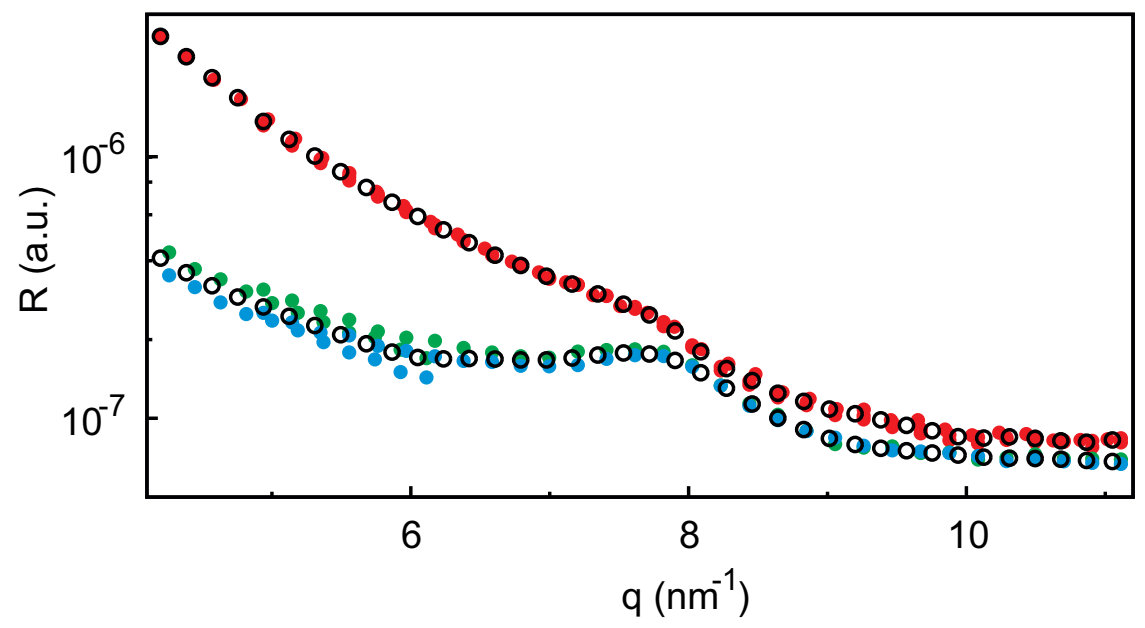

Figure 8.8: XRR data of the $[\mathrm{bmpy}]^{+}[\mathrm{FAP}]^{-} / \mathrm{BDD}$ interface at a temperature of $-12{ }^{\circ} \mathrm{C}$ and at a potential of $-2.5 \mathrm{~V}$. Full symbols represent the measured intensity from three signal scans (red), from a background scan with $\alpha_{\mathrm{i}}=\frac{2 \theta}{2}+\Delta \alpha_{\mathrm{i}}$ (blue) and a background scan with $\alpha_{\mathrm{i}}=\frac{2 \theta}{2}-\Delta \alpha_{\mathrm{i}}$ (green). The open circles represent the interpolated data.

footprint correction was applied to account for the finite beam and sample size. For a Gaussian shaped beam with width $b$ this footprint correction for a sample of length $l$ reads [159]

$$
F P=\operatorname{erf}\left(\frac{l \sin \theta}{8 \sqrt{\ln 2} b}\right)
$$

For further analysis, the specular and background signals from multiple scans were interpolated to a regular $q$-grid with an equidistant separation of $\Delta q=0.15 \mathrm{~nm}^{-1}$ (figure 8.8). As the background intensity depends on the absorber length, this procedure was applied separately for each angle range of fixed absorber setting. The interpolation function consisted of nearest-neighbor-weighted cubic polynomials. The standard deviation of the Gaussian distributed weighting factor around a grid point was empirically optimized to $0.75 \times \Delta q$ for the specular scans and $1.8 \times \Delta q$ for the background scans. Thus, the statistical noise of the background could be suppressed while all features of signal and background scans were conserved. Afterwards the scans were merged by averaging the overlaying parts beginning with the highest angle scan. In this way, the absolute intensities recorded at different electrode potentials could be compared.

\section{Error estimation}

The error of measurement is to a large extend composed of systematic errors, which are hard to quantify. The contribution of the statistical error is rather small, which can be estimated from the counting statistics of the region of interest at around $8 \mathrm{~nm}^{-1}$. At this $q$-value the signal is determined by three specular scans of 4000 counts per point taken in 
$2 \mathrm{~s}$ each and two background scans of 3000 counts per point also taken in $2 \mathrm{~s}$ each. The statistical error of the number of counting events $N$ recorded in the exposure time $t_{\text {exp }}$ can be estimated with $\sqrt{N}$ assuming underlying Poisson statistics. Then, the statistical error of the count rate $N / t_{\text {exp }}$ of both specular and background scan is about $1 \%$. The difference of both count rates gives the actual signal. Its error can be estimated to 5\% via Gaussian error propagation. This value is small compared to the signal modulation in respect to the count rate at $8 \mathrm{~nm}^{-1}$ which is $300 \%$ at $7 \mathrm{~nm}^{-1}$ and $50 \%$ at $9 \mathrm{~nm}^{-1}$.

\section{Cost function and fit algorithm}

In order to retrieve physical information from the XRR signal, the measured and preprocessed curves were fitted to a calculated reflectivity curve. The calculated curve is derived via the Parratt formalism (cf. section 6.3) from a parameterized electron density profile, which is, in this study, the modified distorted crystal model (cf. section 6.4).

The commonly used method of least squares is not suitable for the evaluation of XRR data because of its wide dynamic range. Instead of using the squared deviation of experimental values $I_{i \text {,exp }}$ and calculated values $I_{i \text {,calc }}$ as cost function, it is much more appropriate to use the logarithmic deviation $\delta_{i}=\ln I_{i, \exp }-\ln I_{i, \text { calc }}$ as cost function:

$$
\begin{aligned}
\chi^{2} & =\min _{\alpha} N^{-1} \sum_{i=1}^{N}\left[\ln \left(\alpha I_{i, \exp }\right)-\ln I_{i, \text { calc }}\right]^{2} \\
& =\min _{\alpha}\left[(\ln \alpha)^{2}+2 \ln \alpha\langle\delta\rangle+\left\langle\delta^{2}\right\rangle\right] \\
& =\left\langle\delta^{2}\right\rangle-\langle\delta\rangle^{2} .
\end{aligned}
$$

The constant $\ln \alpha=-\langle\delta\rangle$ is a normalization factor, which is adjusted after each parameter refinement. Thus, $\alpha$ is not a free fit parameter, but it makes the fit algorithm more robust against misalignment and normalization errors.

To find the best fitting physical model, the cost function $(8.5 \mathrm{c})$ has to be minimized in a multi-dimensional parameter space. However, the parameter space topology is usually not smooth but rather corrugated. Thus, deterministic fitting algorithms like the Levenberg-Marquard algorithm easily get stuck in local minima if the initial parameter guess is not sufficiently close to the global minimum. With the increased computing power stochastic approaches allow the exploration of the entire parameter space. Those algorithms less likely get trapped in local minima and the global minimum is found after a certain computation time. In this study a biased random-key genetic algorithm by Resende and Toso $[122,123]$ was implemented, which lead to satisfactory fitting results after several hours computation time on a standard PC.

\section{Time resolved XRR}

Time resolved XRR signal was recorded in the same geometry as the static XRR measurements (figure 8.1 ). The scattering angle was fixed to $2 \theta=0.7^{\circ}$ corresponding to 
a momentum transfer $q=4 \pi / \lambda \sin (\theta)=4.3 \mathrm{~nm}^{-1}$ where excellent counting statistics were obtained.

To study slow processes at a timescale of seconds, the XRR signal was recorded during cyclic voltammetry. In cyclic voltammetry the current, flowing from counter to working electrode, is recorded while the potential is swept between a maximum and minimum value with a constant scanning speed. In the experiments described in chapter 9 and 10 , the potential was scanned between $-2.5 \mathrm{~V}$ and $1.5 \mathrm{~V}$ at a scanning speed of $10 \mathrm{mV} \mathrm{s}^{-1}$ or $40 \mathrm{mV} \mathrm{s}^{-1}$.

To achieve sub-millisecond time resolution, potential steps between $-2.5 \mathrm{~V}$ and $+1.5 \mathrm{~V}$ with a frequency of $50 \mathrm{~Hz}$ were applied using a function generator. The detector signal was recorded by a Picoharp (PicoQuant, Germany) logging each single photon counting event of the detector. The electronic equipment was provided and installed by our collaborators, the group of M. M. Nielsen. Using the rising edge (rise time of $0.02 \mu \mathrm{s}$ ) of the potential steps as trigger, a sub-millisecond time resolution was achieved by rebinning of XRR data taken over several minutes at $50 \mathrm{~Hz}$. For rebinning the XRR data a MATLAB (MathWorks, MA) code was used, developed by the group of M. M. Nielsen.

\subsection{Impedance spectroscopy}

Impedance spectroscopy experiments were performed separately from the XRR experiments at the MPIP. To ensure reproducibility of the experiments, the experimental conditions and sample preparation was kept equal to the XRR experiments performed at the ESRF. Because of feasibility reasons, the sample was kept under dry nitrogen atmosphere instead of helium. Impedance spectra for electrode potentials $-2.5 \mathrm{~V} \leq U \leq+1.5 \mathrm{~V}$ were recorded in $0.25 \mathrm{~V}$ steps. For each potential the frequency was scanned over five orders of magnitude from $10 \mathrm{kHz}$ to $0.01 \mathrm{~Hz}$ with an amplitude of $10 \mathrm{mV}$. At each potential a frequency scan of about 20 minutes was started after an equilibration time of 10 minutes. To get reproducible results, impedance spectra were recorded for three full periods, each consisting of a potential scan in positive and negative direction. The spectra presented in chapter 9 were recorded in the last period.

The data was analyzed with a custom PYTHON code. First, the impedance spectra were transformed to capacitance spectra via equation (7.7). Then, the real and imaginary part of the capacitance were fitted simultaneously to equation (7.14) utilizing a generic least square fit algorithm.

\subsection{Bulk X-ray diffraction}

\section{Setup}

Bulk x-ray diffraction (XRD) data of the ILs was taken in transmission geometry using $\mathrm{Cu} \mathrm{K} \mathrm{K}_{\alpha}$ radiation $\left(\lambda_{K \alpha}=1.54 \AA\right.$, Rigaku MicroMax 007 x-ray generator, Osmic Confo- 
cal Max-Flux curved multilayer optics). The $1 \mathrm{~mm}$ thick sample was confined between two single-crystalline diamond windows to suppress background scattering. The temperature was controlled in a custom made oven. Diffraction images were recorded by a 2D image plate detector (Mar345 MarResearch) at a sample-detector distance of $342 \mathrm{~mm}$ up to a $q$-value of $2 \AA^{-1}$. The 2D-data was calibrated using a lanthanum boride standard.

\section{Data treatment}

Prior to the data reduction, a dark image was subtracted from the 2D diffraction images. An empty image was not subtracted as the scattered intensity from the diamond sample cell was negligible. Furthermore, the Compton scattering intensity was assumed to be insignificant compared to the elastically scattered intensity in the examined $q$-range. Also fluorescence and multiple scattering corrections were neglected as the sample was thin and only comprised low- $Z$ elements.

After the reduction of the $2 \mathrm{D}$ data via radial averaging, the intensity $I^{\mathrm{S}}$ scattered by the sample was retrieved from the measured $1 \mathrm{D}$ diffraction pattern $I^{\mathrm{M}}$ applying standard correction factors [145]:

$$
I^{\mathrm{S}}(\theta)=\frac{I^{\mathrm{M}}(\theta) \operatorname{geo}(\theta)}{\operatorname{Satt}(\theta) \operatorname{pol}(\theta) \text { filter }(\theta)} .
$$

Here, $g e o(\theta)$ is the geometric correction fixing the solid angle projection on the planar detector

$$
\operatorname{geo}(\theta)=\cos ^{-3}(2 \theta)
$$

and $\operatorname{pol}(\theta)$ is the polarization correction (6.3) for an unpolarized source

$$
\operatorname{pol}(\theta)=\left|\varepsilon \cdot \varepsilon^{\prime}\right|^{2}=\frac{1}{2}\left[1+\cos ^{2}(2 \theta)\right] .
$$

filter $(\theta)$ corrects for the angle dependent absorption from the diamond window situated between sample and detector:

$$
\text { filter }(\theta)=\frac{\exp \left[-\mu_{\mathrm{Di}} t_{\mathrm{fil}} / \cos (2 \theta)\right]}{\exp \left(-\mu_{\mathrm{Di}} t_{\mathrm{fil}}\right)}
$$

where $\mu_{\mathrm{Di}}=1.52 \mathrm{~mm}^{-1}$ is the absorption coefficient of the diamond window at incident $\mathrm{X}$-ray wavelength $\lambda_{K \alpha}$ and $t_{\text {fil }}=0.4 \mathrm{~mm}$ is the window thickness. The factor $\operatorname{Satt}(\theta)$ corrects for the absorbed X-rays of the sample itself

$$
\operatorname{Satt}(\theta)=\frac{\exp \left(\frac{-\mu_{\mathrm{S}} t_{\mathrm{S}}}{\sin (2 \theta+\pi / 2)}\right)\left[\exp \left(\frac{\mu_{\mathrm{S}} t_{\mathrm{S}}}{\sin (2 \theta+\pi / 2)}-\mu_{\mathrm{S}} t_{\mathrm{S}}\right)-1\right]}{\frac{\mu_{\mathrm{S}} t_{\mathrm{S}}}{\sin (2 \theta+\pi / 2)}-\mu_{\mathrm{S}} t_{\mathrm{S}}} .
$$

Here, $\mu_{\mathrm{S}}$ is the absorption coefficient of the sample material and $t_{\mathrm{S}}=1 \mathrm{~mm}$ is the thickness of the sample. 
The corrected scattered intensity $I^{\mathrm{S}}$ was normalized by comparing the diffraction pattern of [bmpy $]^{+}[\mathrm{FAP}]^{-}$at $10^{\circ} \mathrm{C}$ with high-quality synchrotron data (cf. figure 6.3). Thus, the absolute differential cross section could be retrieved. Together with the Xray form factors from [61], the liquid structure factor $S(q)$ was finally determined via equation (6.32). 


\section{Part III}

\section{RESULTS AND DISCUSSION}





\section{Chapter 9}

\section{The $[\text { bmpy }]^{+}[\text {FAP }]^{-} /$electrode interface}

In this chapter, the results of the experimental study on the $[\mathrm{bmpy}]^{+}[\mathrm{FAP}]^{-} / \mathrm{BDD}-$ electrode interface are presented. The impedance spectroscopy and XRR experiments were performed with the same experimental setup at a temperature of $-12{ }^{\circ} \mathrm{C} \mathrm{(cf.} \mathrm{sec-}$ tion 8.1). Bulk XRD patterns were recorded separately (cf. section 8.4).

First, the results of the impedance spectroscopy measurements are presented revealing three capacitive processes on different timescales. Then, the interfacial structure is examined by means of in situ XRR at different potentials and compared to the liquid bulk structure. The deployed MDC model (cf. section 6.4) comprises the surface charge determined by IS. Finally, the dynamics of the interfacial structure is compared with the relaxation times found by IS

Compared to XRR curves measured measured by other groups at IL/gold interfaces [175], the signal modulations in this study are significantly enhanced. This results from a better scattering contrast between diamond and IL than the one between Au and IL, demonstrating the high sensitivity of the used method for detecting potential-induced ion rearrangement near the electrode.

\subsection{Impedance spectroscopy results}

Impedance spectroscopy experiments were conducted at the $[\mathrm{bmpy}]^{+}[\mathrm{FAP}]^{-} / \mathrm{BDD}$ electrode interface at a temperature of $-12{ }^{\circ} \mathrm{C}$ (cf. chapter 8). The impedance spectra, recorded at electrode potentials $U$ ranging from $-2.5 \mathrm{~V}$ to $1.5 \mathrm{~V}$, reveal two distinct capacitive processes on different timescales $\tau_{1,2}(U)$ and with different relaxation strengths $\Delta C_{1,2}(U)$. Each process is represented by an arc in the complex capacitance plane (figure 9.1). Thus, the total differential capacitance $C(U)=\Delta C_{1}(U)+\Delta C_{2}(U)$ is comprised of the contributions $\Delta C_{1,2}(U)$ of the two processes. At low frequencies, the onset of a third slow process is observed.

In order to extract physical quantities, the impedance spectra were fitted to the capacitance model (7.14) consisting of three Cole-Cole relaxation functions. The resulting fit 


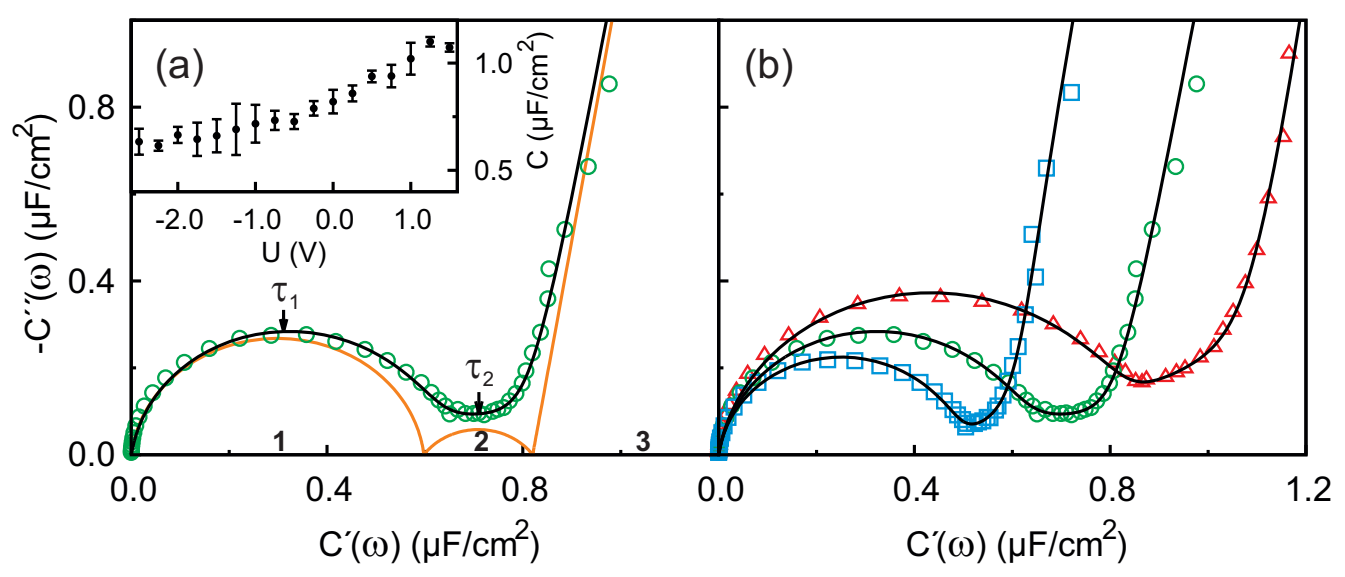

Figure 9.1: (a) Complex capacitance data at $0 \mathrm{~V}$ (green circles) with fit to equation (7.14) (black line). The yellow lines indicate the contributions of the three individual processes (numbers). Arrows point to frequencies corresponding to the relaxation times $\tau_{1}$ and $\tau_{2}$. The inset shows the total differential capacitance $C=\Delta C_{1}+\Delta C_{2}$. (b) Complex capacitance data at $-2.5 \mathrm{~V}$ (blue squares), at $0 \mathrm{~V}$ (green circles), and at $1.5 \mathrm{~V}$ (red triangles) with fits to equation (7.14) (black lines).

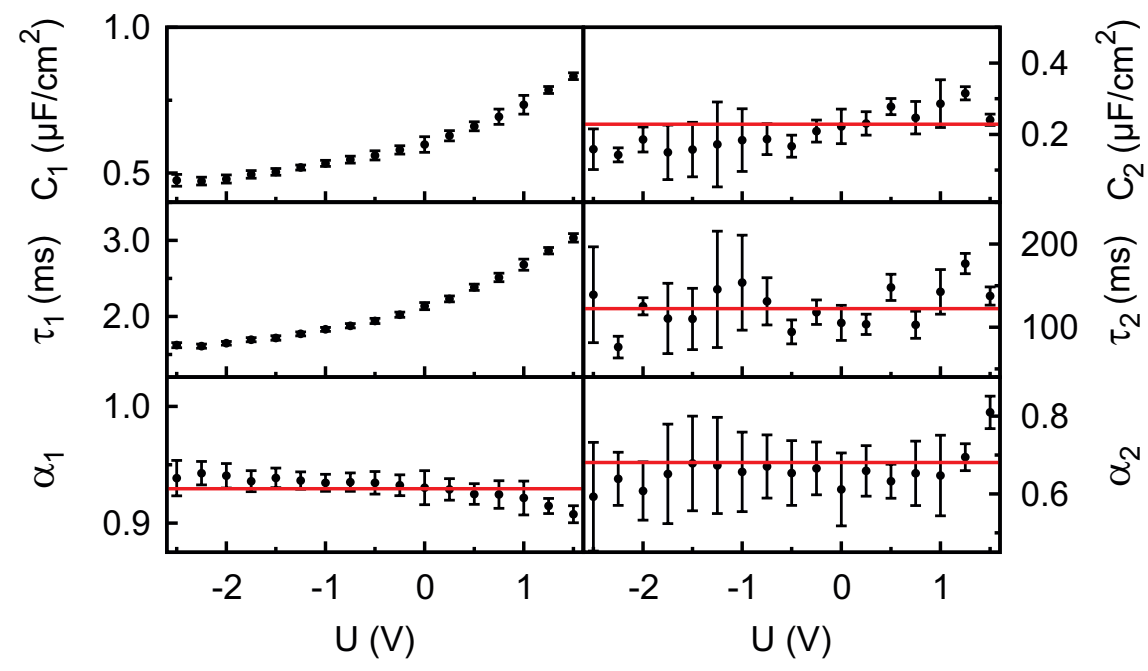

Figure 9.2: Resulting fit parameters (dots) with errors of the first process (left column) and second process (right column). Red lines indicate the mean value of all parameter values at different potentials. 


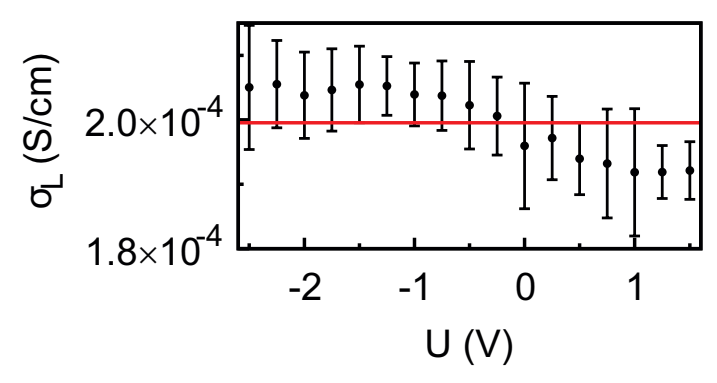

Figure 9.3: Conductivity $\sigma_{\mathrm{L}}$ (dots) with errors determined from $C_{1}$ and $\tau_{1}$ according to equation (7.18). The red line indicates the mean conductivity $\bar{\sigma}_{\mathrm{L}}=2.00 \pm$ $0.01 \times 10^{-4} \mathrm{~S} \mathrm{~cm}^{-1}$.

parameters of the first and the second process are summarized in figure 9.2. The parameters $\tau_{1,2,3}(U), \Delta C_{1,2,3}(U)$ and $\alpha_{1,2,3}(U)$ were calculated separately for each potential $U$. The red lines indicate the mean of all parameter values taken in the investigated potential range. The fit parameters of the third process are not shown as only the onset of this very slow process could be measured. Thus, large errors, compared to the associated fit parameters, hampers a reasonable physical interpretation.

The first process happens on a ms timescale and yields the largest contribution $\Delta C_{1}(U)$ to the differential capacitance. Its complex capacitance describes an almost perfect semicircle in the complex plane, i.e. its mean phase parameter $\bar{\alpha}_{1}=0.929 \pm$ 0.003 is close to unity. Such behavior is typical for a diffusion driven process (cf. chapter 7). Further, the ion conductivity ${ }^{1} \sigma_{\mathrm{L}}(U)=\Delta C_{1}(U) L / \tau_{1}(U)$ (cf. equation (7.18)) yields a potential independent value of $\bar{\sigma}_{\mathrm{L}}=2.00 \pm 0.01 \times 10^{-4} \mathrm{~S} \mathrm{~cm}^{-1}$ (figure 9.3), which is similar to the values reported in [32]. Thus, the monotonic increase of $\tau_{1}(U)$ solely originates from the increase in $\Delta C_{1}(U)$.

The second relaxation process has a small capacitive strength $\Delta C_{2}(U)$ compared to the first process. It happens on a $100 \mathrm{~ms}$ time scale. All its parameters are constant over the entire potential range, yielding a mean relaxation time $\bar{\tau}_{2}=122 \pm 7 \mathrm{~ms}$, mean capacitive strength $\overline{\Delta C}_{2}=0.23 \pm 0.01 \mu \mathrm{F} \mathrm{cm}{ }^{-2}$ and a mean phase parameter $\bar{\alpha}_{2}=$ $0.68 \pm 0.02$. The deviation of $\bar{\alpha}_{2}$ from unity is most noticeable, indicating a broad relaxation time distribution (cf. figure 7.3).

Finally, the surface charge difference $\Delta \sigma(U)$ on the electrode can be calculated by numerical integration of the total differential capacitance $C(U)=\Delta C_{1}(U)+\Delta C_{2}(U)$ :

$$
\Delta \sigma(U)=\int_{U_{0}}^{U} C\left(U^{\prime}\right) \mathrm{d} U^{\prime}
$$

The surface charge differences relative to $U_{0}=0 \mathrm{~V}$ yield $\Delta \sigma(1.5 \mathrm{~V})=1.8 \mu \mathrm{C} \mathrm{cm}^{-2}$ and $\Delta \sigma(-2.5 \mathrm{~V})=-1.9 \mu \mathrm{C} \mathrm{cm}^{-2}$. This amounts to approx. $\pm 10 \%$ of a cation monolayer, having a surface charge of $\sigma_{\mathrm{ML}}=19 \mu \mathrm{C} \mathrm{cm}^{-2}$ calculated with equation (6.58). The reduced capacitance of the $[\mathrm{bmpy}]^{+}[\mathrm{FAP}]^{-} / \mathrm{BDD}$ interface, compared to Au electrodes [32], is a consequence of the potential drop across the semiconducting BDD electrode (cf. section 5.2).

\footnotetext{
${ }^{1} L=0.7 \mathrm{~cm}$ is the separation distance between working electrode and counter electrode.
} 


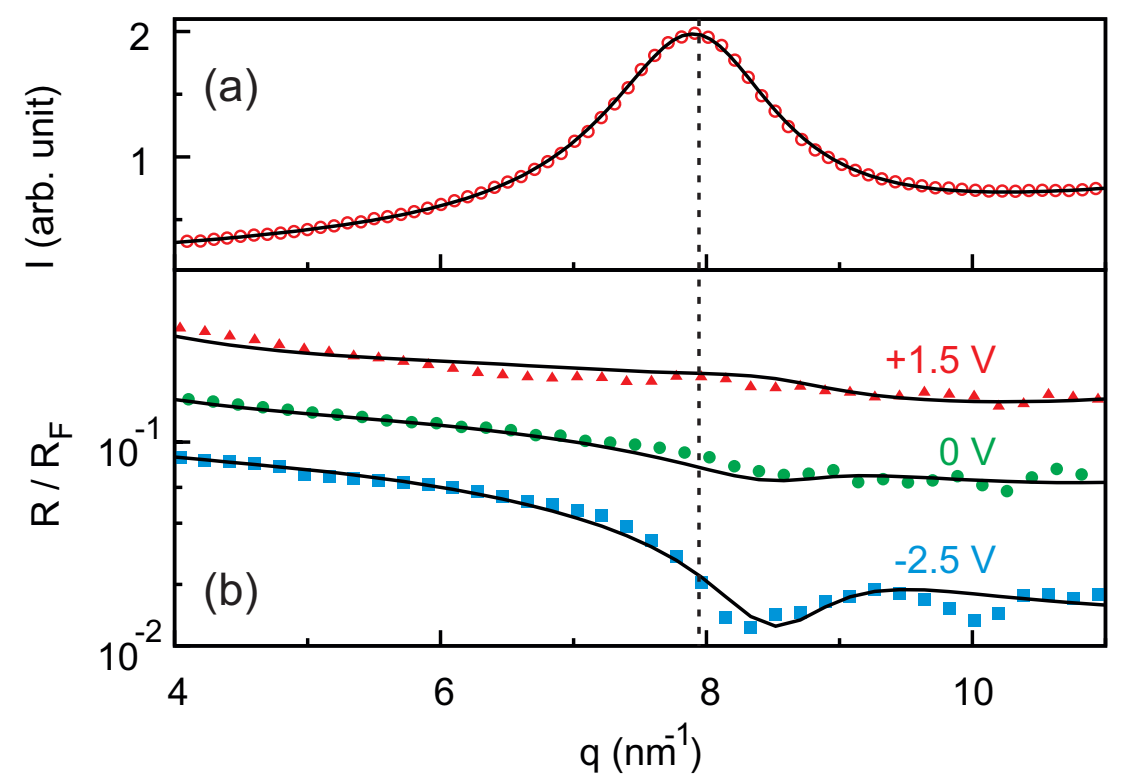

Figure 9.4: (a) Measured bulk XRD curve of $[\text { bmpy }]^{+}[\mathrm{FAP}]^{-}$(red circles) and fitted Teubner-Strey model (line). The dashed vertical line indicates $q=2 \pi d_{B}^{-1}$. (b) Measured XRR curves $R(q)$ (symbols) normalized by the Fresnel reflectivity $R_{\mathrm{F}}(q)$. Lines show simultaneous fits of all three curves to the modified distorted crystal model. The $0 \mathrm{~V}$ and $-2.5 \mathrm{~V}$ curves are shifted vertically for clarity.

\subsection{Interfacial structure}

The interface-normal ion distribution was studied by XRR measurements (cf. section 8.2). XRR curves $R(q)$ were measured at fixed potentials of $1.5 \mathrm{~V}, 0 \mathrm{~V}$ and $-2.5 \mathrm{~V}$ (figure $9.4 \mathrm{~b}$ ). The $-2.5 \mathrm{~V}$ XRR curve shows a pronounced dip at $q \approx 8 \mathrm{~nm}^{-1}$, corresponding to a distance of $2 \pi / q \approx 0.8 \mathrm{~nm}$. Its position and width are close to those of the first XRD peak of bulk samples (figure 9.4a, cf. section 8.4). Fitting the XRD peak by a Teubner-Strey form yields a periodicity of $d_{\mathrm{B}}=0.79 \mathrm{~nm}$ and a correlation length $\xi_{\mathrm{B}}=1.27 \mathrm{~nm}$ (cf. section 6.2 ). This periodicity corresponds to the average bulk separation between same-charge ions $[63,79,133]$ (cf. chapter 4). Increasing the potential from $-2.5 \mathrm{~V}$ to $1.5 \mathrm{~V}$ results in an almost structureless XRR pattern.

Table 9.1: $\quad d_{\mathrm{B}}$ and $\xi_{\mathrm{B}}$ result from fits of the Teubner-Strey form to the bulk $[\text { bmpy }]^{+}[\mathrm{FAP}]^{-}$XRD pattern. The potential independent model parameters result from the MDC model (cf. section 6.4). $\gamma$ is dimensionless, the other values are in $\mathrm{nm}$.

\begin{tabular}{ccccccccc}
\hline$d_{\mathrm{B}}$ & $\xi_{\mathrm{B}}$ & $d_{\mathrm{I}}$ & $\xi_{\mathrm{I}}$ & $d_{1}$ & $s_{0}^{\mathrm{c}}$ & $s_{0}^{\mathrm{a}}$ & $s_{\mathrm{WE}}$ & $\gamma$ \\
\hline 0.79 & 1.27 & 0.73 & 1.44 & 0.72 & 0.11 & 0.18 & 0.26 & 0.73 \\
\hline
\end{tabular}

For a quantitative interpretation, the measured XRR curves were fitted by a mod- 
Table 9.2: Potential dependent model parameters of $[\mathrm{bmpy}]^{+}[\mathrm{FAP}]^{-} /$electrode interface. The surface charge difference is in units of a full-monolayer $\sigma_{\mathrm{ML}}=19 \mu \mathrm{C} \mathrm{cm}^{-2}$. The relative values were taken from IS data, while the absolute value was fitted.

\begin{tabular}{cccc}
\hline$U(\mathrm{~V})$ & $A_{\mathrm{I}} / A_{\mathrm{I}}(U=0 \mathrm{~V})$ & $z_{0}(\mathrm{~nm})$ & $\sigma / \sigma_{\mathrm{ML}}$ \\
\hline+1.5 & 1.03 & 0.20 & $60 \%$ \\
0 & 1.00 & 0.23 & $70 \%$ \\
-2.5 & 0.84 & 0.28 & $80 \%$ \\
\hline
\end{tabular}

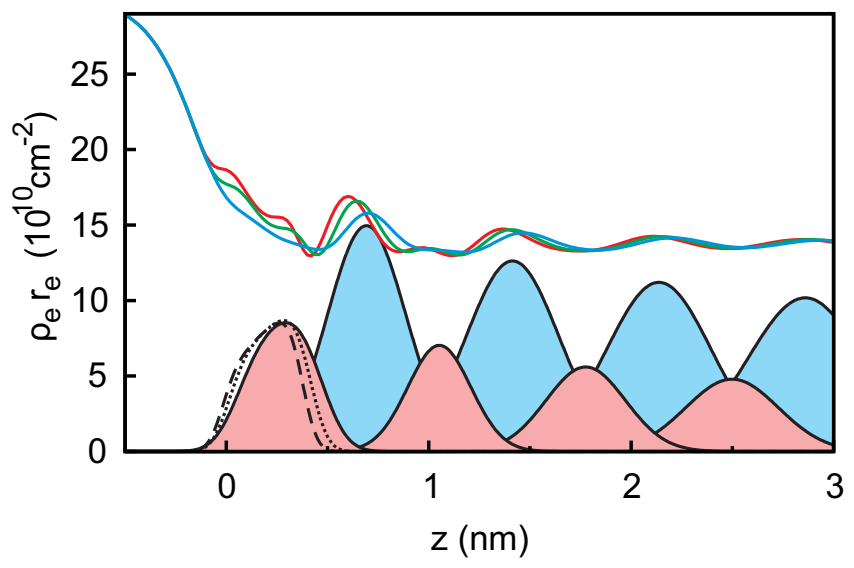

Figure 9.5: Electron density profiles extracted from the XRR curves at the potentials $1.5 \mathrm{~V}$ (red line), $0 \mathrm{~V}$ (green line) and $-2.5 \mathrm{~V}$ (blue line). The cation (light red) and anion (light blue) Gaussian distributions are plotted for a potential of $-2.5 \mathrm{~V}$. The first layer at $0 \mathrm{~V}$ (dotted black line) and at $1.5 \mathrm{~V}$ (dashed black line) are shown as well.

ified distorted crystal model (cf. section 6.4), using the Parratt formalism. The three measured XRR curves recorded at different potentials were fitted simultaneously with the same values for all bulk-related parameters, the same surface roughness $s_{\mathrm{WE}}$ of the BDD working electrode, and the fixed surface charge difference $\Delta \sigma(U)$, determined by IS. The fit results are summarized in table 9.1 showing the potential independent parameters and table 9.2 showing the potential dependent parameters. The XRR fits (lines in figure 9.4b) yield layered density profiles for both anion and cation (figure 9.5). The resultant effective interfacial layer periodicity $d_{\mathrm{I}}=0.73 \mathrm{~nm}$ and the decay length $\xi_{\mathrm{I}}=1.44 \mathrm{~nm}$ are in accordance with XRR measurements on negatively charged sapphire substrates [109] and AFM measurements that revealed a layer periodicity of $0.9 \mathrm{~nm}$ [8]. The good correspondence between $d_{\mathrm{I}}$ and $d_{\mathrm{B}}$ as well as $\xi_{\mathrm{I}}$ and $\xi_{\mathrm{B}}$ indicates that the interfacial structure is governed by the same ion-ion correlations dominating the bulk structure (cf. chapter 2).

The ion concentrations in each layer, shown in figure 9.6, were determined by integration of the interfacial model profiles, derived from the XRR fits. The interfacial profiles agree qualitatively with results from MD simulations and continuum theory at comparable surface charges (cf. figure 4.4a). The four-times-enhanced bulk correlations $\xi_{\mathrm{B}} / d_{\mathrm{B}}$ in the studied system account for the more pronounced oscillations in the relative cation/anion concentrations.

At all three potentials a cation excess was found in the substrate adsorbed layer. The overall surface charge at $1.5 \mathrm{~V}$ amounts to an equivalent of $\sim 60 \%$ of the charge in 
Figure 9.6: Anion (blue) and cation (red) charges $\sigma$ in the $n$ th IL layer, counted from the $[\text { bmpy }]^{+}[\mathrm{FAP}]^{-} / \mathrm{BDD}$ interface, at potentials $-2.5 \mathrm{~V}$ (filled), $0 \mathrm{~V}$ (hatched) and $1.5 \mathrm{~V}$ (empty). Charges are normalized to the equivalent of a full-monolayer $\sigma_{\mathrm{ML}}=19 \mu \mathrm{C} \mathrm{cm}^{-2}$.

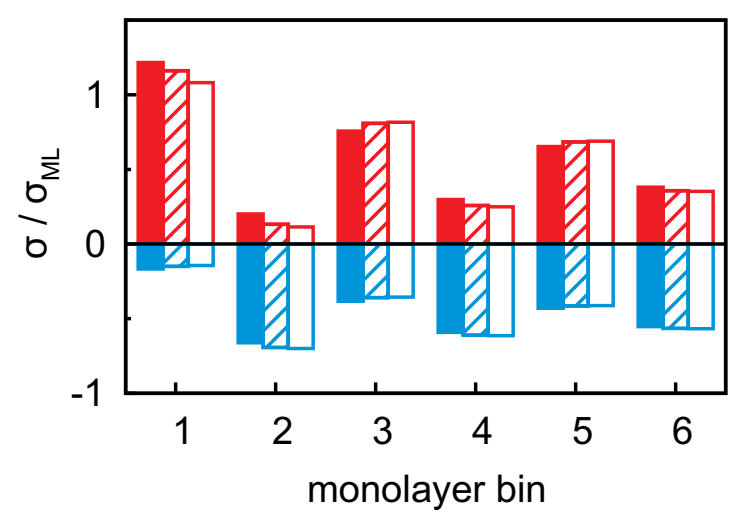

Figure 9.7: XRR patterns under variation of the first layer position. The curves are calculated with deviations of $+20 \%$ (blue line) and $-20 \%$ (red line) from the best fit value of XRR data at $-2.5 \mathrm{~V}$. The XRR curves were calculated in kinematic approximation with model (6.65).

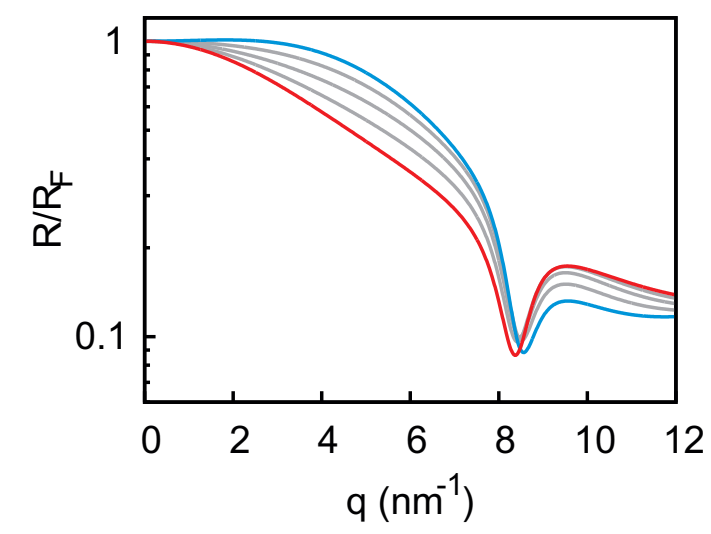

a full cation monolayer. Thus, the potential of zero charge must occur at high anodic potentials, where a maximum in $C(U)$ is predicted for symmetric ions (cf. figure 3.2). This is in agreement with the observation of a monotonically increasing $C(U)$ between a potential of $-2.5 \mathrm{~V}$ and $1.5 \mathrm{~V}$ (inset figure 9.1a).

The most prominent difference between the ion density profiles at different potentials is the shift of the center of mass $z_{0}$ of the substrate-adsorbed layer from $0.2 \mathrm{~nm}$ at $1.5 \mathrm{~V}$ to $0.23 \mathrm{~nm}$ and $0.28 \mathrm{~nm}$ at $0 \mathrm{~V}$ and $-2.5 \mathrm{~V}$, respectively (dashed, dotted and solid lines in figure 9.5). This results in a phase shift of the IL's oscillatory profile relative to the solid electrode, leading to a different interference, and hence to a different $R(q) / R_{F}(q)$ shape (figure 9.7). This explains the strong variation of the XRR signal at different potentials despite the rather small changes in the charge concentrations in all layers, shown in figure 9.6. It is remarkable, that the layering amplitude $A_{\mathrm{I}}$ is smallest at $-2.5 \mathrm{~V}$, although the XRR curve shows the strongest modulation. Shifts in the substrate-adsorbed layer position, similar to those reported here, were observed in AFM force-distance curves $[8,17,176]$. Finally, while modeling assumptions different from those employed above may slightly modify the parameter values derived from the fits, the main conclusions detailed above remain unchanged and robust. 


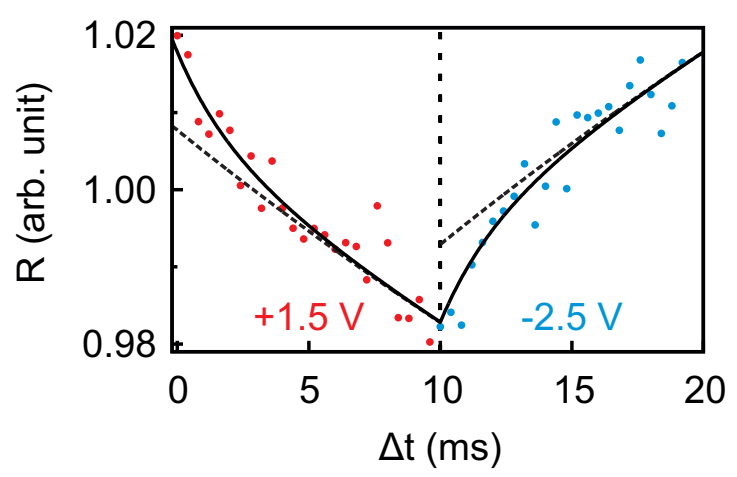

Figure 9.8: Response of the XRR signal $R$ from the $[\text { bmpy }]^{+}[\mathrm{FAP}]^{-} /$electrode interface to potential steps between $-2.5 \mathrm{~V}$ and $1.5 \mathrm{~V}$ at a frequency of $50 \mathrm{~Hz}$ (symbols). Solid lines are fits to the time dependence discussed in the text. Dashed lines are contributions from the second relaxation process.

\subsection{Dynamics}

The dynamics of ion rearrangement at the IL/electrode interface during the charging/discharging process were studied by combining the IS measurements (cf. section 9.1) with two time-resolved XRR measurements. The first, yielding sub-millisecond time resolution, recorded the evolution of the XRR signal at a fixed $q$ following an abrupt positive/negative switching of the potential $U$ (figure 9.8). The second, providing information on a longer time scale, recorded the evolution of the same fixed- $q$ XRR signal during a slow cyclic voltammetry (CV) scan of $U$ (figure 9.9). Thus, the charging/discharging process was investigated on timescales spanning several orders of magnitudes.

In the abrupt switching measurements, potential steps of $4 \mathrm{~V}$ were applied to the IL/electrode interface while recording XRR (cf. section 8.2). The resultant XRR signal shows a significant but small modulation with a relative amplitude of approximately $2 \%$. Relaxation times were determined by fitting the measured XRR by a sum of two decaying exponentials and a constant baseline

$$
R=R_{0}+R_{1} \exp \left(-\Delta t / T_{1}\right)+R_{2} \exp \left(-\Delta t / T_{2}\right)
$$

$R_{0}$ was determined from the maximal amplitude of the CV scan in figure 9.9. In table 9.3 the relaxation times $T_{1,2}$ are compared with the results of IS. The fit (figure 9.8) yields $T_{1}=1.9 \pm 2.0 \mathrm{~ms}$ in good agreement with the relaxation time $\tau_{1} \approx 2 \mathrm{~ms}$ of the first, fast process observed above by IS (cf. section 9.1). The IS experiments revealed that the phase parameter $\bar{\alpha}_{1}$ is close to unity as expected for diffusion driven electrode polarization. Further, on Au electrodes, a Vogel-Fulcher-like temperature dependence was found for this relaxation process [32]. Such dependence is characteristic of the bulk ion conductivity, suggesting that the fast relaxation process is connected to ion transport from/to the interface, limited by the ion conductivity. Indeed, its capacitive strength, $\Delta C_{1}(U)$ dominates the total capacitance found by IS (figure 9.1a) and supports this interpretation.

The magnitude $R_{2}$ of the intensity modulations associated with the second exponential (dashed lines in figure 9.8) is 14 times larger than $R_{1}$. The model fits of the XRR curves, recorded at different static potentials, show that these large modulations primarily originate from shifts of the first cation layer normal to the electrode surface 
Figure 9.9: Time resolved XRR measurements at $q=4.3 \mathrm{~nm}$ from the $[\mathrm{bmpy}]^{+}[\mathrm{FAP}]^{-} /$electrode interface. Normalized XRR intensity $R$ (symbols, solid lines are guides to the eye) and current density $I$ (dashed curve) vs. applied potential $U$ recorded during $\mathrm{CV}$ at a scanning speed of $40 \mathrm{mV} \mathrm{s}^{-1}$. Arrows show the scan directions.

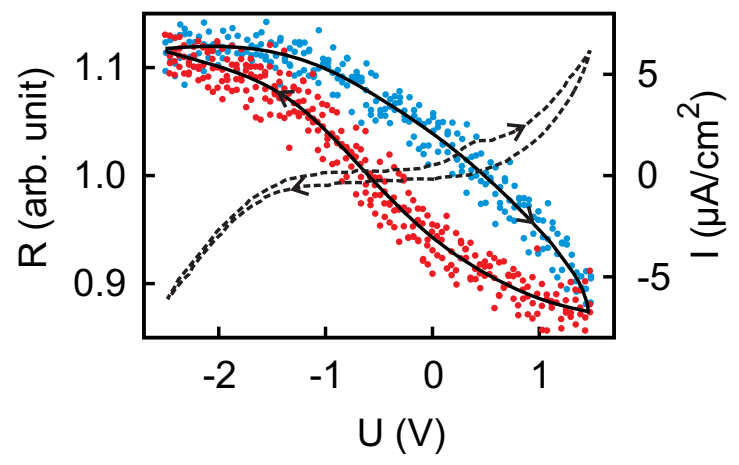

Table 9.3: Relaxation times determined by XRR and IS on the $[\mathrm{bmpy}]^{+}[\mathrm{FAP}]^{-} / \mathrm{BDD}-$ electrode system.

\begin{tabular}{rccc}
\hline XRR & \multicolumn{3}{c}{ IS } \\
\hline$T_{1}$ & $1.9 \pm 2.0 \mathrm{~ms}$ & $\tau_{1}$ & $1.6 \mathrm{~ms}-3.0 \mathrm{~ms}$ \\
$T_{2}$ & $50 \pm 16 \mathrm{~ms}$ & $\tau_{2}$ & $122 \pm 7 \mathrm{~ms}$ \\
$R_{2} / R_{1}$ & 14 & & \\
\hline
\end{tabular}

(figure 9.7). SFG spectroscopy suggests such shifts to result from potential-dependent reorientations of the asymmetric [bmpy] ${ }^{+}$substrate-adsorbed cations [11]. The value of the second relaxation time $T_{2}=50 \pm 16 \mathrm{~ms}$ is less than half of $\bar{\tau}_{2}=122 \pm 7 \mathrm{~ms}$ of the second relaxation process observed by IS. In IS, an equilibrated system is probed by small perturbations, whereas in switching measurements potential steps of $4 \mathrm{~V}$ are applied to the IL/electrode interface. This leads to a highly non-equilibrium ion configuration and a relaxation pathway with a faster time constant $T_{2}$. Apparently, this reorientation is governed by specific ion-electrode interactions and happens on much longer timescales $T_{2}$ than the ion transport. The broad relaxation time distribution obtained from IS with $\bar{\alpha}_{2}=0.68 \pm 0.02$ may reflect electrode surface inhomogeneities. The Arrhenius-like temperature dependence of $\tau_{2}$ found on gold electrodes [32] supports the assignment of this process to molecular reorientation within the first adsorbed cation layer. Finally, note that, compared to the fast ion transport process, the slower reorientation process has only a small capacitive strength $\overline{\Delta C_{2}}=0.23 \pm 0.01 \mu \mathrm{F} \mathrm{cm}^{-2}$. This may arise from the relaxation of the first cation layer's distance from the electrode surface, as well as the adsorption of additional cations on vacancies formed after reorientation.

In the low frequency regime, i.e. on the timescale above $10 \mathrm{~s}$, the IS data (figure 9.1a) indicates the onset of a third, very slow, process. This agrees with the existence of a hysteresis loop in the XRR signal recorded during CV (figure 9.9). The presence of such loop confirms the occurrence of structural rearrangements on a timescale over which a significant potential variation is affected in a CV scan, i.e. $10-100 \mathrm{~s}$. Such slow dynamics could be caused by a lateral reorganization and eventually $2 \mathrm{D}$ ordering of the 
first layer of interface-adsorbed cations, as observed in scanning tunneling microscopy [149] that also shows very slow dynamics upon potential variation [41]. 


\section{Chapter 10}

\section{The $[\text { tba }]^{+}[\mathrm{FAP}]^{-} /$electrode interface}

The second investigated IL $[\mathrm{tba}]^{+}[\mathrm{FAP}]^{-}$proofed to be much more stable under X-ray irradiation than the IL $[\mathrm{bmpy}]^{+}[\mathrm{FAP}]^{-}$. Although the experiments were conducted at a much higher temperature of $82^{\circ} \mathrm{C}$, well above the melting temperature of $[\mathrm{tba}]^{+}[\mathrm{FAP}]^{-}$, it was possible to record the XRR signal during $\mathrm{CV}$ for three entire periods at a scanning speed of $10 \mathrm{mV} \mathrm{s}^{-1}$, corresponding to over $30 \mathrm{~min}$, without any noticeable beam damage. In contrast, the IS measurements were not as reproducible as the ones reported in section 9.1. Although, also three distinct processes were observed, the related relaxation times varied considerably, yielding $\tau_{1} \approx 10^{-2}-10^{-1} \mathrm{~ms}$ for the first process and $\tau_{2} \approx 10^{1}-10^{3} \mathrm{~ms}$ for the second process. Also the capacitive strengths did not provide reliable values. Hence, the surface charge difference $\Delta \sigma(U)$ could not be determined. Taking this into consideration, only the XRR data and its analysis is discussed in the following. While the data does not provide the full picture of the $[\mathrm{tba}]^{+}[\mathrm{FAP}]^{-} /$electrode interface, it confirms the findings from the other IL/electrode system.

\subsection{Interfacial structure}

The XRR curves of the $[\mathrm{tba}]^{+}[\mathrm{FAP}]^{-} /$electrode interface were measured at fixed potentials of $1.5 \mathrm{~V}, 0 \mathrm{~V}$, and $-2.5 \mathrm{~V}$ (figure $10.1 \mathrm{~b}$ ). They show a similar dependence on the applied potential as the XRR curves recorded from the $[\mathrm{bmpy}]^{+}[\mathrm{FAP}]^{-} /$electrode interface. The dip is most pronounced at $-2.5 \mathrm{~V}$, but shifted to lower $q$-values coinciding with the first XRD peak of the bulk liquid at $q \approx 7 \mathrm{~nm}^{-1}$ (figure 10.1a). The broader $\mathrm{XRD}$ peak indicates that correlations in $[\mathrm{tba}]^{+}[\mathrm{FAP}]^{-}$are less pronounced compared to the ones observed in $[\mathrm{bmpy}]^{+}[\mathrm{FAP}]^{-}$. Indeed, the fit to a Teubner-Strey form reveals a smaller bulk correlation length $\xi_{\mathrm{B}}=0.91 \mathrm{~nm}$ at a larger periodicity $d_{\mathrm{B}}=0.91 \mathrm{~nm}$.

The XRR curves were fitted to a MDC model with similar constraints as described in section 9.2. The bulk related values and the electrode roughness $s_{\text {WE }}$ was kept equal for all potentials. The fit results of the potential independent parameters are shown in table 10.1, while the potential dependent parameters can be found in table 10.2. However, the surface charge could not be fixed because of the unreliable IS data. Thus, it was fitted for each potential separately. Nevertheless, the same cation adsorption 


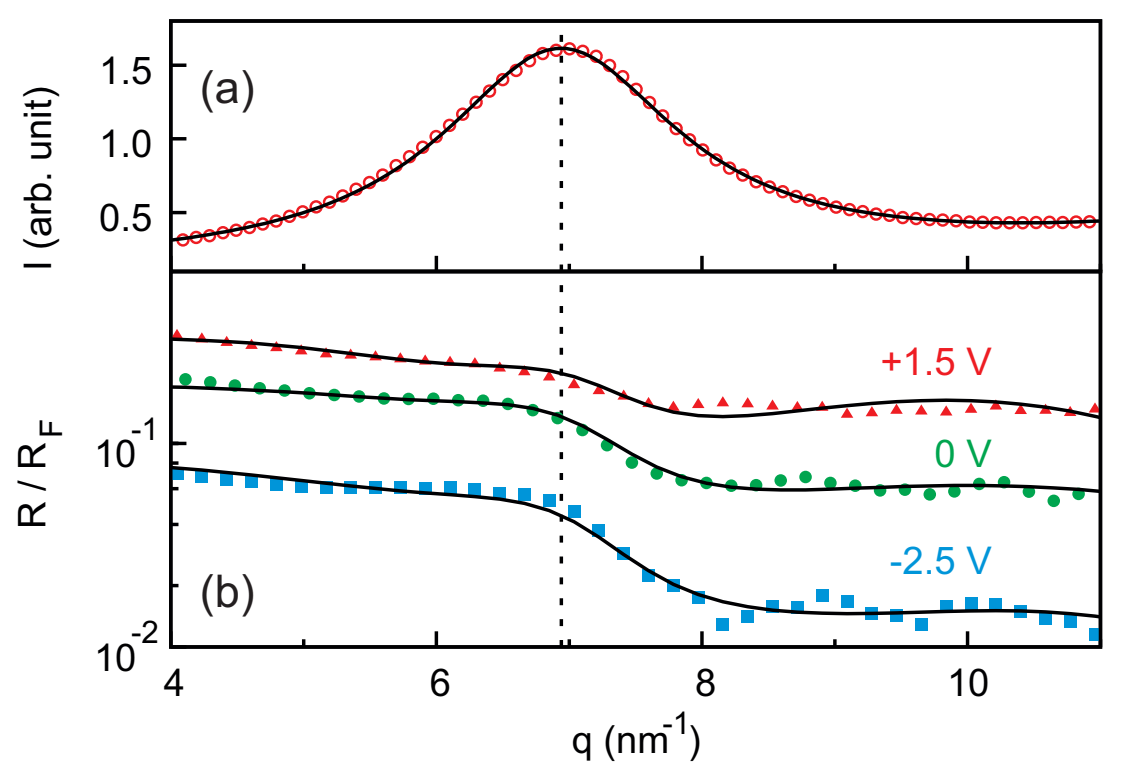

Figure 10.1: (a) Measured bulk XRD curve of $[\mathrm{bmpy}]^{+}[\mathrm{FAP}]^{-}$(red circles) and fitted Teubner-Strey model (line). The dashed vertical line indicates $q=2 \pi d_{B}^{-1}$. (b) Measured XRR curves $R(q)$ (symbols) normalized by the Fresnel reflectivity $R_{\mathrm{F}}(q)$. Lines show simultaneous fits of all three curves to the modified distorted crystal model. The $0 \mathrm{~V}$ and $-2.5 \mathrm{~V}$ curves are shifted vertically for clarity.

at $1.5 \mathrm{~V}$ is found as for the other IL. The calculated electron density profiles (figure 10.2) and the binned ion concentration (figure 10.3) are similar to the ones found for the $[\mathrm{bmpy}]^{+}[\mathrm{FAP}]^{-} /$electrode interface. The interfacial periodicity $d_{\mathrm{I}}=0.89 \mathrm{~nm}$ matches very well with the bulk periodicity $d_{\mathrm{B}}=0.91 \mathrm{~nm}$. The bulk correlation length $\xi_{\mathrm{B}}=0.91 \mathrm{~nm}$, however, is smaller than the interfacial correlation length of $\xi_{\mathrm{I}}=1.22 \mathrm{~nm}$. A reason for this discrepancy might be the generic correlations assumed in the Teubner-Strey model, that do not catch the molecular details (cf. chapter 4).

In the electron density profile (figure 10.2), the phase shift of the oscillatory profile relative to the solid electrode is clearly visible. However, the position of the first layer $z_{0}$ does not follow the same trend (table 10.2) as for the $[\mathrm{bmpy}]^{+}[\mathrm{FAP}]^{-} /$electrode interface. This can be explained considering the large size of the $[\mathrm{tba}]^{+}$cation compared to [bmpy $^{+}$. Because of its larger size, the [tba ${ }^{+}$cation occupies more volume at the interface leading to a lower maximal concentration, i.e. $\gamma>1$, and a decreased monolayer surface charge of $\sigma_{\mathrm{ML}}=16 \mu \mathrm{C} \mathrm{cm}^{-2}$. Thus, the width of the first [tba] ${ }^{+}$cation layer varies much stronger than a $[\mathrm{bmpy}]^{+}$layer for the same amount of surface charge difference. 


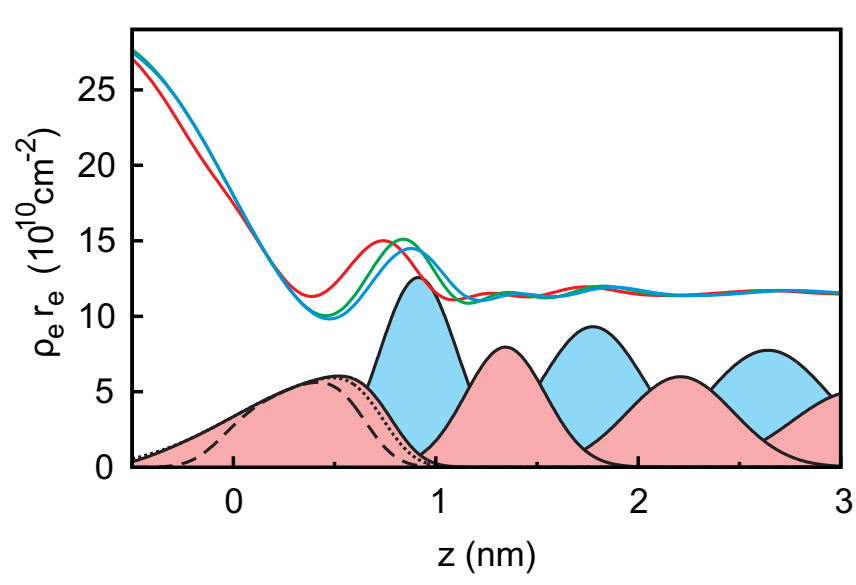

Figure 10.2: Electron density profiles extracted from the XRR curves at the potentials $1.5 \mathrm{~V}$ (red line), $0 \mathrm{~V}$ (green line) and $-2.5 \mathrm{~V}$ (blue line). The cation (light red) and anion (light blue) Gaussian distributions are plotted for a potential of $-2.5 \mathrm{~V}$. The first layer at $0 \mathrm{~V}$ (dotted black line) and at $1.5 \mathrm{~V}$ (dashed black line) are shown as well.

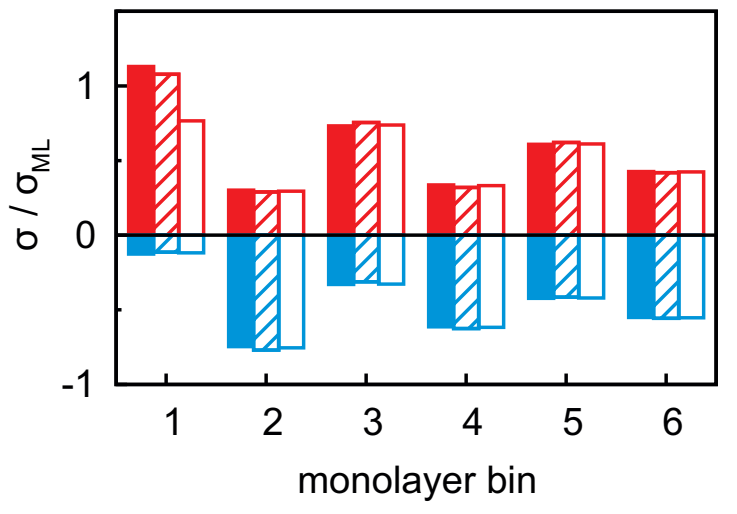

Figure 10.3: Anion (blue) and cation (red) charges $\sigma$ in the $n$ th IL layer, counted from the $[\mathrm{tba}]^{+}[\mathrm{FAP}]^{-} /$electrode interface, at potentials $-2.5 \mathrm{~V}$ (filled), $0 \mathrm{~V}$ (hatched) and $1.5 \mathrm{~V}$ (empty). Charges are normalized to the equivalent of a full-monolayer $\sigma_{\mathrm{ML}}=16 \mu \mathrm{C} \mathrm{cm}^{-2}$.

Table 10.1: $d_{\mathrm{B}}$ and $\xi_{\mathrm{B}}$ result from fits of the Teubner-Strey form to the bulk $[\mathrm{bmpy}]^{+}[\mathrm{FAP}]^{-} \mathrm{XRD}$ pattern. The potential independent model parameters result from the MDC model (cf. section 6.4). $\gamma$ is dimensionless, the other values are in $\mathrm{nm}$.

\begin{tabular}{ccccccccc}
\hline$d_{\mathrm{B}}$ & $\xi_{\mathrm{B}}$ & $d_{\mathrm{I}}$ & $\xi_{\mathrm{I}}$ & $d_{1}$ & $s_{0}^{\mathrm{c}}$ & $s_{0}^{\mathrm{a}}$ & $s_{\mathrm{WE}}$ & $\gamma$ \\
\hline 0.91 & 0.91 & 0.89 & 1.22 & 0.86 & 0.14 & 0.18 & 0.37 & 1.36 \\
\hline
\end{tabular}

Table 10.2: Potential dependent model parameters of $[\mathrm{tba}]^{+}[\mathrm{FAP}]^{-} /$electrode interface. The surface charge difference is in units of a full-monolayer $\sigma_{\mathrm{ML}}=16 \mu \mathrm{C} \mathrm{cm}^{-2}$.

\begin{tabular}{cccc}
\hline$U(\mathrm{~V})$ & $A_{\mathrm{I}} / A_{\mathrm{I}}(U=0 \mathrm{~V})$ & $z_{0}(\mathrm{~nm})$ & $\sigma / \sigma_{\mathrm{ML}}$ \\
\hline+1.5 & 0.94 & 0.33 & $43 \%$ \\
0 & 1.00 & 0.29 & $75 \%$ \\
-2.5 & 0.92 & 0.33 & $80 \%$ \\
\hline
\end{tabular}


Figure 10.4: Response of the XRR signal $R$ from the $[\mathrm{tba}]^{+}[\mathrm{FAP}]^{-} /$electrode interface to potential steps between $-2.5 \mathrm{~V}$ and $1.5 \mathrm{~V}$ at a frequency of $50 \mathrm{~Hz}$ (symbols). Solid lines are fits to the time dependence discussed in the text. Dashed lines are contributions from the second relaxation process.

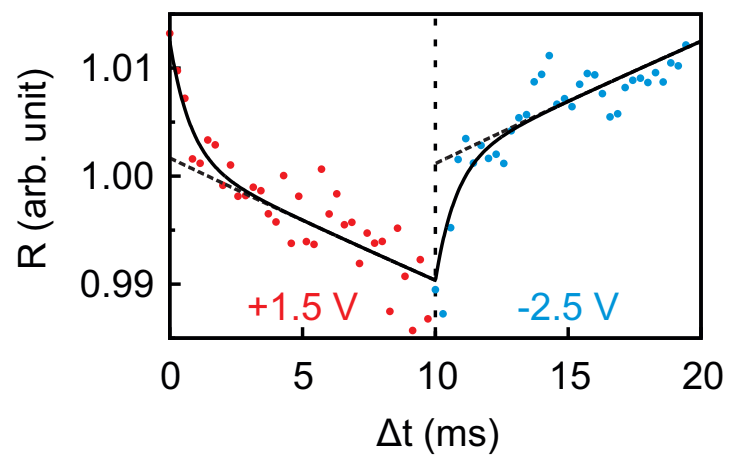

Figure 10.5: Time resolved XRR measurements at $q=4.3 \mathrm{~nm}$ from the $[\mathrm{tba}]^{+}[\mathrm{FAP}]^{-} /$electrode interface. Normalized XRR intensity $R$ (symbols, solid lines are guides to the eye) and current density $I$ (dashed curve) vs. applied potential $U$ recorded during $\mathrm{CV}$ at a scanning speed of $10 \mathrm{mV} \mathrm{s}^{-1}$. Arrows show the scan directions.

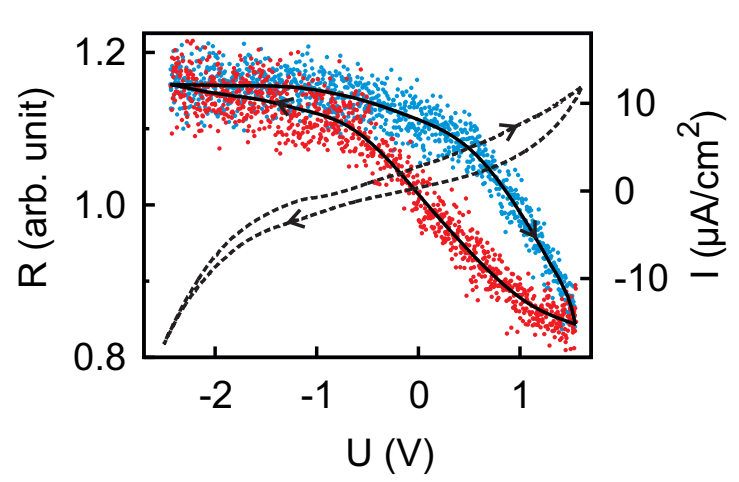

\subsection{Dynamics}

The dynamics of the $[\mathrm{tba}]^{+}[\mathrm{FAP}]^{-} /$electrode interface were studied with the same two time-resolved XRR measurements as the $[\mathrm{bmpy}]^{+}[\mathrm{FAP}]^{-} /$electrode interface. The XRR signal recorded during the abrupt switching experiments was evaluated by a fit to equation (9.2). $\quad R_{0}$ was determined from the maximal amplitude of the CV scan in figure 10.5. The fit yields a fast relaxation time $T_{1}=0.78 \pm 0.26 \mathrm{~ms}$ and a slow relaxation time $T_{2}=129 \pm 20 \mathrm{~ms}$ on the same order of magnitude than the relaxation times observed for the other IL. Also, the amplitude ratio yields the same value $R_{2} / R_{1}=14$. As the $[\mathrm{tba}]^{+}[\mathrm{FAP}]^{-}$has a similar interfacial structure like the $[\mathrm{bmpy}]^{+}[\mathrm{FAP}]^{-}$, similar dynamics can be assumed, where the fast process is again diffusion based and the slower process is associated with interfacial molecular rearrangement. The XRR signal recorded during CV (figure 10.5) shows a hysteresis loop, similar to the one in figure 9.9. This affirms the presence of ultra slow interfacial dynamics observed in $[\mathrm{bmpy}]^{+}[\mathrm{FAP}]^{-}$. The almost constant intensity between $-2.5 \mathrm{~V}$ and $0 \mathrm{~V}$ in figure 9.9 is in agreement with the only slight change of the electron density profile at those two potentials. Passing a potential of $0.5 \mathrm{~V}$, the XRR signal decreases rapidly with the increasing potential, which might be related to the pronounced reduction of cation concentration in the static electron density profile at $1.5 \mathrm{~V}$. 


\section{Chapter 11}

\section{Conclusions}

Combining electrochemical IS measurements and time resolved XRR, a comprehensive picture of the molecular-scale structure of an IL/electrode interface has been developed. Its response to applied potentials spans from a few milliseconds to hundreds of seconds. At all investigated potentials, the interface-normal ion concentration profiles exhibit a distinct layering structure. The measured XRR curves are reproduced by a single decay length and a single periodicity, independent of the applied potential. They are close to those of the bulk correlation, implying that bulk correlations dominate also the interfacial structure. The time resolved measurements suggest a three-step structure-variation scenario for the charging/discharging process at an IL/electrode interface (figure 11.1). Specifically, switching the voltage from $-2.5 \mathrm{~V}$ to $1.5 \mathrm{~V}$ reduces the surface charge by $\sim 20 \%$ of a monolayer-equivalent. The diffusion limited ion transport from and to the interface happens on a millisecond timescale. In addition, a shift occurs in the first cation layer's position relative to the electrode surface. This process, with a small capacitive strength and slow relaxation time on the order of $100 \mathrm{~ms}$, is tentatively assigned to a reorientation of substrate-adsorbed cations. Due to ion-electrode interactions, this reorientation process is strongly hindered and sensitive to electrode inhomogeneities, leading to a broad relaxation time distribution. Finally, the observations by scanning probe techniques $[41,149]$ imply that the third, even slower, $10-100$ s timescale, process observed in cyclic voltammetry is a lateral reorganization of substrate-adsorbed cations. Regrettably, this process cannot be probed directly by grazing-incidence X-ray diffraction due to the high background in such measurements at deeply-buried interfaces.

The combination of XRR and IS, providing simultaneous structural and electrochemical information, proofed to be an excellent tool to investigate electrochemical processes at interfaces. This combined approach has been steadily developed over the last years $[34,56,114,151]$ and has reached an advanced state, so that nowadays it is possible to examine interfacial structures of liquids with sub-molecular resolution. The time resolution achieved in the reported XRR experiments is exceptional for buried liquid/solid interfaces. It is over three magnitudes faster than the time resolution achieved in a recent study on a different IL/electrode system [164]. It is only comparable with 


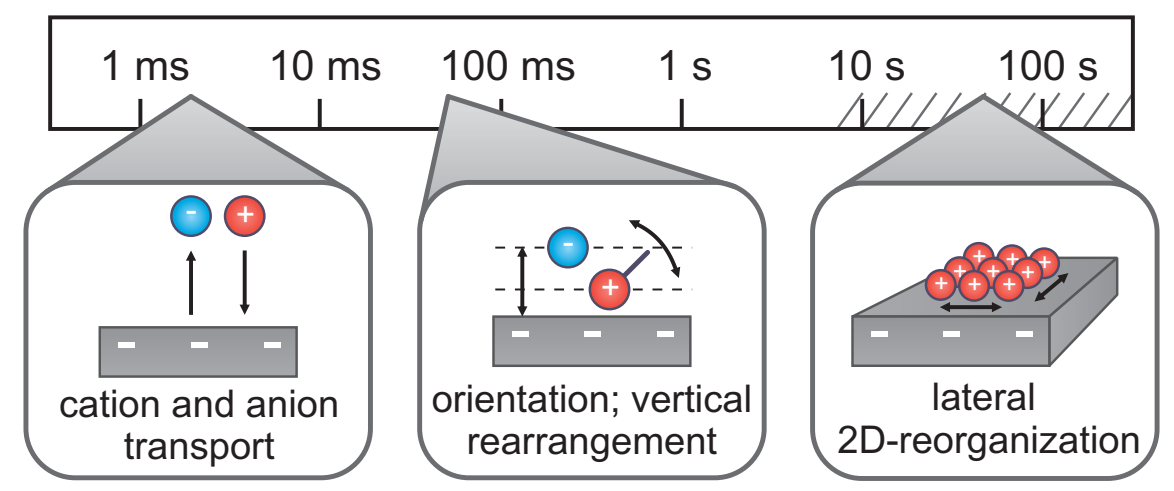

Figure 11.1: Schematic representation of the three relaxation processes during charging/discharging of the IL/electrode interface, along with their associated time scales.

studies of electrode kinetics, such as electrodeposition [151], electrode dissolution [56] or electrode reconstruction [114], where the signal is much stronger due to the high electron density of the electrode material. However, the dynamics of the mere liquid structure adjacent to the electrode has never been studied, to my knowledge, on the time scales reported in this study by means of X-ray diffraction.

This study provides a deep insight into the structure of ILs near electrodes, which is of utmost importance for numerous applications. The presented results might have considerable impact on the design of supercapacitors. A better understanding of the interfacial structure could be the basis of specific design of ILs and electrode materials in order to enhance their energy storage capacity [142]. The knowledge of interfacial processes on different time scales might enhance the control of the charging/discharging process, which is essential for operating electrical devices. Also, electroplating of metal films and nano-structured semiconductors from IL-based electrolytes might be better understood with awareness of interfacial structuring [6]. It is known that the interfacial structure in ILs has a considerable impact on the properties of the deposited film, which can be tuned by ion variation $[42,119]$. However, the ion diffusion-based deposition process is little understood.

Finally, the fundamental relation between bulk and interfacial correlations was examined in this work. In both studied ILs the correlation length and the periodicity were found to be very similar in bulk and at the interface which is in well agreement with predictions from density functional theory $[44,59]$. So far, only limited experimental evidence has been provided e.g. by studies on colloids [113] and on the free surface of liquid metals [102]. The large molecular volume of ILs and the large electron density difference between hydrocarbon-based cations and fluorinated anions makes them an excellent model system studying intrinsic correlations in liquids with X-ray scattering techniques. 


\section{Chapter 12}

\section{Other results and outlook}

Besides resolving the potential dependent interfacial structure of pure ILs, also the influence of solvents on the interfacial structure was investigated. By adding neutral solvents, the correlations of the IL-solvent mixture can be tuned reducing the strong correlations present in pure ILs. Thus, the transition between oscillatory and monotonic decaying correlations can be investigated described by the Fisher-Widom line in classical density functional theory (cf. section 2.2). Such experiments were performed in our group on solutions of $[\mathrm{bmpy}]^{+}[\mathrm{FAP}]^{-}$and propylene carbonate (PC) of different mixing rations. In a prospective publication the correlations in bulk and at a sapphire substrate will be compared [107].

In applications, ILs are often blended with organic solvents to increase their ion conductivity. The influence of the presence of solvent molecules on the interfacial dynamics was investigated in preliminary experiments. Time resolved XRR data was collected for a solution of $[\mathrm{bmpy}]^{+}[\mathrm{FAP}]^{-}$and $\mathrm{PC}$ in a 1:1 volume ratio at a BDD electrode (figure 12.1). The observed relaxation times $\tau_{1}=1.2 \mathrm{~s}$ and $\tau_{2}=11 \mathrm{~s}$ are three orders of magnitude slower than for the pure IL. This deceleration is remarkable as the relaxation time is expected to decrease with the lower viscosity of the solution. Note that the intensities at anodic and cathodic potential are inversed as compared to the pure IL, which indicates a different rearrangement of the interfacial structure of the IL in presence of PC. A possible explanation could be an interfacial adsorption process which is not governed by bulk diffusion but by a more complicated process involving both ions and solvent molecules. Also, equation (3.29) predicts an increasing relaxation time for weaker interfacial correlations. However, further experiments have to be conducted to discover the origin of these decelerated dynamics. Electrochemistry experiments could give an answer whether the diffusion process is slowed down or if the XRR signal is not sensitive on this process anymore because of the much reduced layering.

Also pulsed-field gradient NMR can provide useful information on the diffusion process in ILs. In preliminary experiments the diffusion coefficients of cations and anions in the IL $[\mathrm{bmpy}]^{+}[\mathrm{FAP}]^{-}$were determined at room temperature, yielding $D_{\mathrm{c}}=$ $5.0 \times 10^{-12} \mathrm{~m}^{2} \mathrm{~s}^{-1}$ and $D_{\mathrm{a}}=3.8 \times 10^{-12} \mathrm{~m}^{2} \mathrm{~s}^{-1}$. The calculated conductivity $\sigma_{\mathrm{L}}^{\mathrm{NMR}}=$ $2 \times 10^{-3} \mathrm{~S} \mathrm{~cm}^{-1}$ using the Nernst-Einstein relation (7.17) is one magnitude larger than 
Figure 12.1: Response of the XRR signal $R$ to potential steps between $-2.5 \mathrm{~V}$ and $1.5 \mathrm{~V}$ at a frequency of $30 \mathrm{mHz}$ (symbols) from a $1: 1[\mathrm{bmpy}]^{+}[\mathrm{FAP}]^{-}$-PC solution. Solid lines are fits to the time dependence equation (9.2). Dashed lines are contributions from the second relaxation process.

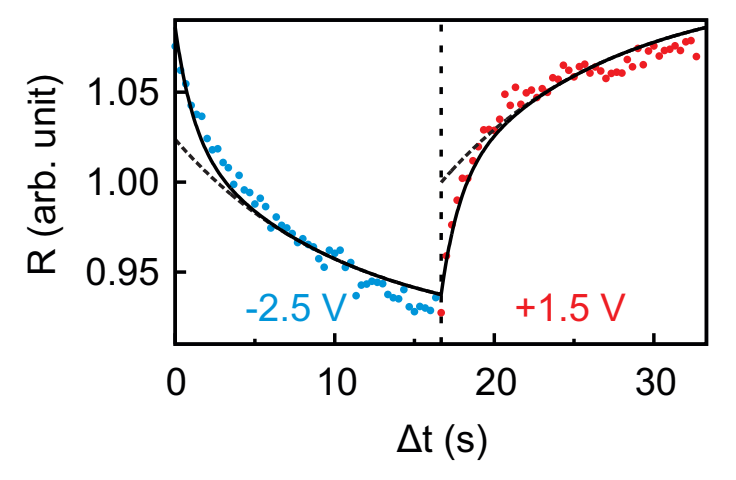

the measured conductivity $\sigma_{\mathrm{L}}^{\mathrm{IS}}=4 \times 10^{-4} \mathrm{~S} \mathrm{~cm}^{-1}$ by IS. This discrepancy was also observed in other studies [155], where it was explained with only partial dissociation of the ions. However, open questions still remain [169]. In future experiments on ILsolvent mixtures of different mixing ratios, the separate determination of the diffusion coefficients of anion, cation, and solvent molecules might help to understand the slow dynamics observed in XRR. 


\title{
Appendix A
}

\section{Abbreviations and acronyms}

\author{
AFM Atomic Force Microscope \\ BDD Boron Doped Diamond \\ bmpy 1-butyl-1-methylpyrrolidinium \\ cf. confer, compare \\ CV Cyclic Voltammetry \\ CVD Chemical Vapor Deposition \\ DSC Differential Scanning Caliometry \\ e.g. exempli gratia, for example \\ ESRF European Synchrotron Radiation Facility \\ FAP tris(perFluoroAlkyl)trifluoroPhosphate; also tris(pentafluoroethyl)trifluorophospate \\ HEMD High Energy X-ray Micro Diffraction Setup \\ i.e. it est, that is \\ IL Ionic Liquid \\ IS Impedance Spectroscopy \\ MD Molecular Dynamics \\ MDC Modified Distorted Crystal model \\ NMR Nuclear Magnetic Resonance \\ OZR Ornstein-Zernike Relation \\ PC Propylene Carbonate \\ PTFE PolyTetraFluoroEthylene \\ PZC Potential of Zero Charge \\ SAM Self Assembled Monolayer \\ SFG Sum Frequency Generation \\ STM Scanning Tunneling Microscope \\ TFSI bis(TriFluoromethylSulfonyl)Imide \\ tba tetrabutylammonium \\ vs. versus \\ XRD X-Ray Diffraction \\ XRR X-Ray Reflectivity
}




\section{Appendix B}

\section{Symbols used in equations}

\section{Elementary constants}

CODATA internationally recommended values of the fundamental physical constants where taken from NIST [154].

$\begin{array}{ll}c=2.9979 \cdot 10^{8} \mathrm{~ms}^{-1} & \text { vacuum light speed } \\ e=1.6022 \cdot 10^{-19} \mathrm{C} & \text { elementary charge } \\ \varepsilon_{0}=8.8542 \cdot 10^{-12} \mathrm{Fm}^{-1} & \text { permittivity of free space } \\ \hbar=1.0546 \cdot 10^{-34} \mathrm{JS} & \text { Planck constant } / 2 \pi \\ k_{\mathrm{B}}=1.3807 \cdot 10^{-23} \mathrm{JK}^{-1} & \text { Boltzmann constant } \\ \lambda_{\mathrm{C}}=\frac{h}{m_{\mathrm{e}} c}=2.4263 \cdot 10^{-12} \mathrm{~m} & \text { Compton wavelength of an electron } \\ m_{\mathrm{e}}=9.1094 \cdot 10^{-31} \mathrm{~kg} & \text { electron mass } \\ N_{\mathrm{A}}=6.0221 \cdot 10^{-23} \mathrm{~mol}^{-1} & \text { Avogadro constant } \\ r_{\mathrm{e}}=\frac{e^{2}}{4 \pi \epsilon_{0} m_{\mathrm{e}} c^{2}}=2.8179 \cdot 10^{-15} \mathrm{~m} & \text { classical electron radius }\end{array}$

\section{Symbols}

$\alpha$
$\alpha_{\mathrm{c}} \approx \sqrt{2 \delta}$
$\alpha_{\mathrm{f}}$
$\alpha_{\mathrm{i}}$
$A_{\mathrm{I}}$
$\beta$
$\chi^{2}$
$C$
$C_{0}$
$\Delta C$
$\hat{C}(\omega)$

Cole-Cole phase parameter

critical angle of total reflection

exit angle

incidence angle

interfacial layering amplitude

optical constant (imaginary part)

deviation between fitted and experimental data

differential capacitance

vacuum capacitance

capacitive strength

complex capacitance 


\begin{tabular}{|c|c|}
\hline$C^{\prime}(\omega)$ & real part of complex capacitance \\
\hline$C^{\prime \prime}(\omega)$ & imaginary part of complex capacitance \\
\hline$c(r)$ & direct correlation function \\
\hline$c_{\mathrm{a} / \mathrm{c}}(z)$ & anion/cation concentration \\
\hline $\bar{c}$ & mean concentration \\
\hline$c_{\alpha \beta}(r)$ & direct correlation function between species $\alpha$ and $\beta$ \\
\hline$d_{\mathrm{B}}$ & bulk periodicity \\
\hline$d_{1}$ & layer periodicity \\
\hline$d_{\mathrm{I}}$ & effective interfacial periodicity \\
\hline$\delta$ & optical constant (real part) \\
\hline$\delta(\mathbf{r})$ & Dirac delta function \\
\hline$E$ & X-ray energy \\
\hline$\varepsilon$ & dielectric constant \\
\hline$\varepsilon_{\infty}$ & dielectric constant of induced polarization \\
\hline$\hat{\varepsilon}(\omega)$ & complex dielectric function \\
\hline$\varepsilon$ & polarization vector \\
\hline$F$ & Helmholtz free energy \\
\hline$f^{0}(q)$ & angular dependent part of the atomic scattering form factor \\
\hline$f_{\alpha}(q)$ & atomic scattering form factor of element $\alpha$ \\
\hline$f^{\prime}(\omega)$ & real part of dispersion correction \\
\hline$f^{\prime \prime}(\omega)$ & imaginary part of dispersion correction \\
\hline$\gamma$ & relative maximal ion concentration at the interface \\
\hline$g(r)$ & radial distribution function \\
\hline$h(r)$ & total correlation function \\
\hline$h^{\mathrm{TS}}(r)$ & Teubner-Strey correlation function \\
\hline$h_{\alpha \beta}(r)$ & partial total correlation function \\
\hline$I^{\mathrm{S}}$ & scattered X-ray intensity \\
\hline $\mathbf{k}_{\mathrm{i}}$ & incidence wave vector \\
\hline $\mathbf{k}_{\mathrm{f}}$ & final wave vector \\
\hline$\lambda=\frac{h c}{E}$ & X-ray wavelength \\
\hline$\lambda_{\mathrm{D}}$ & Debye screening length \\
\hline$m$ & mass \\
\hline$M$ & molar mass \\
\hline$\mu_{\mathrm{abs}}$ & absorption coefficient \\
\hline$\mu$ & chemical potential \\
\hline$N$ & total number of scatterers \\
\hline$n=1-\delta+i \beta$ & complex optical constant or refractive index \\
\hline$\Delta \Omega$ & solid angle \\
\hline$\Phi_{0}$ & incident flux \\
\hline$\phi_{\alpha \beta}(r)$ & inter-particle potential \\
\hline$\psi(z)$ & electric potential \\
\hline $\mathbf{q}=\mathbf{k}_{\mathrm{f}}-\mathbf{k}_{\mathrm{i}}$ & scattering vector \\
\hline$q_{\mathrm{c}}$ & maximal wave vector transfer for total reflection \\
\hline
\end{tabular}




\begin{tabular}{|c|c|}
\hline$q_{\max }$ & maximum momentum transfer achieved in an experiment \\
\hline$q_{z}$ & $z$-component of the scattering vector \\
\hline$R\left(q_{z}\right)$ & $\mathrm{X}$-ray reflectivity \\
\hline$R_{\mathrm{F}}\left(q_{z}\right)$ & Fresnel reflectivity \\
\hline$\rho$ & scattering density \\
\hline$\rho_{\mathrm{c}}$ & charge density \\
\hline$\hat{\rho}(q)$ & Fourier transform of scattering density \\
\hline $\bar{\rho}$ & mean scattering density \\
\hline$\rho_{\mathrm{e}}$ & electron density \\
\hline$\rho_{\max }$ & maximal mass density \\
\hline$\rho_{\mathrm{m}}$ & mass density \\
\hline$\rho_{\mathrm{mol}}$ & molar density \\
\hline$\left(\frac{\mathrm{d} \sigma}{\mathrm{d} \Omega}\right)$ & differential scattering cross-section \\
\hline$\Delta \sigma(U)$ & surface charge difference \\
\hline$\sigma(U)$ & surface charge \\
\hline$\sigma_{\mathrm{ML}}$ & monolayer surface charge \\
\hline$\sigma$ & standard deviation \\
\hline$\sigma_{\mathrm{L}}$ & conductivity \\
\hline$s_{0}^{\mathrm{a}, \mathrm{c}}$ & width of Gaussian ion density distribution \\
\hline$s_{\mathrm{WE}}$ & working electrode roughness \\
\hline$S(q)$ & total liquid structure factor \\
\hline$S_{\mathrm{m}}(q)$ & mono-atomic liquid structure factor \\
\hline$S_{\alpha \beta}(q)$ & partial liquid structure factor \\
\hline$S^{\mathrm{TS}}(q)$ & Teubner-Strey structure factor \\
\hline$\tau$ & relaxation time \\
\hline$t$ & time \\
\hline$T$ & temperature \\
\hline$T_{i}$ & XRR relaxation time \\
\hline $2 \theta=\alpha_{\mathrm{i}}+\alpha_{\mathrm{f}}$ & total scattering angle \\
\hline$U$ & electrode potential \\
\hline$V_{\mathrm{M}}$ & molar volume \\
\hline$\xi_{\mathrm{B}}$ & bulk correlation length \\
\hline$\xi_{\mathrm{I}}$ & interfacial correlation length \\
\hline$Z$ & atomic number \\
\hline$z_{0}$ & position of first ion layer \\
\hline$\hat{Z}(\omega)$ & complex impedance \\
\hline$Z^{\prime}(\omega)$ & real part of complex impedance \\
\hline$Z^{\prime \prime}(\omega)$ & imaginary part of complex impedance \\
\hline
\end{tabular}




\section{Bibliography}

[1] M. T. Alam, M. M. Islam, T. Okajima, and T. Ohsaka. Electrical double layer in mixtures of room-temperature ionic liquids. The Journal of Physical Chemistry C, 113(16):6596-6601, 2009.

[2] C. Aliaga, C. S. Santos, and S. Baldelli. Surface chemistry of room-temperature ionic liquids. Physical Chemistry Chemical Physics, 9(28):3683-3700, 2007.

[3] J. Als-Nielsen and D. McMorrow. Elements of modern X-ray physics. Wiley, Hoboken, 2011.

[4] N. Anjum, M.-A. Guedeau-Boudeville, C. Stubenrauch, and A. Mourchid. Phase behavior and microstructure of microemulsions containing the hydrophobic ionic liquid 1-butyl-3-methylimidazolium hexafluorophosphate. The Journal of Physical Chemistry B, 113(1):239-244, 2009.

[5] H. V. R. Annapureddy, H. K. Kashyap, P. M. De Biase, and C. J. Margulis. What is the origin of the prepeak in the $\mathrm{X}$-ray scattering of imidazolium-based roomtemperature ionic liquids? The Journal of Physical Chemistry B, 114(50):1683816846, 2010.

[6] M. Armand, F. Endres, D. R. MacFarlane, H. Ohno, and B. Scrosati. Ionic-liquid materials for the electrochemical challenges of the future. Nature Materials, 8(8):621-629, 2009.

[7] N. W. Ashcroft and D. C. Langreth. Structure of binary liquid mixtures. i. Physical Review, 156(3):685-692, 1967.

[8] R. Atkin, N. Borisenko, M. Drüschler, S. Z. El Abedin, F. Endres, R. Hayes, B. Huber, and B. Roling. An in situ STM/AFM and impedance spectroscopy study of the extremely pure 1-butyl-1-methylpyrrolidinium tris(pentafluoroethyl)trifluorophosphate/Au(111) interface: potential dependent solvation layers and the herringbone reconstruction. Physical Chemistry Chemical Physics, 13(15):6849, 2011.

[9] R. Atkin, N. Borisenko, M. Drüschler, F. Endres, R. Hayes, B. Huber, and B. Roling. Structure and dynamics of the interfacial layer between ionic liquids and electrode materials. Journal of Molecular Liquids, 2013. 
[10] R. Atkin and G. Warr. Structure in confined room-temperature ionic liquids. Journal of Physical Chemistry C, 111(13):5162-5168, 2007.

[11] S. Baldelli. Surface structure at the ionic Liquid-Electrified metal interface. Accounts of Chemical Research, 41(3):421-431, 2008.

[12] A. J. Bard and L. R. Faulkner. Electrochemical methods: fundamentals and applications. Wiley New York, 2001.

[13] M. Bazant, B. Storey, and A. Kornyshev. Double layer in ionic liquids: Overscreening versus crowding. Physical Review Letters, 106(4), 2011.

[14] M. Z. Bazant, M. S. Kilic, B. D. Storey, and A. Ajdari. Towards an understanding of induced-charge electrokinetics at large applied voltages in concentrated solutions. Advances in Colloid and Interface Science, 152(1-2):48-88, 2009.

[15] M. Z. Bazant, K. Thornton, and A. Ajdari. Diffuse-charge dynamics in electrochemical systems. Physical Review E, 70(2):021506, 2004.

[16] J. Bikerman. XXXIX. structure and capacity of electrical double layer. Philosophical Magazine Series 7, 33(220):384-397, 1942.

[17] J. M. Black, D. Walters, A. Labuda, G. Feng, P. C. Hillesheim, S. Dai, P. T. Cummings, S. V. Kalinin, R. Proksch, and N. Balke. Bias-dependent molecularlevel structure of electrical double layer in ionic liquid on graphite. Nano Letters, 13(12):5954-5960, 2013.

[18] I. Borukhov, D. Andelman, and H. Orland. Steric effects in electrolytes: A modified poisson-boltzmann equation. Physical Review Letters, 79(3):435-438, 1997.

[19] C. J. F. Bottcher and P. Bordewijk. Dielectrics in time-dependent fields. Elsevier, 1978.

[20] I. Bou-Malham and L. Bureau. Nanoconfined ionic liquids: effect of surface charges on flow and molecular layering. Soft Matter, 6(17):4062-4065, 2010.

[21] C. M. Brett and A. M. O. Brett. Electrochemistry: principles, methods, and applications, volume 4. Oxford university press Oxford, 1993.

[22] H.-J. Butt, K. Graf, and M. Kappl. Physics and Chemistry of Interfaces. WileyVCH, Weinheim, 2003.

[23] H.-J. Butt and M. Kappl. Surface and Interfacial Forces. John Wiley \& Sons, 2009.

[24] C. Cannes, H. Cachet, C. Debiemme-Chouvy, C. Deslouis, J. de Sanoit, C. Le Naour, and V. A. Zinovyeva. Double layer at [BuMeIm][Tf2N] ionic Liquid-Pt or $-\mathrm{C}$ material interfaces. The Journal of Physical Chemistry $C$, 117(44):22915-22925, 2013. 
[25] T. Carstens, R. Hayes, S. Z. E. Abedin, B. Corr, G. B. Webber, N. Borisenko, R. Atkin, and F. Endres. In situ STM, AFM and DTS study of the interface 1hexyl-3-methylimidazolium tris(pentafluoroethyl)trifluorophosphate/Au(l 11 1). Electrochimica Acta, 82:48-59, 2012.

[26] D. L. Chapman. A contribution to the theory of electrocapillarity. Philosophical magazine, 25(148):475-481, 1913.

[27] R. Coelho. Sur la relaxation d'une charge d'espace. Revue de Physique Appliquée, 18(3):137-146, 1983.

[28] R. Coelho. On the static permittivity of dipolar and conductive media - an educational approach. Journal of Non-Crystalline Solids, 131-133, Part 2:11361139, 1991.

[29] K. S. Cole and R. H. Cole. Dispersion and absorption in dielectrics i. alternating current characteristics. The Journal of Chemical Physics, 9(4):341-351, 1941.

[30] R. Costa, C. M. Pereira, and F. Silva. Electric double layer studies at the interface of mercury-binary ionic liquid mixtures with a common anion. RSC Advances, 3(29):11697-11706, 2013.

[31] Q. Dou, M. Sha, H. Fu, and G. Wu. Mass distribution and diffusion of [1-butyl-3methylimidazolium][y] ionic liquids adsorbed on the graphite surface at 300-800 k. ChemPhysChem, 11(11):2438-2443, 2010.

[32] M. Drüschler, N. Borisenko, J. Wallauer, C. Winter, B. Huber, F. Endres, and B. Roling. New insights into the interface between a single-crystalline metal electrode and an extremely pure ionic liquid: slow interfacial processes and the influence of temperature on interfacial dynamics. Physical Chemistry Chemical Physics, 14(15):5090-5099, 2012.

[33] M. Drüschler, B. Huber, and B. Roling. On capacitive processes at the interface between 1-ethyl-3-methylimidazolium tris(pentafluoroethyl)trifluorophosphate and au(111). J. Phys. Chem. C, 115(14):6802-6808, 2011.

[34] J. F. L. Duval, S. Bera, L. J. Michot, J. Daillant, L. Belloni, O. Konovalov, and D. Pontoni. X-ray reflectivity at polarized liquid-Hg-Aqueous-Electrolyte interface: Challenging macroscopic approaches for ion-specificity issues. Physical Review Letters, 108(20):206102, 2012.

[35] J. C. Dyre. The random free-energy barrier model for ac conduction in disordered solids. Journal of Applied Physics, 64(5):2456-2468, 1988.

[36] A. Efimova, G. Hubrig, and P. Schmidt. Thermal stability and crystallization behavior of imidazolium halide ionic liquids. Thermochimica Acta, 573:162$169,2013$. 
[37] M. Eigen and E. Wicke. Zur theorie der starken elektrolyte. Naturwissenschaften, 38(19):453-454, 1951.

[38] M. Eigen and E. Wicke. The thermodynamics of electrolytes at higher concentration. The Journal of Physical Chemistry, 58(9):702-714, 1954.

[39] A. Elsen, B. M. Murphy, B. M. Ocko, L. Tamam, M. Deutsch, I. Kuzmenko, and O. M. Magnussen. Surface layering at the mercury-electrolyte interface. Physical Review Letters, 104(10):105501, 2010.

[40] S. Emmert, M. Wolf, R. Gulich, S. Krohns, S. Kastner, P. Lunkenheimer, and A. Loidl. Electrode polarization effects in broadband dielectric spectroscopy. The European Physical Journal B, 83(2):157-165, 2011.

[41] F. Endres, N. Borisenko, S. Z. E. Abedin, R. Hayes, and R. Atkin. The interface ionic liquid(s)/electrode(s): In situ STM and AFM measurements. Faraday Discussions, 154(0):221-233, 2011.

[42] F. Endres, O. Höfft, N. Borisenko, L. H. Gasparotto, A. Prowald, R. Al-Salman, T. Carstens, R. Atkin, A. Bund, and S. Z. E. Abedin. Do solvation layers of ionic liquids influence electrochemical reactions? Physical Chemistry Chemical Physics, 12(8):1724-1732, Feb. 2010.

[43] J. M. S. S. Esperança, H. J. R. Guedes, M. Blesic, and L. P. N. Rebelo. Densities and derived thermodynamic properties of ionic liquids. 3. phosphonium-based ionic liquids over an extended pressure range. Journal of Chemical \& Engineering Data, 51(1):237-242, 2006.

[44] R. Evans and R. J. F. Leote de Carvalho. Decay of correlations in bulk fluids and at interfaces: A density-functional perspective. In B. B. Laird, R. B. Ross, and T. Ziegler, editors, Chemical Applications of Density-Functional Theory, volume 629, pages 166-184. American Chemical Society, Washington, DC, 1996.

[45] T. E. Faber and J. M. Ziman. A theory of the electrical properties of liquid metals. Philosophical Magazine, 11(109):153-173, 1965.

[46] M. Fedorov, N. Georgi, and A. Kornyshev. Double layer in ionic liquids: The nature of the camel shape of capacitance. Electrochemistry Communications, 12(2):296-299, 2010.

[47] M. V. Fedorov and A. A. Kornyshev. Towards understanding the structure and capacitance of electrical double layer in ionic liquids. Electrochimica Acta, 53(23):6835-6840, 2008.

[48] M. V. Fedorov and A. A. Kornyshev. Ionic liquids at electrified interfaces. Chemical Reviews, 114(5):2978-3036, 2014. 
[49] H. E. Fischer, A. C. Barnes, and P. S. Salmon. Neutron and x-ray diffraction studies of liquids and glasses. Reports on Progress in Physics, 69(1):233-299, 2006.

[50] M. E. Fisher and B. Wiodm. Decay of correlations in linear systems. The Journal of Chemical Physics, 50(9):3756-3772, 1969.

[51] V. Freise. Zur theorie der diffusen doppelschicht. Zeitschrift Fur Elektrochemie, 56(8):822-827, 1952. WOS:A1952UQ19800025.

[52] N. Georgi, A. A. Kornyshev, and M. V. Fedorov. The anatomy of the double layer and capacitance in ionic liquids with anisotropic ions: Electrostriction vs. lattice saturation. Journal of Electroanalytical Chemistry, 649(1-2):261-267, 2010.

[53] M. Gnahm, C. Berger, M. Arkhipova, H. Kunkel, T. Pajkossy, G. Maas, and D. M. Kolb. The interfaces of au(111) and au(100) in a hexaalkyl-substituted guanidinium ionic liquid: an electrochemical and in situ STM study. Physical Chemistry Chemical Physics, 14(30):10647-10652, 2012.

[54] M. Gnahm, C. Müller, R. Répánszki, T. Pajkossy, and D. M. Kolb. The interface between au(100) and 1-butyl-3-methyl-imidazolium-hexafluorophosphate. Physical Chemistry Chemical Physics, 13(24):11627-11633, 2011.

[55] M. Gnahm, T. Pajkossy, and D. Kolb. The interface between au(1 111$)$ and an ionic liquid. Electrochimica Acta, 55(21):6212-6217, 2010.

[56] F. Golks, K. Krug, Y. Gründer, J. Zegenhagen, J. Stettner, and O. M. Magnussen. High-speed in situ surface x-ray diffraction studies of the electrochemical dissolution of au(001). J. Am. Chem. Soc., 133(11):3772-3775, 2011.

[57] M. Gouy. Sur la constitution de la charge électrique à la surface d'un électrolyte. Journal de Physique Théorique et Appliquée, 9(1):457-468, 1910.

[58] D. C. Grahame. The electrical double layer and the theory of electrocapillarity. Chemical Reviews, 41(3):441-501, 1947.

[59] C. Grodon, M. Dijkstra, R. Evans, and R. Roth. Homogeneous and inhomogeneous hard-sphere mixtures: manifestations of structural crossover. Molecular physics, 103(21-23):3009-3023, 2005.

[60] J. Guo, G. A. Baker, P. C. Hillesheim, S. Dai, R. W. Shaw, and S. M. Mahurin. Fluorescence correlation spectroscopy evidence for structural heterogeneity in ionic liquids. Physical Chemistry Chemical Physics, 13(27):12395-12398, 2011.

[61] T. Hahn, U. Shmueli, A. J. C. Wilson, and E. Prince. International tables for crystallography. D. Reidel Publishing Company, 2005. 
[62] J.-P. Hansen and I. R. McDonald. Theory of simple liquids. Access Online via Elsevier, 1990.

[63] C. Hardacre, J. D. Holbrey, C. L. Mullan, T. G. A. Youngs, and D. T. Bowron. Small angle neutron scattering from 1-alkyl-3-methylimidazolium hexafluorophosphate ionic liquids ([cnmim] $[\mathrm{PF} 6], \mathrm{n}=4,6$, and 8). The Journal of Chemical Physics, 133(7):074510, 2010.

[64] R. Hayes, N. Borisenko, M. K. Tam, P. C. Howlett, F. Endres, and R. Atkin. Double layer structure of ionic liquids at the au(111) electrode interface: An atomic force microscopy investigation. The Journal of Physical Chemistry C, 115(14):6855-6863, 2011.

[65] Z. Hu, J. Vatamanu, O. Borodin, and D. Bedrov. A molecular dynamics simulation study of the electric double layer and capacitance of [BMIM][PF6] and [BMIM][BF4] room temperature ionic liquids near charged surfaces. Physical Chemistry Chemical Physics, 15(34):14234-14247, 2013.

[66] B. Huber and B. Roling. Development of a Ag/Ag+ micro-reference electrode for electrochemical measurements in ionic liquids. Electrochimica Acta, 56(19):6569-6572, 2011.

[67] V. M. Hultgren, A. W. A. Mariotti, A. M. Bond, and A. G. Wedd. Reference potential calibration and voltammetry at macrodisk electrodes of metallocene derivatives in the ionic liquid [bmim][PF6]. Analytical Chemistry, 74(13):31513156, 2002.

[68] J. Hunger, A. Stoppa, S. Schrödle, G. Hefter, and R. Buchner. Temperature dependence of the dielectric properties and dynamics of ionic liquids. ChemPhysChem, 10(4):723-733, 2009.

[69] B.-R. Hyun, S. V. Dzyuba, R. A. Bartsch, and E. L. Quitevis. Intermolecular dynamics of room-temperature ionic liquids: femtosecond optical kerr effect measurements on 1-alkyl-3-methylimidazolium bis((trifluoromethyl)sulfonyl)imides $\dagger$. The Journal of Physical Chemistry A, 106(33):7579-7585, 2002.

[70] N. V. Ignat'ev, U. Welz-Biermann, A. Kucheryna, G. Bissky, and H. Willner. New ionic liquids with tris (perfluoroalkyl) trifluorophosphate (FAP) anions. Journal of Fluorine Chemistry, 126(8):1150-1159, 2005.

[71] M. M. Islam, M. T. Alam, and T. Ohsaka. Electrical double-layer structure in ionic liquids: A corroboration of the theoretical model by experimental results. The Journal of Physical Chemistry C, 112(42):16568-16574, 2008. 
[72] M. M. Islam, M. T. Alam, T. Okajima, and T. Ohsaka. Electrical double layer structure in ionic liquids: An understanding of the unusual Capacitance-Potential curve at a nonmetallic electrode. J. Phys. Chem. C, 113(9):3386-3389, 2009.

[73] K. Iwata, H. Okajima, S. Saha, and H.-o. Hamaguchi. Local structure formation in alkyl-imidazolium-based ionic liquids as revealed by linear and nonlinear raman spectroscopy. Accounts of Chemical Research, 40(11):1174-1181, 2007.

[74] K. Izutsu. Electrochemistry in nonaqueous solutions. John Wiley \& Sons, 2009.

[75] D.-e. Jiang, D. Meng, and J. Wu. Density functional theory for differential capacitance of planar electric double layers in ionic liquids. Chemical Physics Letters, 504(4-6):153-158, 2011.

[76] W. L. Jorgensen, D. S. Maxwell, and J. Tirado-Rives. Development and testing of the OPLS all-atom force field on conformational energetics and properties of organic liquids. Journal of the American Chemical Society, 118(45):1122511236, 1996.

[77] A. Kaintz, G. Baker, A. Benesi, and M. Maroncelli. Solute diffusion in ionic liquids, NMR measurements and comparisons to conventional solvents. The Journal of Physical Chemistry B, 117(39):11697-11708, 2013.

[78] H. K. Kashyap, J. J. Hettige, H. V. R. Annapureddy, and C. J. Margulis. SAXS anti-peaks reveal the length-scales of dual positive-negative and polar-apolar ordering in room-temperature ionic liquids. Chemical Communications, 48(42):5103-5105, 2012.

[79] H. K. Kashyap, C. S. Santos, H. V. R. Annapureddy, N. S. Murthy, C. J. Margulis, and J. Edward W. Castner. Temperature-dependent structure of ionic liquids: Xray scattering and simulations. Faraday Discussions, 154(0):133-143, 2011.

[80] H. K. Kashyap, C. S. Santos, N. S. Murthy, J. J. Hettige, K. Kerr, S. Ramati, J. Gwon, M. Gohdo, S. I. Lall-Ramnarine, J. F. Wishart, C. J. Margulis, and E. W. Castner. Structure of 1-alkyl-1-methylpyrrolidinium bis(trifluoromethylsulfonyl)amide ionic liquids with linear, branched, and cyclic alkyl groups. The Journal of Physical Chemistry B, 117(49):15328-15337, 2013.

[81] D. A. Keen. A comparison of various commonly used correlation functions for describing total scattering. Journal of Applied Crystallography, 34(2):172-177, 2001.

[82] M. S. Kilic, M. Z. Bazant, and A. Ajdari. Steric effects in the dynamics of electrolytes at large applied voltages. i. double-layer charging. Physical Review E, 75(2):021502, 2007. 
[83] M. S. Kilic, M. Z. Bazant, and A. Ajdari. Steric effects in the dynamics of electrolytes at large applied voltages. II. modified poisson-nernst-planck equations. Physical Review E, 75(2):021503, 2007.

[84] J. G. Kirkwood. Statistical mechanics of liquid solutions. Chemical Reviews, 19(3):275-307, 1936.

[85] R. J. Klein, S. Zhang, S. Dou, B. H. Jones, R. H. Colby, and J. Runt. Modeling electrode polarization in dielectric spectroscopy: Ion mobility and mobile ion concentration of single-ion polymer electrolytes. The Journal of Chemical Physics, 124(14):144903, 2006.

[86] A. A. Kornyshev. Double-layer in ionic liquids: paradigm change? The Journal of Physical Chemistry B, 111(20):5545-5557, 2007.

[87] C. Krause, J. R. Sangoro, C. Iacob, and F. Kremer. Charge transport and dipolar relaxations in imidazolium-based ionic liquids. The Journal of Physical Chemistry B, 114(1):382-386, 2010.

[88] F. Kremer and A. Schönhals. Broadband Dielectric Spectroscopy. Springer, 2003.

[89] S. Lamperski, M. Kaja, L. B. Bhuiyan, J. Wu, and D. Henderson. Influence of anisotropic ion shape on structure and capacitance of an electric double layer: A monte carlo and density functional study. The Journal of Chemical Physics, 139(5):054703, 2013.

[90] Y. Lauw, T. Rodopoulos, M. Gross, A. Nelson, R. Gardner, and M. D. Horne. Electrochemical cell for neutron reflectometry studies of the structure of ionic liquids at electrified interface. Review of Scientific Instruments, 81(7):074101, 2010 .

[91] R. Leote de Carvalho and R. Evans. The decay of correlations in ionic fluids. Molecular Physics, 83(4):619-654, 1994.

[92] A. Lewandowski, T. Majkowski, and M. Galinski. Electrocapillary curves for the Hg/Ionic liquid interface. Zeitschrift für Naturforschung-A, 64(3):263, 2009.

[93] S. Li, J. L. Bañuelos, J. Guo, L. Anovitz, G. Rother, R. W. Shaw, P. C. Hillesheim, S. Dai, G. A. Baker, and P. T. Cummings. Alkyl chain length and temperature effects on structural properties of pyrrolidinium-based ionic liquids: A combined atomistic simulation and small-angle x-ray scattering study. The Journal of Physical Chemistry Letters, 3(1):125-130, 2012.

[94] J. E. Lind, H. A. A. Abdel-Rehim, and S. W. Rudich. Structure of organic melts1. J. Phys. Chem., 70(11):3610-3619, 1966. 
[95] V. Lockett, M. Horne, R. Sedev, T. Rodopoulos, and J. Ralston. Differential capacitance of the double layer at the electrode/ionic liquids interface. Phys. Chem. Chem. Phys., 12(39):12499-12512, 2010.

[96] V. Lockett, R. Sedev, J. Ralston, M. Horne, and T. Rodopoulos. Differential capacitance of the electrical double layer in imidazolium-based ionic liquids: Influence of potential, cation size, and temperature. J. Phys. Chem. C, 112(19):74867495, 2008.

[97] J. Lyklema. Fundamentals of Interface and Colloid Science: Solid-Liquid Interfaces. Academic Press, 1995.

[98] R. M. Lynden-Bell, M. G. Del Pópolo, T. G. A. Youngs, J. Kohanoff, C. G. Hanke, J. B. Harper, and C. C. Pinilla. Simulations of ionic liquids, solutions, and surfaces. Accounts of Chemical Research, 40(11):1138-1145, 2007.

[99] R. M. Lynden-Bell, A. I. Frolov, and M. V. Fedorov. Electrode screening by ionic liquids. Phys. Chem. Chem. Phys., 14(8):2693-2701, 2012.

[100] J. R. Macdonald. Theory of ac space-charge polarization effects in photoconductors, semiconductors, and electrolytes. Physical Review, 92(1):4-17, 1953.

[101] J. R. MaCdonald. Double layer capacitance and relaxation in electrolytes and solids. Transactions of the Faraday Society, 66(0):943-958, 1970.

[102] O. M. Magnussen, B. M. Ocko, M. J. Regan, K. Penanen, P. S. Pershan, and M. Deutsch. X-ray reflectivity measurements of surface layering in liquid mercury. Physical Review Letters, 74(22):4444-4447, 1995.

[103] C. Merlet, M. Salanne, and B. Rotenberg. New coarse-grained models of imidazolium ionic liquids for bulk and interfacial molecular simulations. The Journal of Physical Chemistry C, 116(14):7687-7693, 2012.

[104] L. Mesin and M. Scalerandi. Effects of transducer size on impedance spectroscopy measurements. Physical Review E, 85(5):051505, 2012.

[105] M. Mezger. X-ray Studies of the Density Depletion at Hydrophobic Water-Solid Interfaces. PhD thesis, Universität Stuttgart, Stuttgart, 2008.

[106] M. Mezger, B. M. Ocko, H. Reichert, and M. Deutsch. Surface layering and melting in an ionic liquid studied by resonant soft x-ray reflectivity. Proceedings of the National Academy of Sciences, 110(10):3733-3737, 2013. PMID: 23431181.

[107] M. Mezger, R. Roth, H. Schröder, P. Reichert, D. Pontoni, and H. Reichert. The solid-liquid interface of ionic liquid solutions - interfacial layering and solventinduced screening. in preparation. 
[108] M. Mezger, S. Schramm, H. Schroder, H. Reichert, M. Deutsch, E. J. De Souza, J. S. Okasinski, B. M. Ocko, V. Honkimaki, and H. Dosch. Layering of [BMIM][sup +]-based ionic liquids at a charged sapphire interface. The Journal of Chemical Physics, 131(9):094701, 2009.

[109] M. Mezger, H. Schroder, H. Reichert, S. Schramm, J. S. Okasinski, S. Schoder, V. Honkimaki, M. Deutsch, B. M. Ocko, J. Ralston, M. Rohwerder, M. Stratmann, and H. Dosch. Molecular layering of fluorinated ionic liquids at a charged sapphire (0001) surface. Science, 322(5900):424-428, 2008.

[110] M. Mezger, S. Schöder, H. Reichert, H. Schröder, J. Okasinski, V. Honkimaki, J. Ralston, J. Bilgram, R. Roth, and H. Dosch. Water and ice in contact with octadecyl-trichlorosilane functionalized surfaces: A high resolution $\mathrm{x}$-ray reflectivity study. The Journal of Chemical Physics, 128(24):244705, 2008.

[111] C. Nanjundiah, S. F. McDevitt, and V. R. Koch. Differential capacitance measurements in solvent-free ionic liquids at hg and c interfaces. Journal of The Electrochemical Society, 144(10):3392-3397, 1997.

[112] N. Nishi, Y. Hirano, T. Motokawa, and T. Kakiuchi. Ultraslow relaxation of the structure at the ionic liquid|gold electrode interface to a potential step probed by electrochemical surface plasmon resonance measurements: asymmetry of the relaxation time to the potential-step direction. Physical Chemistry Chemical Physics, 15(28):11615-11619, 2013.

[113] K. Nygård and O. Konovalov. Decay of interfacial fluid ordering probed by x-ray reflectivity. Soft Matter, 8(19):5180-5186, 2012.

[114] B. M. Ocko, J. Wang, A. Davenport, and H. Isaacs. In situ X-ray reflectivity and diffraction studies of the au(001) reconstruction in an electrochemical cell. Physical Review Letters, 65(12):1466, 1990.

[115] L. Ornstein and F. Zernike. Die linearen dimensionen der dichteschwankungen. Phys. Zeit, 19:134-7, 1918.

[116] T. Pajkossy and D. M. Kolb. The interfacial capacitance of au(100) in an ionic liquid, 1-butyl-3-methyl-imidazolium hexafluorophosphate. Electrochemistry Communications, 13(3):284-286, 2011.

[117] L. G. Parratt. Surface studies of solids by total reflection of x-rays. Physical Review, 95(2):359-369, 1954.

[118] C. J. Pings and J. Waser. Analysis of scattering data for mixtures of amorphous solids or liquids. The Journal of Chemical Physics, 48(7):3016-3018, 1968. 
[119] G. Pulletikurthi, A. Lahiri, T. Carstens, N. Borisenko, S. Z. E. Abedin, and F. Endres. Electrodeposition of silicon from three different ionic liquids: possible influence of the anion on the deposition process. Journal of Solid State Electrochemistry, 17(11):2823-2832, Nov. 2013.

[120] M. J. Regan, E. H. Kawamoto, S. Lee, P. S. Pershan, N. Maskil, M. Deutsch, O. M. Magnussen, B. M. Ocko, and L. E. Berman. Surface layering in liquid gallium: An x-ray reflectivity study. Physical Review Letters, 75(13):2498-2501, 1995.

[121] P. Reichert, K. Skov Kjaer, T. B. van Driel, J. W. Ochsmann, M. Deutsch, D. Pontoni, M. M. Nielsen, and M. Mezger. Watching a capacitor at work - molecular scale structure and dynamics at an ionic Liquid/Electrode interface. in preparation.

[122] M. G. C. Resende. http://www2.research.att.com/ $/ \mathrm{mgcr} /$.

[123] M. G. C. Resende, R. F. Toso, J. F. Gonçalves, and R. M. A. Silva. A biased random-key genetic algorithm for the steiner triple covering problem. Optimization Letters, 6(4):605-619, 2012.

[124] C. E. Resende Prado and L. C. Gomide Freitas. Molecular dynamics simulation of the room-temperature ionic liquid 1-butyl-3-methylimidazolium tetrafluoroborate. Journal of Molecular Structure: THEOCHEM, 847(1-3):93-100, 2007.

[125] M. C. C. Ribeiro. Intermolecular vibrations and fast relaxations in supercooled ionic liquids. The Journal of Chemical Physics, 134(24):244507, 2011.

[126] B. Roling, M. Drüschler, and B. Huber. Slow and fast capacitive process taking place at the ionic liquid/electrode interface. Faraday Discussions, 154:303, 2012.

[127] M. Rovere and M. P. Tosi. Structure and dynamics of molten salts. Reports on Progress in Physics, 49(9):1001, 1986.

[128] O. Russina, A. Triolo, L. Gontrani, R. Caminiti, D. Xiao, L. G. H. Jr, R. A. Bartsch, E. L. Quitevis, N. Plechkova, and K. R. Seddon. Morphology and intermolecular dynamics of 1-alkyl-3-methylimidazolium bis $\{$ (trifluoromethane)sulfonyl $\}$ amide ionic liquids: structural and dynamic evidence of nanoscale segregation. Journal of Physics: Condensed Matter, 21(42):424121, 2009.

[129] P. S. Salmon. Decay of the pair correlations and small-angle scattering for binary liquids and glasses. Journal of Physics: Condensed Matter, 18(50):11443, 2006.

[130] J. R. Sangoro, C. Iacob, S. Naumov, R. Valiullin, H. Rexhausen, J. Hunger, R. Buchner, V. Strehmel, J. Kärger, and F. Kremer. Diffusion in ionic liquids: the interplay between molecular structure and dynamics. Soft Matter, 7(5):16781681, 2011. 
[131] J. R. Sangoro, A. Serghei, S. Naumov, P. Galvosas, J. Kärger, C. Wespe, F. Bordusa, and F. Kremer. Charge transport and mass transport in imidazolium-based ionic liquids. Physical Review E, 77(5):051202, 2008.

[132] C. S. Santos, H. V. R. Annapureddy, N. S. Murthy, H. K. Kashyap, E. W. C. Jr, and C. J. Margulis. Temperature-dependent structure of methyltributylammonium bis(trifluoromethylsulfonyl)amide: $\mathrm{X}$ ray scattering and simulations. The Journal of Chemical Physics, 134(6):064501, 2011.

[133] C. S. Santos, N. S. Murthy, G. A. Baker, and E. W. C. Jr. Communication: X-ray scattering from ionic liquids with pyrrolidinium cations. The Journal of Chemical Physics, 134(12):121101, 2011.

[134] H. Schroder. Molecular ordering of ionic liquids at a sapphire hard wall A high energy $x$-ray reflectivity study. PhD thesis, Universität Stuttgart, Stuttgart, 2009.

[135] K.-V. Schubert and R. Strey. Small-angle neutron scattering from microemulsions near the disorder line in water/formamide-octane-CiEj systems. The Journal of Chemical Physics, 95(11):8532-8545, 1991.

[136] Selimar Rivera-Rubero and Steven Baldelli. Sum frequency generation spectroscopy and electrochemical analysis of the 1-butyl-3-methylimidazolium bis $\{$ (trifluoromethyl)sulfonyl $\}$ amide double layer structure on the platinum electrode. In Ionic Liquids: From Knowledge to Application, volume 1030 of ACS Symposium Series, pages 291-304. American Chemical Society, 2009.

[137] A. Serghei, M. Tress, J. R. Sangoro, and F. Kremer. Electrode polarization and charge transport at solid interfaces. Physical Review B, 80(18):184301, 2009.

[138] M. Sha, G. Wu, Q. Dou, Z. Tang, and H. Fang. Double-layer formation of [bmim][PF6] ionic liquid triggered by surface negative charge. Langmuir, 26(15):12667-12672, 2010.

[139] R. D. Shannon. Revised effective ionic radii and systematic studies of interatomic distances in halides and chalcogenides. Acta Crystallographica Section A, 32(5):751-767, 1976.

[140] S. Shigeto and H.-o. Hamaguchi. Evidence for mesoscopic local structures in ionic liquids: CARS signal spatial distribution of cnmim[PF6] $(\mathrm{n}=4,6,8)$. Chemical Physics Letters, 427(4-6):329-332, 2006.

[141] X. Si, S. Li, Y. Wang, S. Ye, and T. Yan. Effects of specific adsorption on the differential capacitance of imidazolium-based ionic liquid electrolytes. ChemPhysChem, 13(7):1671-1676, 2012.

[142] P. Simon and Y. Gogotsi. Materials for electrochemical capacitors. Nature Materials, 7(11):845-854, 2008. 
[143] R. Singh Payal and S. Balasubramanian. Orientational ordering of ionic liquids near a charged mica surface. ChemPhysChem, 13(7):1764-1771, 2012.

[144] L. J. A. Siqueira and M. C. C. Ribeiro. Charge ordering and intermediate range order in ammonium ionic liquids. The Journal of Chemical Physics, 135(20):204506, 2011.

[145] L. B. Skinner, C. J. Benmore, and J. B. Parise. Area detector corrections for high quality synchrotron X-ray structure factor measurements. Nuclear Instruments and Methods in Physics Research Section A: Accelerators, Spectrometers, Detectors and Associated Equipment, 662(1):61-70, 2012.

[146] X. Song, H. Hamano, B. Minofar, R. Kanzaki, K. Fujii, Y. Kameda, S. Kohara, M. Watanabe, S.-i. Ishiguro, and Y. Umebayashi. Structural heterogeneity and unique distorted hydrogen bonding in primary ammonium nitrate ionic liquids studied by high-energy x-ray diffraction experiments and MD simulations. The Journal of Physical Chemistry B, 116(9):2801-2813, 2012.

[147] O. Stern. The theory of the electrolytic double shift. Zeitschrift für Elektrochemie und angewandte physikalische Chemie, 30:508-516, 1924.

[148] G. R. Strobl. The physics of polymers: concepts for understanding their structures and behavior. Springer, 2007.

[149] Y.-Z. Su, Y.-C. Fu, J.-W. Yan, Z.-B. Chen, and B.-W. Mao. Double layer of au(100)/Ionic liquid interface and its stability in imidazolium-based ionic liquids. Angewandte Chemie International Edition, 48(28):5148-5151, 2009.

[150] L. Tamam, B. M. Ocko, H. Reichert, and M. Deutsch. Checkerboard selfpatterning of an ionic liquid film on mercury. Physical Review Letters, 106(19):197801, 2011.

[151] K. Tamura, J. X. Wang, R. R. Adžic, and B. M. Ocko. Kinetics of monolayer bi electrodeposition on au(111): Surface X-ray scattering and current transients. The Journal of Physical Chemistry B, 108(6):1992-1998, 2004.

[152] Z. L. Terranova and S. A. Corcelli. On the mechanism of solvation dynamics in imidazolium-based ionic liquids. The Journal of Physical Chemistry B, 117(49):15659-15666, 2013.

[153] M. Teubner and R. Strey. Origin of the scattering peak in microemulsions. The Journal of Chemical Physics, 87(5):3195-3200, 1987.

[154] u. The nist reference on constants and uncertainty. http://physics.nist.gov/cuu/Constants/. 
[155] H. Tokuda, K. Hayamizu, K. Ishii, M. A. B. H. Susan, and M. Watanabe. Physicochemical properties and structures of room temperature ionic liquids. 1. variation of anionic species. The Journal of Physical Chemistry B, 108(42):16593-16600, 2004.

[156] H. Tokuda, K. Hayamizu, K. Ishii, M. A. B. H. Susan, and M. Watanabe. Physicochemical properties and structures of room temperature ionic liquids. 2. variation of alkyl chain length in imidazolium cation. The Journal of Physical Chemistry $B, 109(13): 6103-6110,2005$.

[157] H. Tokuda, K. Ishii, M. A. B. H. Susan, S. Tsuzuki, K. Hayamizu, and M. Watanabe. Physicochemical properties and structures of room-temperature ionic liquids. 3. variation of cationic structures. The Journal of Physical Chemistry B, 110(6):2833-2839, 2006.

[158] H. Tokuda, S. Tsuzuki, M. A. B. H. Susan, K. Hayamizu, and M. Watanabe. How ionic are room-temperature ionic liquids? an indicator of the physicochemical properties. The Journal of Physical Chemistry B, 110(39):19593-19600, 2006.

[159] M. Tolan. X-ray scattering from soft-matter thin films: materials science and basic research, volume 148. Springer Berlin, 1999.

[160] A. Triolo, O. Russina, R. Caminiti, H. Shirota, H. Y. Lee, C. S. Santos, N. S. Murthy, and J. Edward W. Castner. Comparing intermediate range order for alkyl- vs. ether-substituted cations in ionic liquids. Chemical Communications, 48(41):4959-4961, 2012.

[161] A. Triolo, O. Russina, C. Hardacre, M. Nieuwenhuyzen, M. A. Gonzalez, and H. Grimm. Relaxation processes in room temperature ionic liquids: the case of 1-butyl-3-methyl imidazolium hexafluorophosphate. The Journal of Physical Chemistry B, 109(46):22061-22066, 2005.

[162] M. Trulsson, J. Algotsson, J. Forsman, and C. E. Woodward. Differential capacitance of room temperature ionic liquids: The role of dispersion forces. The Journal of Physical Chemistry Letters, 1(8):1191-1195, 2010.

[163] D. A. Turton, J. Hunger, A. Stoppa, G. Hefter, A. Thoman, M. Walther, R. Buchner, and K. Wynne. Dynamics of imidazolium ionic liquids from a combined dielectric relaxation and optical kerr effect study: Evidence for mesoscopic aggregation. Journal of the American Chemical Society, 131(31):11140-11146, 2009.

[164] A. Uysal, H. Zhou, G. Feng, S. S. Lee, S. Li, P. Fenter, P. T. Cummings, P. F. Fulvio, S. Dai, J. K. McDonough, and Y. Gogotsi. Structural origins of potential dependent hysteresis at the electrified Graphene/Ionic liquid interface. The Journal of Physical Chemistry C, 118(1):569-574, 2014. 
[165] J. Vatamanu, O. Borodin, and G. D. Smith. Molecular insights into the potential and temperature dependences of the differential capacitance of a room-temperature ionic liquid at graphite electrodes. J. Am. Chem. Soc., 132(42):14825-14833, 2010.

[166] J. Vatamanu, O. Borodin, and G. D. Smith. Molecular simulations of the electric double layer structure, differential capacitance, and charging kinetics for $n$ methyl-n-propylpyrrolidinium bis(fluorosulfonyl)imide at graphite electrodes. $J$. Phys. Chem. B, 115(12):3073-3084, 2011.

[167] P. Walden. Molecular magnitude and electrical conductivity of some fused salts. Bulletin de l'Academie Imperiale des Sciences de St. Petersbourg, (6):405-422, 1914.

[168] T. Waldmann, H.-H. Huang, H. E. Hoster, O. Höfft, F. Endres, and R. J. Behm. Imaging an ionic liquid adlayer by scanning tunneling microscopy at the solid|vacuum interface. ChemPhysChem, 12(14):2565-2567, 2011.

[169] Y. Wang, C.-N. Sun, F. Fan, J. R. Sangoro, M. B. Berman, S. G. Greenbaum, T. A. Zawodzinski, and A. P. Sokolov. Examination of methods to determine free-ion diffusivity and number density from analysis of electrode polarization. Physical Review E, 87(4):042308, 2013.

[170] P. Wasserscheid and T. Welton. Ionic liquids in synthesis. Wiley-VCH, Weinheim, 2003.

[171] J. S. Wilkes. A short history of ionic liquids-from molten salts to neoteric solvents. Green Chemistry, 4(2):73-80, 2002.

[172] J. S. Wilkes and M. J. Zaworotko. Air and water stable 1-ethyl-3methylimidazolium based ionic liquids. Journal of the Chemical Society, Chemical Communications, (13):965-967, 1992.

[173] D. Xiao, L. G. Hines, S. Li, R. A. Bartsch, E. L. Quitevis, O. Russina, and A. Triolo. Effect of cation symmetry and alkyl chain length on the structure and intermolecular dynamics of 1,3-dialkylimidazolium bis(trifluoromethanesulfonyl)amide ionic liquids. The Journal of Physical Chemistry B, 113(18):6426-6433, 2009.

[174] K. Yamamoto, M. Tani, and M. Hangyo. Terahertz time-domain spectroscopy of imidazolium ionic liquids $\dagger$. The Journal of Physical Chemistry B, 111(18):48544859, 2007.

[175] R. Yamamoto, H. Morisaki, O. Sakata, H. Shimotani, H. Yuan, Y. Iwasa, T. Kimura, and Y. Wakabayashi. External electric field dependence of the structure of the electric double layer at an ionic liquid/Au interface. Applied Physics Letters, 101(5):053122-053122-4, 2012. 
[176] X. Zhang, Y.-X. Zhong, J.-W. Yan, Y.-Z. Su, M. Zhang, and B.-W. Mao. Probing double layer structures of au (111)-BMIPF6 ionic liquid interfaces from potential-dependent AFM force curves. Chemical Communications, 48(4):582, 2012.

[177] H. Zhao. Diffuse-charge dynamics of ionic liquids in electrochemical systems. Physical Review E, 84(5):051504, 2011.

[178] H. Zhou, M. Rouha, G. Feng, S. S. Lee, H. Docherty, P. Fenter, P. T. Cummings, P. F. Fulvio, S. Dai, J. McDonough, V. Presser, and Y. Gogotsi. Nanoscale perturbations of room temperature ionic liquid structure at charged and uncharged interfaces. ACS Nano, 6(11):9818-9827, 2012. 


\section{Danksagung}

[In der elektronischen Fassung aus Datenschutzgründen entfernt] 